Aus dem Institut für Ethik und Geschichte der Medizin Prof. Dr. C. Wiesemann der Medizinischen Fakultät der Universität Göttingen

\title{
Autonomie zwischen Ideal und Realität
}

Eine empirisch - ethische Reflexion eines Prinzips anhand ärztlicher Kommunikationsstrategien am Lebensende

\author{
INAUGURAL - DISSERTATION \\ zur Erlangung des Doktorgrades \\ der Georg-August-Universität zu Göttingen
}

\author{
vorgelegt von \\ Lena Woydack \\ aus Heide
}

Göttingen 2016 
Dekan:

I. Berichterstatterin:

II. Berichterstatter:

III. Berichterstatter:

Datum der mündlichen Prüfung:
Prof. Dr. rer. nat. H. K. Kroemer

Prof. Dr. rer. nat. S. Schicktanz (Betreuerin)

Prof. Dr. J. Schildmann (Koreferent)

Prof. Dr. T. Meyer (Promotor-Vertretung)

12.10.2017 
$\begin{array}{lr}\text { 1. Einleitung } & 1\end{array}$

2. Fragestellung 3

$\begin{array}{ll}\text { I. Binnensicht der Palliativmedizin } & 6\end{array}$

3. Der Fachbereich Palliativmedizin $\quad 6$

$\begin{array}{ll}\text { 3.1 Die Entwicklung des Fachbereichs } & 6\end{array}$

3.2 Das Selbstbild der Palliativmedizin $\quad 7$

3.3 Das therapeutische Konzept der Palliativmedizin 9

3.4 Stand der Forschung 11

3.5 Der Einfluss des Konzepts auf hierarchische Strukturen 17

$\begin{array}{lr}\text { Il. Medizinethische Perspektive } & 18\end{array}$

$\begin{array}{lr}\text { 4. Autonomie } & 18\end{array}$

4.1 Autonomie bei Kant und Mill 18

4.2 Das Prinzip der Autonomie im Diskurs der angewandten Bioethik 21

4.3 Die Prinzipienethik von Beauchamp und Childress 23

4.4 Feinbergs mehrschichtige Herangehensweise an Autonomie 26

4.5 Autonomie aus relational feministischer Perspektive 28

4.6 Gestützte Selbstbestimmung 30

4.7 Personale Autonomie 32

5. Der Begriff der Würde 35

5.1 Die kantische Herangehensweise an die Würde des Menschen 35

5.2 Würde als erworbenes Merkmal 36

5.3 Würdevolles Sterben im Rahmen der Rhetorik am Lebensende 37

5.4 Ansätze zur Problematik des Begriffs 39

6. Die Arzt-Patienten-Beziehung 43

6.1 Die Entwicklung der ärztlichen Identität 43

6.2 Das medizinische Aufklärungsgespräch $\quad 45$ 
6.3 Der informed consent als kommunikatives Gleichgewicht 49

6.4 Patientenverfügungen als kommunikatives Werkzeug 50

6.5 Die Expertenautorität des Arztes

$\begin{array}{ll}\text { 7. Zwischenfazit } & 57\end{array}$

$\begin{array}{ll}\text { 8. Methoden } & 61\end{array}$

8.1 Empirisch-qualitative Forschung anhand von Experteninterviews 61

8.2 Erstellung und orientierender Inhalt des Interviewleitfadens 63

8.3 Auswahl des Samples und Durchführung der Interviewstudie 63

8.4 Datenerfassung und Transkription $\quad 65$

8.5 Datenstrukturierung und Datenanalyse $\quad 66$

$\begin{array}{ll}\text { III. Auswertungen } & 72\end{array}$

$\begin{array}{ll}\text { 9. Ergebnisse } & 72\end{array}$

9.1 Die Grenzen des Autonomieprinzips in der medizinischen Praxis 72

9.1.1 Autonomie als anerkanntes Ideal 73

9.1.2 Spannung zwischen Theorie und Praxis im Umgang mit Autonomie 73

9.1.3 Medizinische Indikation als Grenze von Patientenautonomie 74

9.1.4 Imperativ der Eigenverantwortung des Arztes $\quad 76$

9.2 Autonomie in Wechselwirkung zu professionsspezifischen Handlungslogiken 79

9.2.1 Verflechtung von Autonomie und Kommunikation als Instrument 79

9.2.1.1 Das Idealbild des Arzt-Patienten-Gesprächs 79

9.2.1.2 Subtext des Patienten als Variable $\quad 80$

9.2.1.3 Offenheit als Grundstrategie $\quad 81$

9.2.1.4 Schlechte Erfahrung mit Offenheit 82

9.2.1.5 Problematische Konzepte $\quad 85$

9.2.2 Einschränkung von Autonomie durch Hemmungen vor dem Tod 87

9.2.3 Normierungsgefahr durch Schablonen für das Sterben 89

9.2.4 Rollenkonsistenz der einzelnen Professionen 92

9.3 Professionsimmanente Spannungen in der medizinischen Praxis 97 
$\begin{array}{ll}\text { 10. Diskussion } & 102\end{array}$

$\begin{array}{lr}\text { 11. Fazit } & 106\end{array}$

$\begin{array}{lr}\text { 12. Zusammenfassung } & 108\end{array}$

$\begin{array}{ll}\text { 13. Literaturverzeichnis } & 111\end{array}$

14. Anhang $\quad 122$

$\begin{array}{lr}\text { 15. Publikationsliste } & 124\end{array}$

$\begin{array}{lr}\text { 16. Danksagung } & 125\end{array}$ 


\section{Einleitung}

„Neben konzeptionellen Unbestimmtheiten steht also vor allem die Realitätstauglichkeit des Prinzips der Patientenautonomie in Frage. In vieler Hinsicht ist sie nicht nur ein Ideal, sondern eben auch eine Fiktion." (Feuerstein 2013, S. 249)

Die Autonomie des Patienten'1, verstanden als eine Form von Entscheidungsfreiheit und Handlungsfähigkeit, stellt aktuell den zentralen Bezugspunkt des Argumentierens in der Medizinethik dar. Das dazu zählende Recht, selbst über den eigenen Körper zu entscheiden, spielt dabei in der medizinischen Praxis und bei Fragen, die das Lebensende des Menschen betreffen, eine entscheidende Rolle. Autonomie ist indes sowohl positiv assoziierter Mittelpunkt als auch Konfliktpunkt der aktuell vorherrschenden Diskurse der Medizinethik. Beispielhaft zeigt sich dieser Aspekt anhand des Diskurses zum Umgang mit neuen Reproduktionstechniken oder anhand des Argumentationsfokus bezüglich einer möglichen Legitimation für bestimmte Formen der Sterbehilfe. In Hinblick auf die medizinische Praxis existiert zudem kaum ein vergleichbares Prinzip, das einen ähnlich prägenden Einfluss auf die Arzt-Patienten-Interaktion aufweist. Dies gilt sowohl für alltagsrelevante Entscheidungen der medizinischen Praxis als auch für die großen Kontroversen der Medizinethik. Die argumentative Fokussierung auf das Prinzip der Autonomie und die damit einhergehende Wahrnehmung des Prinzips als eine Art Korrektiv ruft jedoch auch Kritik hervor. So zeigt das einleitend aufgeführte Zitat eindrücklich die Zweifel, die mit der Reflexion über die potentielle Autonomie des Patienten einhergehen können. Über die Frage nach der Realitätstauglichkeit des Prinzips der Patientenautonomie hinaus stellt Feuerstein gar die These auf, Autonomie sei lediglich Fiktion (ebd.).

Anhand des Zitats, das sich in inhaltlich vergleichbarer Form auch in den Arbeiten anderer Autoren wiederfindet, wird somit annähernd deutlich, wie fern sich theoretischer Begriff und Praxisgeschehen zu sein scheinen.

Kritische Stimmen entwickelten sich jedoch auch innerhalb des theoretischen Diskurses selbst. Dem Autonomiediskurs wird dabei meist ein überzogener Individualismus vorgeworfen, der der sozialen Natur der Menschen und der vielfältigen sozialen Abhängigkeit von Patienten nicht gerecht wird. Ein weiterer Kernpunkt ist die Kritik an einer universal gültigen Auslegung des Autonomieprinzips (vgl. Wiesemann 2012). Die

\footnotetext{
${ }^{1}$ Im weiteren Text werden aus Gründen der besseren Lesbarkeit die Ausdrücke: Patient, Akteur, Forscher bzw. Arzt verwandt, wobei damit immer - wenn nicht ausdrücklich angegeben - beide Geschlechter gemeint sind.
} 
damit einhergehenden Ansätze fragen sämtlich nach einer situations- und problembezogenen Betrachtung des Autonomiebegriffs, die über die traditionellen Perspektiven, wie sie bei Kant (vgl. Kant 2007), Mill (vgl. Mill 1974) und Beauchamp und Childress (vgl. Beauchamp und Childress 1979) zu finden sind, hinausgehen. Diese kritischen Ansätze lassen sich unter dem Begriff der relationalen Autonomie zusammenfassen.

Insgesamt hat die Komplexität medizinethischer Probleme wie auch der stets politische und kulturelle Charakter medizinethischer Kontroversen somit dazu geführt, dass sich das einst klare Profil des Autonomieprinzips in sehr unterschiedlichen Diskurslinien weiterentwickelt hat. Trotz dieser ausdifferenzierten Weiterentwicklung scheint jedoch eine letzte Ebene der Reflexion zu fehlen. Formulieren die Akteure des medizinischen Praxisgeschehens, Autonomie fungiere lediglich als ein fiktiver Begriff, so scheint offenkundig eine Verzahnung von theoretischem Diskurs und medizinischer Praxis zu fehlen. Und dies trotz des bereits existierenden Diskurses zur relationalen Autonomie.

Das einleitende Zitat deutet vorsichtig an, dass dieser Aspekt besonders in Bezug auf schwierige Situationen entscheidend ist. So kann beispielsweise der biographische Bruch durch eine zum Tode führende Erkrankung verantwortlich dafür sein, dass bisher bestehende Vorstellungen und Prinzipien in den Hintergrund rücken. Wie zahlreiche Beispiele aus der Praxis zeigen, ist es demnach nicht ausreichend, das Prinzip der Autonomie im Sinne eines universal gültigen Prinzips seinen Handlungen und Entscheidungen theoretisch zugrunde zu legen. Es bedarf stets der konkreten Frage nach der Realisierbarkeit eines derartigen Prinzips und der Frage nach seiner Daseinsberechtigung als eine Art Korrektiv für das menschliche Handeln und Entscheiden bei Fragen am Lebensende. 


\section{Fragestellung}

Das vorliegende Forschungsvorhaben widmet sich der Frage der Realisierbarkeit des Autonomieprinzips in der medizinischen Praxis. Hintergrund dafür bilden die differenzierten theoretischen Arbeiten zum Autonomiebegriff in der Medizinethik. Die Analyse soll dabei nicht primär mit einem weiteren theoretischen Kritikpunkt einhergehen, sondern einen konstruktiven Blick in die Praxis einbringen.

Neben dem Abgleich mit dem Autonomiediskurs, wie er sich in der Medizinethik vollzogen hat, soll das Autonomieprinzip, wie es als Ideal in der Palliativmedizin theoretisch entworfen wird, wesentliche Analysegrundlage sein. Im Rahmen dessen soll herausgearbeitet werden, wie sich das Prinzip der Autonomie aus der Perspektive von Palliativmedizinern und kurativ tätigen Medizinern in deren Handlungswirklichkeit darstellt. Die Palliativmedizin als ein verhältnismäßig junger Fachbereich des bestehenden Medizinsystems hat in ihren vielschichtigen Idealen das Prinzip der Autonomie als eines der zentralen Zielsetzungen formuliert und festgelegt. Die Palliativmedizin, die als einziges Fach ethische Fragestellung in seine Konzepte integriert und im Rahmen dessen die Autonomie des Patienten stark macht, hat somit eine Pionierfunktion im aktuellen Medizinsystem inne.

Außerdem ist die Medizin mit der Etablierung des Fachbereichs und seinem Fokus auf Selbstbestimmung am Lebensende damit einer öffentlich formulierten Kritik gegenüber Ärzten konstruktiv entgegengetreten. Der Kritikpunkt, der auch seitens einiger Bioethiker, unter anderem Maio, formuliert wurde, geht davon aus, dass Ärzte im Zuge der (Hochtechnologie-) Medizin zunehmend den Tod nicht mehr akzeptieren können und damit die Selbstbestimmung bei Fragen am Lebensende zu wenig Berücksichtigung fände (vgl. Maio 2013). Es scheint also innerhalb des Medizinsystems durch die Entwicklung der Palliativmedizin Lösungsansätze zu geben, die einen offeneren Umgang mit dem Thema Sterben und Tod ermöglichen und innerhalb der Arzt-Patienten-Beziehung weitere Ebenen für Autonomie eröffnen. Die Ideale der Palliativmedizin können in diesem Sinne als eine Art Korrektiv für das medizinische Praxisgeschehen betrachtet werden, welches sowohl kurativ als auch palliativ tätige Ärzte dahingehend sensibilisieren soll, mehr Raum für den Subtext der (Arzt -) Patienten - Kommunikation zu ermöglichen und jenseits des informed consent nach Patientenautonomie zu fragen. Da sich theoretischer Diskurs und komplexes Praxisgeschehen jedoch häufig wenig berühren, soll insbesondere untersucht werden, inwiefern das theoretische Konzept der Autonomie in der Palliativmedizin für die Praxis handlungsrelevant wird und wie sich im Umgang damit alternative Überlegungen der 
medizinethischen Debatte als hilfreich erweisen können. Das Kernanliegen des vorliegenden Forschungsvorhabens ist dabei die Frage nach der generellen Realisierbarkeit des Autonomieprinzips in der medizinischen Praxis. Darüber hinaus soll eine Reflexion über die Legitimation des Autonomieprinzips im Sinne eines allgemein gültigen Korrektivs erfolgen. Dies ist insbesondere mit Blick auf die in der medizinischen Praxis sehr unterschiedlichen Patienten und eine sich insgesamt pluralisierende Gesellschaft zwingend notwendig. Da Ideale wie die Förderung von Patientenautonomie stets eine präskriptive Dimension haben, soll zudem diskutiert werden, ob die Etablierung einer bestimmten Idealvorstellung, insbesondere bei Fragen am Lebensende, überhaupt handlungsrelevant werden kann und wie man sie letztlich explizit machen kann. Für Patienten, die ihr Leben in unterschiedlichen biographischen Strukturen leben und bezüglich des eigenen Lebens gewisse normative Erwartungen internalisiert haben, kann dies mit dem eigentlich individuellen Umgang mit dem Tod eine erhebliche Konsequenz haben. Ein weiterer Gedankenansatz, der im Verlauf des Forschungsprozesses entstanden ist und im Verlauf diskutiert wird, bringt die Überlegung mit sich, inwiefern die Bedeutung von Rollenbildern in die Erwägung zur Realisierbarkeit des Autonomieprinzips eine Rolle spielen.

Für die vorliegenden Untersuchungen wurde die sozialwissenschaftliche Methodik der qualitativen Interviewstudie gewählt. Im Konkreten bildet das Leitfaden-gestützte Experteninterview den methodischen Hintergrund des vorliegenden Forschungsvorhabens. Seine Bedeutung findet das Experteninterview in der Rekonstruktion komplexer Wissensbestände, die für die Erklärung sozialer Phänomene relevant sind (siehe Kapitel 8.).

Die vorliegende Arbeit verknüpft über ihren methodischen und ihren theoretischen Hintergrund also einen ethischen und einen operationalen Zugang. Die Intention, eine Verzahnung zwischen Theorie und Praxis herzustellen, wird dadurch gewährleistet. Darüber hinaus werden normative Elemente der Medizinethik und deskriptive Elemente empirischer Forschung miteinander verknüpft.

In Hinblick auf die Interaktion von Arzt und Patient werden die genannten Aspekte bei Fragen am Lebensende und bei Erkrankungen mit einer infausten Prognose besonders greifbar. Aus diesem Grund beschränken sich die Analysen auf diesen Rahmen und legen, wie beschrieben, einen Fokus auf die Ideale des Fachbereichs Palliativmedizin. Da das formulierte Forschungsanliegen für alle praktizierenden Mediziner von Relevanz ist, die mit sterbenden Patienten arbeiten, wurden trotz des genannten Fokus jedoch nicht ausschließlich Palliativmediziner in die Studie aufgenommen. Vergleichend wurde im 
Rahmen der Untersuchungen eine heterogene Gruppe praktizierender Mediziner befragt, die in häufigem Kontakt zu Patienten mit infausten Prognosen oder zu Sterbenden stehen. Die Analyse soll in erster Linie anhand der erzählten Wahrnehmung von Medizinern erfolgen. Dabei werden die Wahrnehmungen von Ärzten hinsichtlich ihrer Kommunikationsstrategien und der Einfluss auf die Autonomie des Patienten untersucht. In dem Analyseansatz wird Kommunikation explizit als Instrument betrachtet, das prinzipiell für sich stehend bereits Autonomie-fördernd ist. Zudem soll beleuchtet werden, ob eine Überbetonung von Autonomie dazu führen kann, dass Konflikte entstehen oder dass Handlungsspielräume von Patienten potentiell eingeschränkt werden. Bezogen auf die Bedeutung des Autonomiebegriffs für die Praxis schränken die vorliegenden Überlegungen dessen Bedeutung nicht ein, sondern es soll vielmehr verdeutlicht werden, wie notwendig eine Auseinandersetzung mit dem Begriff und seinem Prinzipiencharakter für eine reflektierte Praxis ist. 


\section{Binnensicht der Palliativmedizin}

\section{Der Fachbereich Palliativmedizin}

\subsection{Die Entwicklung des Fachbereichs}

Inspiriert durch die Pionierin Cicley Saunders, die 1967 in England das St Christopher's Hospice gründete, entstand in Deutschland 1986 das erste stationäre Hospiz und 1983 die erste Palliativstation an der Universitätsklinik Köln (Kettler 2006). Die Hospizbewegung und die Palliativmedizin sind jedoch trotz gleicher Wurzeln in Deutschland und einer zeitlich parallelen Entwicklung unterschiedliche Wege gegangen. Während Hospize als eigenständige Einrichtungen entstanden, in denen die ganzheitliche Betreuung Sterbender im Zentrum der Bemühungen steht, hat sich die Palliativmedizin in Deutschland als Handlungsbereich von Ärzten innerhalb des medizinischen Systems etabliert.

Die rasante Entwicklung, die sich in den vergangenen Jahren in der Palliativmedizin vollzogen hat, zeigt sich unter anderem an den zahlreichen neu errichteten palliativmedizinischen Einrichtungen. Während im Jahr 2000 bereits 62 Palliativstationen existierten, von denen fünf an Universitätskliniken angegliedert waren (Sabatowski 2001), bestehen im Jahr 2015 bundesweit 289 Stationen und 237 Teams der Spezialisierten ambulanten Palliativversorgung (Deutsche Gesellschaft für Palliativmedizin 2015). Neben der Zunahme palliativmedizinscher stationärer Einrichtungen wird der Fortschritt des Fachbereichs anhand einer Intensivierung der Forschung und der Einführung einheitlicher Dokumentations- und Qualitätssicherungssysteme deutlich. Zudem ist der Fachbereich seit dem Jahr 2012 fest als Pflichtlehrfach in der universitären Lehre für Studierende der Medizin verankert.

Ein interessanter Aspekt ist jedoch, dass die Palliativmedizin als eine neue Institution innerhalb des bestehenden Medizinsystems bis heute eine Sonderrolle innehat. Beispielsweise deutlich wird diese Sonderstellung anhand der Tatsache, dass mit Ausnahme der Universitätsklinik Köln alle palliativmedzinischen Professuren bisher noch Stiftunglehrstühle sind und dass die wichtigsten Gesetzgebungen zur Finanzierung, wie beispielsweise das Gesetz zur Spezialisierten Ambulanten Palliativversorgung erst im Jahr 2013 verabschiedet wurden.

Grundsätzlich ist es unstrittig, dass die Palliativmedizin Kernelement einer sehr bereichernden Dynamik ist. In seinen vielfältigen Idealen hat der Fachbereich das Prinzip der Autonomie als eines der zentralen Werte formuliert und festgelegt. Die Palliativmedizin 
unterstützt somit den aktuell zentralen Bezugspunkt des Argumentierens der Medizinethik und übernimmt dadurch eine Art Vorreiterfunktion im bestehenden Medizinsystem. Mit diesem Ziel und dem Bestreben, einen offeneren Umgang mit dem Sterben zu realisieren, wurde zudem eine gesellschaftliche Debatte und eine bestehende öffentliche Kritik am Medizinsystem aufgenommen und thematisiert. Auf diese wird im Verlauf der vorliegenden Arbeit detailliert eingegangen. Trotz der eindeutig positiv assoziierten Dynamik soll im Rahmen des Forschungsvorhabens genauer auf die initialen Beweggründe für die Entstehung des Fachbereichs Palliativmedizin geblickt werden. Mit Hilfe dieser Überlegung soll der Zugang zu potentiellen Schwierigkeiten und Spannungen erleichtert werden, welche mit der Etablierung einer ganzheitlichen und offeneren Arzt-PatientenInteraktion am Lebensende einhergehen können.

\subsection{Das Selbstbild der Palliativmedizin}

Palliativmedizin ist die Behandlung von Patienten mit einer nicht heilbaren progredienten und weit fortgeschrittenen Erkrankung mit begrenzter Lebenserwartung, für die das Hauptziel der Begleitung die Lebensqualität ist. Palliativmedizin soll sich dabei nicht auf die letzte Lebensphase beschränken. Viele Grundsätze der Palliativmedizin sind auch in frühen Krankheitsstadien zusammen mit der kausalen Therapie anwendbar. Palliative Zielsetzungen können in verschiedenen organisatorischen Rahmen sowohl im ambulanten wie im stationären Bereich verfolgt werden. (Deutsche Gesellschaft für Palliativmedizin 2015)

Darüber hinaus versteht die Palliativmedizin in Deutschland ihren Auftrag vor allem als Krisenintervention im Rahmen der Behandlung von Patienten mit unheilbaren Krankheiten. Es ist stets Ziel Patienten in die Eigenversorgung oder andere unterstützende Einrichtungen, wie stationäre Hospitze, zu entlassen und sie dabei fortlaufend zu unterstützen. Die Palliativmedizin sieht sich außerdem als eine Medizin, die auch nicht-kuratives Handeln als zentrale Aufgabe medizinischer Ausbildung und ärztlicher Praxis begreift. Sie nimmt damit Abstand von der Vorstellung jeden Patienten um jeden Preis heilen zu wollen. Aufgabenbereich des Fachbereichs Palliativmedizin ist es, Patienten durch symptombezogene Schmerzbehandlung, psychologische Unterstützung und menschliche Zuwendung möglichst lange in seiner freien Selbstbestimmung zu stärken und diese zu erhalten. Die Autonomie des Patienten zu stärken bedeutet hier, nicht nur die Entscheidung des Patienten im Sinne des informed consent zu respektieren, 
sondern die Autonomie des Patienten selbst zu einem Ziel palliativer Behandlung zu machen (vgl. Klaschik et al. 2001). Grundlage für die Entscheidung für oder gegen die Begrenzung einer medizinischen Maßnahme soll dabei ein Gleichgewicht zwischen dem autonomen Willen des Patienten und der medizinischen Indikation sein.

In der palliativmedizinischen Grundlagenliteratur wird dabei kein expliziter Autonomiebgriff definiert. Die Verwendung des Begriffs legt jedoch nahe, dass dieser im Sinne einer Form von Entscheidungsfreiheit und Handlungsfähigkeit in Bezug auf den eigenen Körper gelesen wird.

Im Rahmen ihres Selbstverständnisses distanziert sich die Palliativmedizin in Deutschland eindeutig von jedweder Form der Sterbehilfe. Im Zuge dessen versteht sie sich sogar als mögliche Alternative, wie es beispielsweise in einer Studie von Beckmann diskutiert wird. Beckmann betont im Rahmen seiner Arbeit, dass die Palliativmedizin in ihrem Wirken als mögliche Antwort auf die aktuellen Fragen der Sterbehilfedebatte dienen kann (vgl. Beckmann 2002). Mit Hilfe der Stärkung der Selbstbestimmungsfähigkeit des Patienten und der Etablierung eines bewussteren Umgangs mit Problemen und Ängsten des Sterbenden kann ein autonomes Entscheiden in der letzten Lebensphase gewährleistet und der Wunsch nach ärztlich assistiertem Suizid in den Hintergrund gerückt werden.

Palliation intendiert in ihrer Zielsetzung, nämlich Hilfe zur Erhaltung der Freiheit der Selbstbestimmung des Menschen, weder direkt noch indirekt den Tod des Patienten, sie ist mithin nicht Mittel zum Tod, sondern Mittel zur bewussten Annahme menschlicher Endlichkeit. (Beckmann 2002, S. 118).

Nach Student und Napiwotzky prägen die folgenden Kennzeichen die Arbeit einer palliativmedizinischen Einrichtung (Student und Napiwotzky 2007, S.4-12): Der sterbende Mensch und seine Angehörigen stehen im Zentrum der Bemühung. Die Kontrolle über die Situation liegt also bei den Betroffenen. Ihnen steht ein interdisziplinär agierendes und multiprofessionelles Team zur Verfügung, welches nicht nur aus medizinischem Personal besteht. Die Teammitglieder verfügen über gute Kenntnisse in der Symptomkontrolle und die Ideen Ehrenamtlicher werden stark mit einbezogen. Hierbei geht es nicht nur um medikamentöse Strategien, sondern ebenso um die übrigen „Dimensionen menschlicher Existenz" (ebd. S.4-12). Außerdem zählt nach Student und Napiwotzky die Kontinuität der Unterstützung des Betroffenen zu den Merkmalen einer umfassenden palliativen Betreuung. Ein weiteres Ziel der Palliativmedizin ist es, dem Patienten mehr Normalität im Rahmen seiner Erkrankung zu ermöglichen. Indem die Erkrankung stärker in den Alltag des Patienten eingebunden wird, eröffnet sich zwischen Arzt und Patient eine zusätzliche 
Ebene, auf der Ärzte ihre Patienten wahrnehmen und ernst nehmen müssen. Um dies zu realisieren spielen neben einem kompetenten und interdisziplinär agierendem Team Fähigkeiten, wie Empathie und eine gute Kommunikationsfähigkeit eine wichtige Rolle (ebd.).

Einen weiteren Ansatz zur Definition der Säulen der palliativmedizinischen Betreuung findet man bei Becker und Xander. Sie betonen neben den medizinischen Behandlungsstrategien ein multiprofessionell organisiertes Gesamtkonzept mit dem Ziel, dem Patienten in seiner verbleibenden Zeit ein möglichst hohes Maß an Lebensqualität zu ermöglichen (vgl. Becker und Xander 2008). Die Autorinnen führen in diesem Zusammenhang explizit die Bereiche Symptomkontrolle, Rehabilitation, Aspekte der Kommunikation und Patientenbetreuung in der Terminalphase an (ebd.). Hinsichtlich konkreter Kommunikationsformen stützen sich die Autorinnen auf das klassische Modell der partizipativen Entscheidungsfindung und betonen an dieser Stelle den besonderen Wert des Modells im palliativmedizinischen Kontext als eine „Alternative zur Fokussierung auf den Autonomierespekt" (ebd., S.56). Das Vorgehen, gemeinsam eine Therapieentscheidung auszuhandeln verlangt, dass Ärzte gemeinsam mit ihren Patienten immer wieder auf verschiedenen Ebenen in einen Prozess der Entscheidungsfindung eintreten (ebd.). Die Autorinnen betonen in diesem Zuge den Wert von Kommunikationsprozessen für die Förderung von Patientenautonomie. In ihren Ausarbeitungen wird jedoch auch ein innerer Konflikt deutlich. Die Palliativmedizin fokussiert mit ihren Idealen die Förderung von Patientenautonomie und die damit einhergehenden offenen Kommunikationsprozesse, wirt jedoch innerhalb eigener Arbeiten die Überlegung auf, dass genau in Bezug auf diesen Aspekt in bestimmten Situationen Alternativen benötigt werden.

So wie es auch Becker und Xander diskutieren, scheinen demnach im konkreten Praxisgeschehen Alternativen zu dem Fokus auf das Autonomieprinzip zu fehlen (vgl. ebd.). Analog zu der Forschungsintention der vorliegenden Arbeit soll auf genau diesen Aspekt im Verlauf näher eingegangen werden.

\subsection{Das therapeutische Konzept der Palliativmedizin}

Betrachtet man den begrifflichen Ursprung der Palliativmedizin, der sich von pallium, der Mantel, und palliare, lindern, ableiten lässt, bekommt man eine Vorstellung für das medizinische, aber auch für das im vorangegangenen Kapitel beschriebene Gesamtkonzept des Fachbereichs. 
Mit Hilfe eines ganzheitlichen und multiprofessionellen Therapieansatzes soll Patienten mit einer infausten Prognose die letzte Lebenszeit erleichtert und verbessert werden. Vor dem Hintergrund des namengebenden Begriffes geschieht dies, indem die Patienten die verbleibende Lebenszeit durch einen schützenden Mantel abgeschirmt von belastenden Symptomen ihrer Erkrankung durch ihr Leben begleitet werden sollen (AichmüllerLietzmann 1998).

Der multiprofessionelle Ansatz bedeutet eine fächerübergreifende Zusammenarbeit zwischen Ärzten unterschiedlicher Fachdisziplinen und anderer Berufsgruppen, wie beispielsweise Pflegepersonal, Psychologen und Seelsorger. Zur Ganzheitlichkeit gehören immer auch die Beachtung von psychischen, sozialen und spirituellen Anliegen, sowie Problemen. Somit werden unter Berücksichtigung verschiedener Einflussfaktoren, zum Beispiel auf das Schmerzerleben, Symptome innerhalb eines palliativmedizinischen Gesamtkonzeptes therapiert (vgl. Beck und Kettler 2001). In die medizinische Behandlung werden neben medikamentösen und nicht medikamentösen auch strahlentherapeutische und chirurgische Interventionen zur Symptomlinderung integriert. Der Tod soll dabei weder hinausgezögert noch beschleunigt werden. Palliativmedizin soll nach Klaschik stets als eine „bejahende Lebenshilfe“ verstanden werden (Klaschik et al. 2001, S.71). Konkrete Behandlungsansätze umfassen die Linderung krankheitsassoziierter, meist tumorassoziierter Symptome, wie beispielsweise Schmerzen, Luftnot, Übelkeit und Erbrechen. Die hier erwähnten stellen die häufigsten zu behandelnden Symptome in der palliativmedizinischen Betreuung dar und können zu einer erheblichen Einschränkung der Lebensqualität führen (Radbruch et al. 2003).

Was die Organisationsstrukturen anbelangt existiert neben der stationären Behandlung eine umfassende Betreuungsmöglichkeit durch die Spezialisierte Ambulante Palliativversorgung (SAPV). „Diese beinhaltet insbesondere spezialisierte palliativärztliche und palliativpflegerische Beratung und/oder (Teil-)Versorgung, einschließlich der Koordination von notwendigen Versorgungsleistungen bis hin zu einem umfassenden, individuellen Unterstützungsmanagement.“ (DGP 2013). Außerdem wird Patienten durch das Netzwerk der SAPV eine 24-stündige Erreichbarkeit gewährleistet. Die Teams arbeiten in enger Zusammenarbeit mit niedergelassenen Ärzten, Pflegediensten, Krankenhäusern und stationären Pflegeeinrichtungen und führen regelmäßige Teamsitzungen und Fallbesprechungen durch. So kann das Netzwerk der Spezialisierten Ambulanten Palliativversorgung als zusätzliche und auch alleinige Unterstützung zur Rate gezogen werden, sowie auch als beratende und koordinierende Instanz. 


\subsection{Stand der Forschung}

In Deutschland sind die sozialwissenschaftlichen Publikationen auf dem Gebiet der Palliativmedizin alle noch sehr jung und bewegen sich methodisch bisher eher in dem Rahmen quantitativer Studien. Im folgenden soll eine Auswahl der palliativmedizinischen Forschungsarbeiten und der daraus hervorgegangenen Daten aus den vergangenen Jahren vorgestellt werden.

Stiehl et al. untersuchten mit Hilfe der Hospitz- und Palliativerhebung (HOPE) die quantitative Entwicklung der Palliativmedizin. Die Studie lief in dem Zeitraum von 2004 bis 2009 und kam abschließend zu dem Ergebnis, dass sich zunehmend mehr Patienten mit anderen Diagnosen als Tumordiagnosen in palliativmedizinischer Behandlung befinden. Dieser Aspekt kann als Zeichen dafür gedeutet werden, dass die Notwendigkeit und die Effizienz von palliativmedizinischen Behandlungen für diese Patienten auch zu anderen Fachbereichen vorgedrungen sind. Außerdem sind im Laufe der vergangenen zehn Jahre deutlich mehr palliativmedizinische Einrichtungen errichtet worden (vgl. Stiehl et al. 2010). Ein Zeichen für die zunehmende Präsenz der Palliativversorgung in der Öffentlichkeit und in medizinischen Fachbereichen ist das im November 2013 vom GKV-Spitzenverband verabschiedete Gesetz zur Spezialisierten Ambulanten Palliativversorgung (Deutsche Gesellschaft für Palliativmedizin 2015). Mit in Kraft treten des Gesetzes sind Organisation und Finanzierung deutlich klarer geregelt als in den vergangenen Jahren. Wesentlich beratend tätig war hierbei die Deutsche Gesellschaft für Palliativmedizin.

Nauck et al. beleuchteten in einer Studie mit Hilfe von Fragebögen die Organisation und die Qualität der ärztlichen Versorgung in den Hospizen in Nordrhein-Westfalen vor der Einführung der Spezialisierten ambulanten Palliativversorgung (vgl. Nauck et al. 2011). Es wurden sowohl die Hospizleiter als auch die behandelnden Ärzte befragt. Die Ergebnisse wurden interdisziplinär diskutiert und der Versuch unternommen, die Erkenntnisse in den klinischen Kontext zu integrieren. Die Studie ergab, dass sich der Großteil der ärztlichen Versorgung in den Händen der Hausärzte befindet, von denen zu dem Zeitpunkt der Befragung ca. $60 \%$ in dem Besitz einer oder mehrerer Zusatzbezeichnungen in dem Bereich Palliativmedizin waren. Von Seiten der Ärzte wurden laut der Studie die größten bemängelten Defizite im Bereich Kommunikation und Gesprächsführung sowie Spiritualität beschrieben. Die Selbstreflexion bezüglich vorhandener Defizite im Bereich Kommunikationsfähigkeit unterstützt das Forschungsvorhaben der vorliegenden Arbeit, kommunikative Fähigkeiten und rhetorische Stilmittel bei Ärzten näher zu beleuchten. Bei den Hospizleitern entstand die größte Unzufriedenheit durch eine nicht beherrschbare 
Symptomkontrolle und durch die mangelnde Akzeptanz des Sterbens auf Seiten der Ärzte. Die meisten Umfrageteilnehmer sahen eine Beratung durch einen qualifizierten Palliativmediziner als sinnvolle Ergänzung der Betreuung an. Insbesondere im Bereich von ethischen und speziellen palliativmedizinischen Fragestellungen sowie in Akutsituationen sei eine ständige Erreichbarkeit eines erfahrenen Pallitativmediziners wichtiger als dessen ständige Präsenz. Die Studie benennt zudem die Problematik, dass es trotz bestehender Bereitschaft zur Fortbildung immer noch unzureichend viele palliativmedizinisch ausgebildete Ärzte gibt. Wesentliche ethische Fragen, die im Rahmen der Studie diskutiert wurden, sind eine unklare Regelung für eine mögliche Therapiebegrenzung und die unzureichende psychosoziale Betreuung der Hospizbewohner.

Zudem wurden in jüngerer Vergangenheit Studien zum dem Thema Lehre der Palliativmedizin in Deutschland durchgeführt. Die Studien kommen darin überein, dass es prinzipiell wertvoll ist das Fach Palliativmedizin in die medizinische Ausbildung zu integrieren, im Zuge dessen sollten laut Untersuchungen jedoch auch einheitliche und strukturierte Ausbildungskonzepte entwickelt werden. Beispielsweise untersuchten Laske et al. mit Hilfe einer quantitativen Studie, die sich an alle 36 Studiendekanate, Studierende und Dozenten wandte, den Stand der Lehre in Deutschland. Laske et al. forderten bereits 2009 eine umfangreiche und professionelle Lehre für alle Sudierende der Medizin. Diese sollte im Zusammenspiel mit den vorhandenen palliativmedizinischen Infrastrukturen, z.B. in Anlehnung an das Curriculum zu den Grundlagen der Palliativmedizin für Studierende in der Medizin der Deutschen Gesellschaft für Palliativmedizin stattfinden (vgl. Laske et al. 2009).

Seit 2009 ist die Palliativmedizin offiziell Querschnittsbereich, Lehr- und Prüfungsfach an allen deutschen medizinischen Fakultäten. Während die Universitäten es bisher frei handhaben konnten, in welchem Umfang sie den Fachbereich in die Lehre integrieren, ist es erst ab dem Sommersemester 2014, Pflicht einen Leistungsnachweis für das Fach Palliativmedizin zu erwerben. Ältere Untersuchungen zeigen insgesamt noch bestehende Defizite bei einer hinreichenden Lehre im Umgang mit Sterbenden und Schwerkranken auf (vgl. Klaschik et al. 2002, A1286). Mit der Änderung des Curriculums wäre es sinnvoll, weitere Studien folgen zu lassen.

Eine qualitative Studie, aus der Ergebnisse zu dem Stand und den Problemen der Palliativmedizin beziehungsweise von Palliative Care in Deutschland publiziert wurden, lieferten Behmann et al. Als Teilelement einer großen Arbeit wurden fünf Interviews mit Experten aus dem Bereich Palliativmedizin und fünf Public Health Experten im Rahmen eines semistrukturierten Interviews geführt (vgl. Behmann 2009). Aufgrund der 
demographischen Entwicklung sprachen sich beide Gruppen dafür aus, vermehrt ältere Patienten und auch Patienten mit anderen Erkrankungen als Tumorerkrankungen in die palliativmedizinische Betreuung aufzunehmen. Abschließend Erwähnung fand die Tatsache, dass beide Expertengruppen das Thema Pädiatrische Palliativmedizin nicht in die Diskussionen aufgenommen hatten. Relevante Probleme, die Erwähnung fanden, sind die zu geringe Einbindung und Integration der Palliativmedizin in das deutsche Gesundheitssystem und die zu dem damaligen Zeitpunkt noch ungeklärte Finanzierung der Palliativmedizin in Deutschland. Ohne dies weiter auszuführen gilt es laut Aussagen der Studie als problematisch, dass es rund um den Fachbereich Palliativmedizin keine klaren Verantwortungsbereiche zu geben scheint. Dieser Aspekt könnte darauf zurückzuführen sein, dass in dem in Deutschland stark hierarchisch geprägten medizinischen System die Palliativmedizin noch keinen fest etablierten Platz eingenommen hat.

Über die Betrachtung der Lehr- und Organisationsstrukturen hinaus soll es im Folgenden um konkrete inhaltliche Aspekte gehen, die mit der Etablierung des Fachbereichs Palliativmedizin einhergehen.

Eine interessante Studie, die Berührungspunkte mit vorliegenden Forschungsanliegen aufweist, ist eine Querschnittsstudie einer palliativmedizinischen Forschungsgruppe des Universitätsklinikums Freiburg aus dem Jahr 2004. Die von Becker und Xander durchgeführte Studie fand in Zusammenarbeit mit der Ethik-Kommission der Universität statt. Im Fokus der Analysen stand der prinzipielle Umgang mit Patientenautonomie. Darüber hinaus wurden konkrete Strategien zur Förderung der Autonomie des Patienten untersucht. Im Rahmen einer quantitativen Untersuchung wurden hierfür unterschiedliche Berufsgruppen im Arbeitsfeld Palliativmedizin befragt. Im Rahmen der Ergebnisse wurde die Bedeutung von Kommunikationsprozessen besonderes betont. Die aktive Gestaltung von Kommunikation spielt zum einen bei der Förderung von Patientenautonomie und zum anderen bei gemeinsamen Entscheidungsfindungen eine bedeutende Rolle. Abschließend wird betont, dass es insbesondere im palliativmedizischen Kontext nicht ausreichend ist, den Respekt vor der Autonomie des Patienten lediglich zu fordern. Eben diese Intention muss laut Studienergebnissen stets mit einer Reflexion bezüglich der Situation des Patienten einhergehen. Die Autorinnen diskutieren diesbezüglich, dass sämtliche Patienten zusätzlich immer auch auf die Sorgepflicht des Behandlerteams angewiesen sind (vgl. Becker und Xander 2008).

Für erhebliche Diskussionen sorgte eine Studie von Schildmann et al. (vgl. Schildmann et al. 2010). Mittels eines anonymisierten Fragebogens wurden Ärzte, insbesondere 
Spezialisten aus der Palliativmedizin, zu ihren Praktiken und Entscheidungen am Lebensende befragt. Die Fragen bezogen sich auf verstorbene Patienten, die sich einer palliativen Behandlung unterzogen hatten. Es stellte sich heraus, dass entgegen des in der Palliativmedizin propagierten Standpunkts, sich klar von einem ärztlich assistierten Suizid zu distanzieren, sich circa ein Prozent der befragten Palliativmediziner für lebensverkürzende Maßnahmen entschieden hatten. Im Rahmen der Studienergebnisse wurde neben der erhobenen Kritik jedoch zur Diskussion gestellt, ob nicht gerade diese, in Einzelfällen getroffene Entscheidung, ein Zeichen für die besondere Beachtung der Selbstbestimmung des Patienten darstellen würde.

Eine weitere Studie, die auf einer anderen Ebene Aufsehen erregt hat, lieferten Temel et al. (vgl. Temel et al. 2010). Im Rahmen der quantitativen Studie wurde von Juni 2006 bis Juli 2009 in einer Klinik in Boston untersucht, inwiefern an Lungenkrebs erkrankte Patienten parallel zur klassisch onkologischen Therapie von einer zusätzlichen Palliativtherapie profitierten. Im Rahmen des Studiendesigns wurden insgesamt 151 Patienten mit der Erstdiagnose eines metastasierten Lungenkarzinoms eingeschlossen. Es gab dabei zwei Kohorten: Die erste erhielt ausschließlich eine onkologische Standardtherapie, die zweite erhielt ab dem Zeitpunkt der Diagnosenstellung zusätzlich eine unterstützende Betreuung durch ein Palliativteam. Untersucht wurde die Lebensqualität und die Stimmung beispielsweise mit Hilfe des Functional Assessment of Cancer Therapy-Lung (FACT-L) scales². Zudem wurde die Hospital Anxiety and Depression Scale ${ }^{3}$ zur weiteren Evaluation von Ängsten und Depressionen im Rahmen der Behandlung angewendet. Es zeigte sich, dass die Patienten mit palliativmedizinischer Zusatzbehandlung angaben, signifikant mehr Lebensqualität zu haben, dass sie unter weniger Depressionen litten und am Ende der Behandlung weniger aggressive Chemotherapie erhielten. Außerdem zeigte die Studie, dass die palliativmedizinisch behandelten Patienten insgesamt ca. zwei Monate länger lebten als die Patienten, die ausschließlich eine onkologische Standardtherapie erhielten. Betont wurde, dass besonderes in den ersten Behandlungsmonaten lediglich alle paar Wochen ein Kontakt zwischen den an Lungenkrebs erkrankten Patienten und dem Palliativteam bestand. Die Patienten hatten zu diesem Zeitpunkt zwar bereits ihre schwerwiegende Diagnose erhalten, waren dabei jedoch alle noch in einer körperlichen Verfassung, die keine 
intensive palliativmedizinische Behandlung notwendig machte. In Zusammenschau der Ergebnisse wird deutlich, dass der Wert, von dem die Patienten laut der Studienergebnisse profitierten, nicht primär auf medizinische Aspekte, wie die Symptomkontrolle zurückzuführen ist. Es scheint vielmehr so zu sein, dass die seitens der Patienten empfundene höhere Lebensqualität in der langfristigen, möglicherweise rein menschlichen Mitbetreuung durch das Palliativteam zu suchen ist.

Ergänzend zu der Studie von Temel et al. soll im Folgenden auf eine weitere Arbeit eingegangen werden, die sich mit dem Versuch das palliativmedizinische Behandlungskonzept in das Medizinsystem zu etablieren befasst hat. In der Studie von Herman et al. wurde die Übereinstimmung von Ärzten und Patienten verglichen, die aus ihrer jeweils eigenen Perspektive die Qualität einer palliativmedizinischen Behandlung beurteilen sollten (vgl. Hermann et al. 2010). Zum einen wurden Patienten befragt, die sich am Lebensende in einer rein hausärztlichen Behandlung befanden. Als Vergleich dazu wurden Patienten befragt, deren Hausärzte zuvor an einer palliativmedizinischen Fortbildung teilgenommen hatten. In einer Beobachtungsstudie wurde überprüft, ob die Übereinstimmung zwischen Patienten und Hausärzten, die an einer palliativmedizinischen Fortbildung teilnahmen, höher ist als bei Hausärzten ohne Fortbildung. Es zeigte sich, dass Ärzte ihre Betreuungsqualität im Vergleich zu ihren Patienten überschätzten und dass die Patienten zwischen Ärzten mit und ohne Fortbildung keine Qualitätsunterschiede bezüglich ihrer Versorgung und dem ärztlichen Umgang mit der Erkrankung wahrnahmen. Die genannte Beobachtung zeigt, dass es offensichtlich nicht möglich ist ein theoretisch wertvolles Konzept mit Hilfe einer einzigen Fortbildung in die zuvor bestehenden Arbeitsweisen und Umgangsformen eines praktizierenden Mediziners zu etablieren. Auch wenn es sich bei der Studie nur um eine Stichprobe handelt sollte auf ärztlicher Seite ganz besonders der Aspekt der Selbstüberschätzung reflektiert werden. Insbesondere hinsichtlich der Ebenen, die im Rahmen der Ideale der Palliativmedizin über die klassisch medizinischen Aspekte, wie die Symptomkontrolle, hinaus gehen.

Prinzipiell zeigt sich anhand dieser beispielhaft aufgeführten Studie, dass hinsichtlich der unterschiedlichen Bereiche des Medizinsystems eindeutig noch Forschungsbedarf besteht, inwiefern das Konzept der Palliativmedizin so umgesetzt werden kann, dass die Akteure des Praxisgeschehens von den theoretisch entwickelten Idealen profitieren können.

In Anlehnung an diese Schwierigkeit soll abschließend ein Forschungsprojekt vorgestellt werden, dass mit seinem disziplinenübergreifenden Konzept einen Beitrag dazu leistet, 
inwiefern theoretische Ideale eine Bedeutung in der Praxis erfahren können und inwiefern es wertvoll ist, unterschiedliche Disziplinen miteinander zu verzahnen.

Das Forschungsprojekt, das für den Zeitraum von 2010 bis 2014 an der Universität Göttingen konzipiert wurde, zeigt mit seiner Vernetzung von Theorie und Praxis beziehungsweise Geisteswissenschaften und Medizin, einige Berührungspunkte zu dem hier vorliegenden Forschungsvorhaben. Unter dem Titel Autonomie und Vertrauen Schlüsselbegriffe der modernen Medizin wurden sieben Teilprojekte gegründet, die in unterschiedlichen Bereichen und auf unterschiedlichen Ebenen zu dem Begriff der Autonomie arbeiten. Vor dem Hintergrund der Hypothese, dass Selbstbestimmung in der modernen Medizin ein sehr hohes, im Alltag jedoch vom Scheitern bedrohtes Ideal ist, stand am Anfang des genannten Forschungsprojektes zunächst die Intention, Autonomie besser zu verstehen und das Verhältnis von Autonomie und Vertrauen zu beleuchten. Ein besonderer Fokus lag dabei auf Gruppen und Organisationen wie der Familie, den Patientenorganisationen und dem Krankenhaus. Erarbeitet wurden diese Fragestellungen in sieben Teilprojekten aus den Bereichen Philosophie (vgl. Steinfath 2016), Palliativmedizin (vgl. Nauck et al. 2016), Theologie (vgl. Anselm und Butz 2016), Recht (vgl. Duttge et al. 2016; vgl. Brauer und Lipp 2016) und Medizinethik (vgl. Wiesemann 2016; vgl. Jordan et al. 2016)4. Folgende Kernthesen sind Ergebnis des Teilprojektes Philosophie: Autonomie und Vertrauen stehen laut Studienergebnissen in einem Spannungsverhältnis zueinander, sind jedoch auch aufeinander angewiesen. Die medizinischen Praxis sollte demnach so gestaltet werden, dass Autonomie und Vertrauen sich wechselseitig verstärken können. Ein wesentliches Element von Vertrauen ist, dass den Vertrauenspersonen Ermessensspielräume gelassen werden. Bei dem Bestehen eines wechselseitigen Vertrauens gewähren sich Patienten und medizinisches Personal solche Spielräume gegenseitig (vgl. Steinfath 2016).

Als zweites sollen die für die vorliegende Arbeit relevanten empirischen Ergebnisse des Teilprojektes Medizin detailliert erwähnt werden: Zunächst wird aufgeführt, dass die während des gesamten Krankheitsverlaufs gemachten Erfahrungen eine zentrale Rolle für die Herstellung von Vertrauen in die Medizin und in die in ihr handelnden Personen spielen. Dabei ist ein einmaliges Vertrauen laut der Autoren leicht verletzbar und darf in Anbetracht dessen nicht als statisch angesehen werden oder gar als eine Form der Legitimation für einen rein paternalistischen Umgang benutzt werden (vgl. Nauck at al. 2016). Zudem führen die Autoren auf, dass Autonomie als Ergebnis einer 
zwischenmenschlichen Entwicklung zu verstehen sei (ebd.). Aus diesem Grund sollte Autonomie nicht als unabhängige Handlungsvorraussetzung von außen eingefordert oder erzwungen werden, da sonst das Recht auf Selbstbestimmung zu einer Selbstbestimmungspflicht würde (ebd.). Außerdem betonen die Autoren in ihrer Arbeit, dass Patientenautonomie immer an die Entscheidungen und an die Bereitschaft zur Umsetzung seitens der Therapeuten gebunden ist. Aufgrund dessen ist die Handlungsfreiheit des Patienten stets eingeschränkt und auf den jeweiligen medizinischen Handlungsrahmen begrenzt (ebd.).

Die Ergebnisse des vorgestellten Forschungsprojektes sind auf zwei unterschiedlichen Ebenen relevant für das vorliegende Forschungsanliegen. Zum einen liefert es hinsichtlich der Frage nach der Realisierbarkeit des Autonomieprinzips Material für verschiedene Reflexionsebenen. Und zum anderen bereichert es das Forschungsanliegen, indem es im Rahmen seiner Analysen stets den Bezug zwischen Theorie und Praxis herstellt.

\subsection{Der Einfluss des Konzepts auf hierarchische Strukturen}

Dort, wo sich Machtstrukturen innerhalb der Arzt-Patienten-Interaktion abzeichnen und Autonomiespielräume von Patienten begrenzen, spielen Kommunikation und die von Ärzten angewendeten Kommunikationsformen eine wesentliche Rolle. Vor dem Hintergrund der Reflexion, dass Kommunikation an sich ein sehr starkes Instrument in der Gestaltung der Arzt-Patienten-Beziehung und Grundlage für die Etablierung eines im Wandel begriffenen Arzt-Patienten-Verhältnisses ist, wird ihr im Rahmen der Ideale der Palliativmedizin ein höherer Stellenwert beigemessen als dies in anderen Fachbereichen der Fall ist. Parallel dazu besteht innerhalb des Fachbereichs der Palliativmedizin das Ideal, sensibler mit etablierten Machtstrukturen umzugehen und Strukturen der Kommunikation zu etablieren, in denen Hierarchien weniger Spielraum erhalten und die Patientenautonomie besser als in anderen Fachbereichen gewahrt werden kann (vgl. Becker und Xander 2008). In Hinsicht auf den klaren Fokus, Patientenselbstbestimmung zu fördern, die (medizinischen) Probleme der Patienten im Sinne eines interdisziplinären Konzepts anzugehen und einem multiprofessionellen Ansatz zu folgen, kann man die Ideale der Palliativmedizin auch als Ziel verstehen, Machtstrukturen innerhalb der ArztPatienten-Beziehung entschärfen zu wollen. 


\title{
II. Medizinethische Perspektive
}

\author{
4. Autonomie
}

\subsection{Autonomie bei Kant und Mill}

Der Terminus Autonomie entstammt dem Griechischen und setzt sich aus den Begriffen autos (selbst) und nomos (Gesetz) zusammen. Ursprünglich wurde unter diesem Begriff die innere und äußere politische Freiheit verstanden, was dem Recht der Stadtstaaten auf staatliche Unabhängigkeit und auf Setzung eigener Rechtsnormen gleichkommt.

Der Begriff der Autonomie in der philosophischen Tradition geht im Wesentlichen auf Immanuel Kant zurück, der Autonomie auf der Basis eines aufgeklärten, mündigen Selbstverständnisses des Menschen entwickelt hat (vgl. Kant 1968). In der Grundlegung der Metaphysik der Sitten entwickelte Kant seinen kategorischen Imperativ, der nach inm zufolge nicht einfach eine inhaltliche Norm ist, sondern das einzige Handlungs- und Normenprüfkriterium (ebd.).

Analog zu dieser Überlegung stellt auch das Autonomieprinzip innerhalb der Vorstellungen und Forderungen der Medizinethik ein Prüf- und Handlungskriterium dar. Trotz der mittlerweile vielfach vorhandenen kritischen Stimmen, die dem Autonomieprinzip entgegen gebracht werden, stellt dieses unverändert einen der zentralen Bezugspunkte des Argumentierens in der Medizinethik dar und wird auf verschiedenen Ebenen als eine Art Korrektiv angesehen.

Der kategorische Imperativ lässt sich im Wesentlichen auf folgende Formeln zurückführen: Die Naturgesetzformel, die Menschheitszweckformel und die Autonomieformel. Kant entwickelte die Naturgesetzformel, um vor dem Hintergrund der Eigenschaften und Einstellungen des Menschen zu bestimmen, wie dieser richtig zu handeln habe. Die Menschheitszweckformel besagt, dass der Mensch niemals nur Mittel zum Zweck sein darf, sondern stets auch als Zweck an sich selbst betrachtet und behandelt werden muss (ebd.).

Auch die Autonomieformel weist auf einen weiteren grundsätzlichen Aspekt der Kantischen Ethik hin. Der Mensch ist mit Hilfe seiner Vernunft in der Lage zu entscheiden, was er tut. Diese Tatsache setzt einen eigenbestimmten Willen voraus, der die Handlung des Menschen beeinflusst. Der Wille ist jedoch nur autonom, wenn er nicht fremdbestimmt (heteronom), sondern frei ist. Mit der Intention, allein aus der Vorraussetzung menschlicher Vernunft die Frage zu beantworten, wie ein potentielles moralische Gesetz 
aussehen müsse, entwickelt Kant die verschiedenen Formulierungen des kategorischen Imperativs. Zum einen nur nach der Maxime zu handeln, durch die man zugleich wollen kann, dass sie allgemeines Gesetz werde (vgl. Kant 1968), und zu dem nach Kant zufolge äquivalenten Gebot, stets so zu handeln, dass man die Menschheit jederzeit zugleich als Zweck und niemals bloß als Mittel braucht (ebd.).

Der Philosoph Hubert diskutiert den Aspekt, dass die Autonomieformel über die anderen Formeln insofern hinausgeht, als dass sie nicht nur die Allgemeinheit der Maxime, sondern zudem auch das Zustandekommen der Maximen aus Freiheit mit sich bringt.

Es ist hier nicht mehr einfach von einer Maxime oder einem Gesetz die Rede, sondern von dem das Gesetz selbstgebenden Subjekt. Der Autonomie-Formel zufolge geschieht die Gesetzgebung zum einen als allgemeinverbindlich (insoweit sind die Universalisierungsformel und die Naturgesetzformel aufgenommen) und zum anderen aus selbstbestimmter Freiheit. (Huber 2010, S. 12)

Zudem ist der Mensch nach Huber autonom, wenn er in seinem Denken und Handeln nicht nur seine eigene Freiheit und Selbstbestimmung achtet, sondern auch die der anderen. Freiheit ist Selbstbestimmung unter der Vorraussetzung, dass die Gleichrangigkeit der Selbstbestimmung aller geachtet wird:

[...] denn Unabhängigkeit von den bestimmenden Ursachen der Sinnenwelt (dergleichen die Vernunft jederzeit sich selbst beilegen muss) ist Freiheit. Mit der Idee der Freiheit ist nun der Begriff der Autonomie unzertrennlich verbunden, mit diesem aber das allgemeine Prinzip der Sittlichkeit, das besagt, dass ich meine Maxime im Gebrauche der Mittel zu jedem Zweck auf die Bedingung ihrer Allgemeingültigkeit als eines Gesetzes für jedes Subjekt einschränken soll. (Kant IV, 43 zit. nach Huber 2010, S.15)

Was der kategorische Imperativ gemäß der Autonomie-Formel also vorschreibt, ist die Freiheit aller Subjekte. Insofern schließt die Autonomie-Formel das ein, was in der Zweckan-sich-Formel ausgesprochen wurde: Gegenstand, Inhalt oder Zweck des unbedingten oder sittlichen Sollens ist keine beliebige Maxime, sondern die Maxime des vernünftigen Wesens $^{5}$ (vgl. Kant 1968).

5 Vernunft ist nach Huber die Fähigkeit, alle überhaupt möglichen Zwecke verstehend zu erfassen. Insoweit der Mensch Vernunftwesen ist, hat er Teil an dieser Fähigkeit und ist so in der Lage, die Zwecke (Sachen, Inhalte) in der Welt theoretisch-vernünftig zu erkennen und sie praktisch-vernünftig anerkennend als Güter zu würdigen (vgl. Huber 2010). 
Mill versteht unter der Autonomie eines Menschen etwas anderes als Kant. Im Fokus steht bei ihm die „individuelle Freiheit“ mit „anti-paternalistischer“ (Wils 1999, S.188) Haltung. In seiner Abhandlung On Liberty definiert Mill die bürgerliche oder soziale Freiheit bzw. die Frage nach dem Wesen und den Grenzen der Macht, welche die Gesellschaft rechtmäßig über die Individuen ausüben darf. In erster Linie geht es inm um die Begrenzung der Regierungsgewalt, sowie der Gewalt der Gesellschaft über den Einzelnen. Sowohl die politische als auch die soziale Freiheit des Individuums ist nach Mill gefährdet, da sie in wachsendem Maße von der Gesellschaft eingeschränkt wird. Damit stellt Mill das Verhältnis von Staat und Gesellschaft zum Einzelnen in den Mittelpunkt seiner Überlegungen. Um die Grenze der rechtmäßigen Machtausübung der Gesellschaft über das Individuum festzulegen, formuliert er einen Grundsatz, der als Freiheitsprinzip bezeichnet wird:

Dieses Prinzip lautet: dass der einzige Grund, aus dem die Menschheit, einzeln oder vereint, sich in die Handlungsfreiheit eines ihrer Mitglieder einzumengen befugt ist, der ist: sich selbst zu schützen. Dass der einzige Zweck, um dessentwillen man Zwang gegen den Willen eines Mitglieds einer zivilisierten Gemeinschaft rechtmäßig ausüben darf, der ist: die Schädigung anderer zu verhüten. Das eigene Wohl, sei es das physische oder das moralische, ist keine genügende Rechtfertigung. Man kann einen Menschen nicht rechtmäßig zwingen, etwas zu tun oder zu lassen, weil dies besser für ihn wäre. [...] Nur insofern sein Verhalten andere in Mitleidenschaft zieht, ist jemand der Gesellschaft verantwortlich. Soweit er dagegen selbst betroffen ist, bleibt seine Unabhängigkeit von Rechts wegen unbeschränkt. Über sich selbst, über seinen eigenen Körper und Geist ist der einzelne souveräner Herrscher. (Mill 1974, S.16)

Nur die jeweils betroffene Person selbst bestimmt nach Mill über das eigene Wohl, niemals ein anderer. Nur derjenige, der als souveräner Herrscher über sich selbst entscheidet, weiß, was er für sein Lebensglück benötigt und welches seine Präferenzen sind.

Jeder schützt seine eigene Gesundheit, sei sie körperlicher, geistiger oder seelischer Natur, am besten selbst. (ebd., S. 21)

Über die Art und Weise, wie Menschen diesen Ratschlag befolgen, können und müssen sie selbst entscheiden. Dies ist ein Aspekt, den nach Mill niemand anderes beurteilen 
kann. Autonome Entscheidungen führen, folgt man Mills Theorie, am ehesten zum eigenen Glück.

Um sich im Verlauf dem Verwirklichungshorizont von Autonomie annähern zu können, widmet sich die Arbeit im folgenden einer Auswahl der verschiedenen, für die Medizintechnik prägenden Autoren, die das Autonomieprinzip über die bisher vorgestellte ursprüngliche Perspektive hinaus beleuchten.

\subsection{Das Prinzip der Autonomie im Diskurs der angewandten Bioethik}

Das Prinzip der Autonomie, wie es aktuell als ein zentraler Bezugspunkt des Argumentierens in der (Medizin)ethik verwendet wird, lässt sich in seinen Grundzügen zwar auf den von Kant entwickelten Begriff zurückführen, wird jedoch im heutigen Diskurs in dieser Form nicht mehr verwendet. Die etablierte medizinethische Vorstellung von Autonomie ist die einer individuellen Entscheidungshoheit in Fragen persönlicher Belange und steht damit, wie Schöne-Seifert formuliert, eher in der liberalen Tradition der Theorie Mills als in der Tradition des kantischen Autonomieverständnisses (vgl. Schöne-Seifert 2007).

In seiner praktischen Anwendung in der modernen Bioethik gehen die Perspektiven auf das Prinzip der Autonomie jedoch über beide Autoren weit hinaus. Sowohl in den verschiedenen Strömungen der Philosophie als auch in der Medizinethik herrscht mittlerweile ein sehr fein ausdifferenzierter Diskurs über das Verständnis und die Auslegung des Autonomieprinzips. Einer der vielschichtigen Gründe für die Ausdifferenzierung in der Medizinethik ist, dass der informed consent (siehe Kapitel 6.3), der im konkreten Praxisgeschehen als beispielhaftes Strukturelelemt für den Fokus auf die Patientenautonomie steht, und das damit verbundene Ideal des aufgeklärten und eigenständig entscheidenden Patienten nicht unhinterfragt geblieben ist. Die konkrete Kritik an einem universal gültigen Autonomieprinzip ist vielfältig, da insbesondere in der Praxis des Entscheidens deutlich wird, dass eine vollständig unbeeinflusste und unabhängige Entscheidung von Patienten ebenso wenig der Regel entspricht wie das Ideal der vollständigen Aufklärung des Patienten (vgl. Woydack und Inthorn 2013). Ein weiterer Kritikpunkt ist die Betrachtung des Autonomieprinzips als ein zu individualistisches Konzept, dass mehr als Abgrenzungskonzept betrachtet wird und nicht als ein Konzept, das zusätzlich an eine Identität gebunden ist (vgl. Illhard 2008). Als eine zusätzliche Problemstellung der Autonomiedebatte wird unter anderem von Claudia Wiesemann diskutiert, dass das Autonomieprinzip in seinem jeweiligen Kontext zu betrachten und auszulegen sei. 
Es geht vielmehr um die Fragen der Einordnung dieses Prinzips in einen größeren moralischen, gesellschaftlichen und politischen Kontext, um ihm durchaus in kritischer Auseinandersetzung - eine Tiefendimension zu verleihen. (Wiesemann 2012, S. 3)

Die aktuellste Auseinandersetzung in der Literatur, die das Prinzip der Autonomie aus philosophischer, rechtlicher, theologischer und klinisch-praktischer Perspektive beleuchtet und somit einen kritischen Beitrag $\mathrm{zu}$ dem bestehen theoretischen Diskurs und der Relevanz für das Praxisgeschehen liefert, stammt von Wiesemann und Simon. Im Zentrum ihrer interdisziplinär angelegten Arbeit stehen folgende Aspekte:

Erstens muss der Begriff der Patientenautonomie mit Bezug auf die deutsche wie internationale Debatte theoretisch in seinen verschiedenen Facetten erfasst werden. Dabei leisten Philosophie, Recht und Theologie wesentliche Beiträge. Zweitens bedarf der Begriff kontextspezifischer Differenzierungen. Wie wird Patientenautonomie in der klinischen Praxis umgesetzt? Welche Probleme treten dabei auf? Drittens gilt es, das Verhältnis von Autonomie zu sozialen Bezügen auszuloten. (Wiesemann und Simon 2014, S. 9)

Kernpunkt des Diskurses, der über den kurz angerissenen Beispielen steht, ist, wie bereits erwähnt, die Kritik an einer universal gültigen Auslegung des Prinzips der Autonomie. Die damit einhergehenden Ansätze, die im Folgenden vorgestellt werden, fragen alle nach einer situations- und problembezogenen Betrachtung des Autonomiebegriffs, die über die traditionellen Perspektiven hinausgehen und sich als Konzepte unter dem Begriff der relationalen Autonomie zusammenfassen lassen.

Nicht ungeachtet dessen muss festgehalten werden, dass jedoch kaum ein anderes Konzept einen vergleichbar prägenden Einfluss auf die medizinische Praxis, ihre Handlungsakteure und die Entwicklung der Medizin- und Bioethik genommen hat, wie das Prinzip der Autonomie. Um einen chronologischen Überblick über die Entwicklung des bioethischen Diskurses zu dem Prinzip der Autonomie zu erhalten, folgt vor der konkreten Betrachtung der Konzepte zur relationalen Autonomie, zunächst ein Blick auf drei weitere für die Bioethik einschlägige Autoren, welche - obwohl sie sich auf einen eher kantisch ausgelegten Autonomiebegriff berufen - bis heute Bezugspunkte grundlegender Argumentationslinien und kohärentistischer Analysen bilden. In den folgenden zwei Kapiteln widmet sich die Arbeit in diesem Zuge dem Werk von Beauchamp und Childress und den Auslegungen des Autonomiebegriffs von Feinburg. 


\subsection{Die Prinzipienethik von Beauchamp und Childress}

Das 1979 erschienene und seither in verschiedenen Neuauflagen herausgegebene Werk Principles of Biomedical Ethics von Tom Beauchamp und James Childress hat sich als Standardwerk der Medizinethik etabliert. In ihrem Werk entwickelten die Autoren vier ethisch-moralische Prinzipien, welche Autonomie, Benefizenz, Nichtschaden und Gerechtigkeit sind (zitiert nach der 4. Auflage 1994). Beauchamp und Childress bezeichnen die vier Prinzipien als Prinzipien mittlerer Reichweite, die sich dadurch auszeichnen, dass es moralische Regeln sind, über deren Geltung erfahrungsgemäß ein großes Maß an Übereinstimmung hergestellt werden kann und die mit unterschiedlichen Begründungsansätzen vereinbar sind. Je nach Kontext oder Anwendungsgebiet bedürfen diese sogenannten mittleren Prinzipien jedoch ein gewisses Maß an Konkretisierung, sodass aus ihnen dann im Rekurs auf die Praxis sehr kontextspezifische Normen entwickelt werden. Die Prinzipienethik versucht also eine auf Kohärenz und Konsensfähigkeit angelegte pragmatische Normierung zu formulieren, bei der die Frage der Letztbegründung bewusst ausgeklammert wird.

Die vier Prinzipien unterliegen keiner hierarchischen Ordnung, sondern sind in ihrem Ausgangspunkt gleichwertig zu behandeln. Im ersten Schritt dienen sie zur Identifizierung, Analyse und Strukturierung ethischer Probleme und sollen an dieser Stelle in ihrer Bedeutung näher erläutert werden.

\section{Respekt vor der Patientenautonomie}

Wie in Kapitel 4.1 gezeigt wurde, existieren in der philosophischen Tradition verschiedene Auslegungen des Autonomiebegriffs. Nach Beauchamp und Childress lässt sich der Respekt vor der Patientenautonomie sowohl mit Kant als auch mit Mill begründen, obwohl es sich hierbei um zwei divergierende Theorien handelt (vgl. Beauchamp und Childress 1994, S.125). Generell hat der Begriff der Autonomie bei Beauchamp und Childress eine zentrale Rolle. In ihrem Ursprungswerk wurde der Begriff der Autonomie wie folgt definiert:

\section{Autonomy is a form of personal liberty of action where the individuell determines his or her own course of action in accordance with a plan chosen by himself or herself. (Beauchamp und Childress 1979, S. 56)}

Erst im Verlauf diskutieren die Autoren, dass das Prinzip der Autonomie nicht ausschließlich in dieser Eindeutigkeit zu fassen sei und innerhalb einer bestimmten Theorie zu konkretisieren ist (Beauchamp und Childress 1994, S. 120 f.) An Kants Theorie 
angelehnt bleibt der Aspekt des menschlichen Willens ein zentrales Element, der sich in einem sozialen Kontext manifestiert. Doch wie bereits erwähnt, wollen die Autoren Autonomie in einem weiter gefassten Sinne verstanden wissen. Die Autonomie des Anderen nicht zu respektieren, würde bedeuten, seine Freiheit zu missachten. Auch nach Beauchamp und Childress verweist die Autonomie auf die Vernunftsfähigkeit des Menschen. Wo diese nicht vorhanden ist, ist die Autonomie substantiell eingeschränkt.

In Hinblick auf den Kontext der medizinische Praxis müssen laut der beiden Autoren drei Bedingungen erfüllt sein, damit man definitiv von einer autonomen Patientenhandlung sprechen kann:

We analyze autonomous action in terms of normal choosers, who act intentionally, with understanding, and without controlling influences that determine their action. (Beauchamp and Childress 1994, S. 123)

$\mathrm{Zu}$ beachten ist allerdings, dass die genannten Bedingungen im konkreten Praxisgeschehen in unterschiedlicher Weise erfüllbar sind. Dass die Aspekte zum Teil sogar nur graduell erfüllbar sind, wird besonderes deutlich anhand der beiden Bedingungen, dass ein Patient die Problemlage vollständig verstehen und dass in keiner Weise Zwang ausgeübt werden soll. Beauchamp und Childress formulieren daher die Entscheidungsautonomie als einen graduellen Begriff:

Actions therefore can be autonomous by degrees, as a function of satisfying these two conditions to different degrees. (ebd.)

Aufgrund der Komplexität, unter der Entscheidungen für oder gegen eine medizinische Behandlung getroffen werden müssen, kann es generell nicht zielführend sein, stets die volle Autonomie einzufordern. Es es geht also vielmehr darum, vor dem Hintergrund der jeweiligen Dringlichkeit und Notwendigkeit der Situation, dem Patienten ein optimales Maß an Handlungsmöglichkeiten zu verschaffen.

Ein zentrales Strukturelement in der medizinischen Praxis, welches den Begriff der Autonomie fassbar macht, ist der informed consent. Im Zuge dessen soll die Selbstbestimmung des Patienten durch die Aufklärungspflicht des Arztes und das Zustimmungsrecht des Patienten im medizinischen Entscheidungskontext gesichert werden. Nach Beauchamp und Childress dient das Konzept des informed consent nicht nur dem Schutz des Patienten, sondern soll parallel dazu auch die individuelle Autonomie und eine rationale Entscheidungsfindung fördern, Zwang und Betrug verhindern und zur 
Selbstreflexion des medizinischen Personals beitragen (Beauchamp und Childress 1994, S. 142).

Auch wenn sich die vorliegende Arbeit in ihrem Vorgehen nicht explizit an der Prinzipienethik von Beauchamp und Childress orientiert, scheint es jedoch als ein Element der Analysegrundlage bereichernd zu sein, das Autonomieprinzip, wie es die beiden Autoren als einen dominant vorherrschenden Ansatz für die Medizinethik entwickelt haben, miteinzubeziehen.

\section{Nichtschädigung}

Das Prinzip des Nichtschadens, in welchem die Maxime des primus non nocere im Zentrum steht, verlangt, dass dem Patienten weder Leid noch Schaden zugefügt werden dürfen. Außerdem gilt es, dieses gleichzeitig auch zu verhüten und im Schadensfall Unheil aufzuheben. Die Absicht, keinen Schaden zufügen zu wollen ist dabei entscheidend. Ein wichtiger Aspekt in Bezug auf die Anwendbarkeit der vier Prinzipien ist dabei das Prinzip des Doppeleffekts. Erfahrungsgemäß ist es im Rahmen medizinischer Eingriffe teilweise nicht möglich Schaden in Form von Schmerzen zu verhindern. Eine Handlung ist im Sinne dieses Prinzips gerechtfertigt, wenn erstens die beabsichtigte Handlung sittlich legitimiert ist. Zweitens zählt die Intention der guten Wirkung, während die schlechte lediglich in Kauf genommen wird und nicht beabsichtigt ist. Drittens darf die schlechte Wirkung kein Mittel für die gute Wirkung sein und viertens muss die Verhältnismäßigkeit gewahrt bleiben (ebd. S. 207). Das beschriebene Prinzip des Doppeleffekts erlaubt also gegebenenfalls Schaden zuzufügen, um eine ursprünglich und über allen Nebenaspekten stehende gute Intention verfolgen zu können.

\section{Benefizienzprinzip}

Dieses Prinzip knüpft an das Prinzip des Nichtschadens an, geht gleichzeitig aber darüber hinaus. Es verlangt demnach nicht nur keinen Schaden anzurichten, sondern auch aktiv Wohltaten zu erzielen und zu gewährleisten. Gegenstand von Diskussionen ist, inwiefern Benefizienz eine Pflicht oder doch nur eine optionale Handlung des Guten sein kann. Trotz der Tatsache, dass hierzu unterschiedlichen ethische Positionen erhoben wurden, hat beispielsweise die medizinische Profession die Benefiezienz in ihrem Hypokritischen Eid zu einem Prinzip erhoben. Wichtiges Ziel ist dabei, eine Therapie zu finden, welche die Wohltat maximiert und dabei die negativen Konsequenzen und Effekte so gering wie möglich hält. Dabei ist es oftmals schwierig die verschiedenen Aspekte gegeneinander 
abzuwägen. Ein veranschaulichendes Beispiel, dass in Bezug auf diesen Punkt oftmals aufgeführt wird, sind Impfungen, da hier nicht nur die Einzelsituation, sondern auch die gesellschaftliche Gesamtsituation in Betracht gezogen werden muss. Eine weitere wichtige Fragestellung ist an dieser Stelle, wie man nach dem Prinzip der Benifizienz zu handeln hat, wenn sich der Wunsch des Patienten und das medizinisch Gebotene unterscheiden. Besonders relevant ist diese Frage für Patienten, dessen Urteilsfähigkeit durch Medikamenteneinfluss oder durch eine psychische Erkrankung beeinflusst ist. In derartigen Fällen kommt das Prinzip der Benefizienz mit dem Prinzip der Autonomie in Konflikt und man muss paternalistische Ansichten und deren Gegenpositionen je nach Kontext gegeneinander abwägen (vgl. Beauchamp und Childress 1994).

\section{Gerechtigkeit}

Das Prinzip der Gerechtigkeit diskutieren die Autoren mit Blick auf die Verteilung von medizinischen Gütern und Dienstleistungen. Gerechtigkeit, wie es in den Prinzipen von Beauchamp und Childress diskutiert wird, bedeute jedem das zu geben, worauf er Anspruch hat. Angelehnt daran steht also die Verteilungsgerechtigkeit im Vordergrund, welche die Frage mit sich bringt, inwiefern knappe medizinische Ressourcen gerecht an alle Betroffenen verteilt werden können. Ein zu diskutierender Punkt hinsichtlich dieser Frage ist, nach welchen Kriterien eine gerechte Behandlung tatsächlich erfolgen soll. Die Autoren bieten zu dieser Überlegung keine Lösung an, es bleibt also unklar wie sie die Integration von Gerechtigkeitsfragen in einen medizinethischen Diskurs sehen.

\subsection{Feinbergs mehrschichtige Herangehensweise an Autonomie}

Der amerikanische Sozialphilosoph Joel Feinberg unterscheidet 4 zentrale Bedeutungen von Autonomie (vgl. Feinburg 1986):

- Autonomie als persönliche Fähigkeit (capacity)

- Autonomie als situative Disposition (actual condition)

- Autonomie als Charakterideal (ideal of character)

- Autonomie als moralisches Recht (sovereign authority)

In den ersten beiden Bedeutungen ist der Begriff der Autonomie ein deskriptiver, in den letzten beiden ein normativer Begriff, der jedoch in seiner Normativität jeweils unterschiedlicher Beschreibungen bedarf. Autonomie als moralisches Recht ist etwas, was 
man einklagen kann. Autonomie im Sinne eines Charakterideals ist lediglich etwas, was eine Person anstreben kann oder etwas, das man ihr empfehlen kann. In der Sichtweise, in der Autonomie als moralisches Recht betrachtet wird, geht automatisch die Tatsache mit einher, dass der Mensch eine Pflicht zur Wahrung und Achtung der Autonomie anderer hat, während Autonomie als Charaktereigenschaft als solches zu nichts verpflichtet. Eine weitere Möglichkeit Feinbergs Autonomiebegriffe untereinander abzugrenzen findet sich in der Einteilung in Autonomie als eine Fähigkeit, wie es in den ersten drei Auslegungen der Fall ist, und in der Betrachtung von Autonomie als ein Anspruch, wie es die vierte Beschreibung Feinbergs mit sich bringt.

Anders als in der kantischen Sichtweise, in der der Mensch als vernunftsfähiges Wesen von Natur aus autonom ist, geht in der Betrachtung von Autonomie als Rechtsanspruch nicht automatisch die Selbstbestimmung jedes Individuums, beziehungsweise die Fähigkeit dazu, mit einher. Autonomie als Recht bedeutet lediglich auf einer theoretischen Ebene, dass der Mensch ein Recht auf Selbstbestimmung hat, dass also bestimmte Dinge nicht gegen oder ohne seine Zustimmung mit ihm geschehen dürfen.

Neben der Tatsache, dass mit der Etablierung des informed consent Autonomie als Recht in dem Praxisgeschehen des Medizinsystems vielleicht die wichtigste Rolle zugesprochen werden muss, spielen prinzipiell jedoch alle vier Auslegungen des Autonomiebegriffs eine Rolle für die Arzt-Patienten-Interaktion. Autonomie im Sinne einer Fähigkeit (als persönliche Fähigkeit (capacity) und als situative Disposition (actual condition) in Hinblick auf die Entscheidungsfähigkeit des Patienten, Autonomie als Ideal im Sinne einer reflektionsfähigen Arzt-Patienten-Beziehung und, wie bereits erwähnt, Autonomie als Rechtsanspruch des Patienten als normative Grundlage für oder gegen eine von ärztlicher Seite aus empfohlene medizinische Intervention. Ein wesentlicher Punkt bei der Betrachtung der vier Autonomiebegriffe Feinbergs ist, dass Patientenautonomie im zuletzt genannten Sinne nicht von den vorangegangenen Bedeutungen abhängig gemacht werden kann. Auch die Personen, die die Bedingungen der Autonomie als Fähigkeit oder als Ideal gar nicht oder nur eingeschränkt erfüllen können, haben den gleichen Rechtsanspruch auf Selbstbestimmung wie alle anderen, die sie erfüllen können.

Die insgesamt eher kantisch ausgelegte Sichtweise auf das Prinzip der Autonomie steht als ethisches Prinzip für sehr praktische, individuelle Patientenrechte im Kontext konkreter interindividueller Arzt-Patienten-Interaktionen. Trotz der unterschiedlichen Begründungen muss Feinbergs Konzept für den Begriff der Autonomie mehr als ein Abwehrrecht des Patienten gelesen werden und dient weniger als Korrektiv oder Handlungsvorschlag für das zunehmend komplexe Medizinsystem und seine Akteure. Hinsichtlich dieses Aspekts 
muss die Tatsache Erwähnung finden, dass Feinberg selbst darauf hingewiesen hat, dass die ursprüngliche Bedeutung von Autonomie vermutlich aus dem politischen Kontext von Staaten entstammt, und individuelle Autonomie demnach vielleicht nur eine politische Metapher sei (vgl. Feinburg 1986).

\subsection{Autonomie aus relational feministischer Perspektive}

In der Tradition Kants muss über die Art und Weise des menschlichen Handelns aus der Sicht einer unparteiischen und objektiven Perspektive, sowie aus der praktischen Vernunft heraus entschieden werden. Diese Vorstellung eines autarken, isolierten und frei von Bindungen existierenden autonomen Selbst wird heute als Ausdruck einer androzentrischen symbolischen Ordnung kritisiert, die jede Form von Abhängigkeit als Bedrohung von Autonomie versteht. Diese Konzeption ist häufig mit einer geschlechterhierarchischen dualistischen Denkweise verknüpft, demzufolge auch eine als weiblich definierte (und durch ihre Beschreibung abgewertete) Gegenseite existiert. Dieser sogennanten weiblichen Seite werden Attribute, wie Eingebundensein, Bezogenheit und Emotionen zugeordnet. Blickt man parallel dazu in die klassisch weiblich geprägten Berufsfelder und somit in die traditionellen Erfahrungsbereiche von Frauen, so findet man in dem Bereich der Versorgung und der Pflege klassische Felder, die aus den traditionellen Konzeptionen von Autonomie ausgegrenzt werden (vgl. Novy 2012).

Aus dieser Betrachtung heraus stellt die feministische Kritik die Abgegrenztheit des autonomen Individuums im traditionellen Bild in Frage und kritisiert zudem das Ignorieren eines gewissen körperlichen Aspekts, der je nachdem, ob jemand krank oder gesund ist, eine erhebliche Rolle für die menschliche Integrität und somit für seine Autonomie spielt. Der Mensch soll somit aus einer zusätzlichen Sicht in der Welt verortet gedacht werden, sodass Körper, Emotionen und Beziehungen eine Rolle spielen (ebd.).

We can learn much about the fragility of autonomy from those who once fit most closely the prevailing conception of the autonomous subject but no longer do. The disintegration of their sense of self-sufficiency exposes weaknesses in the construction of the "healthy body" as integral and unified and unmasks the kinds of psychological devices normal people unwittingly employ to shield themselves from awareness of the frailty of their own sense of self. (Donchin 2001, S.375)

Der Aspekt der nicht nur geistigen und intellektuellen, sondern zusätzlich auch körperlichen Integrität des Menschen, ist in der feministischen Perspektive auf das Prinzip der Autonomie neu. Wie in der traditionellen Auslegungen des Autonomieprinzips zu lesen 
ist, wird bis dahin selbst in den Diskursen, in denen es explizit um die Autonomie des Patienten geht, nicht im Detail diskutiert, was der biographische Bruch, den eine schwere Erkrankung auslösen kann, mit der Persönlichkeit, dem Körpergefühl und somit mit dem Wunsch nach Selbstbestimmung macht.

So wirft Anne Donchins Auseinandersetzung mit Autonomie Kritik an einer universal gültigen Perspektive auf den Autonomiebegriff auf, der am ehesten als Konstrukt für einen gesunden und starken Menschen dienen kann und dabei Gefahr läuft, körperliche und psychische Schwächen zu untergraben. Die Autorin zeichnet in ihren Arbeiten ein deutlich kontextspezifisches Bild des Autonomieprinzips und knüpft an die feministische Kritik an, die ihren Ursprung in der Betrachtung und Einbeziehung von weiblichen Sichtweisen und weiblichen Erfahrungszusammenhängen hat. In der Konsequenz geht es aber nicht um spezielle Autonomiefragen für Frauen, sondern vielmehr um ein Infragestellen von einseitigen und universal gültigen Konzepten, die sowohl für Frauen als auch für Männer in einem realen Leben nicht funktionieren. Die feministische Kritik stellt somit die Gültigkeit der traditionellen Autonomieauffassung für alle gleichermaßen in Frage.

Ebenso diskutiert Donchin im Konkreten relationale Ansätze des Autonomiprinzips, in denen zufolge enge interpersonelle Beziehungen unabdingbar sind für die Autonomie einer Person. Dabei wird nicht der Wert von Autonomie oder die Orientierung an dem Prinzip der Autonomie an sich infrage gestellt, sondern gleichzeitig anerkannt und bedacht, dass Menschen immer in Interaktion mit anderen stehen. Besonders mit Blick auf die Autonomie des Patienten in der Arzt-Patienten-Interaktion diskutiert sie drei Modelle der zwischenmenschlichen Beziehung, nämlich „friendship“, „mothering“ und „sistering“ (Donchin 2001, S. 378).

Though I am convinced that such models have only limited application to patient care, if applied cautiously and selectively each can illumine particular dimensions of provider-patient relationships so that providers can better deploy their relational capacities to enhance the autonomy of their patients. (ebd., $S$. 378)

Unter der Perspektive eines relational gedachten Autonomieprinzips steht die Autonomie der einzelnen Person nicht im Gegensatz oder in Abgrenzung zu ihren Beziehungen, sondern es wird beides zusammen gedacht und miteinander verflochten betrachtet. Kerngedanke ist dabei die Abkehr von einer absolut gedachten Autonomie, hin zu einer Orientierung an der Praxis. Autonomie soll nicht entweder als gegeben oder als nicht gegeben betrachtet werden, sondern ist unter einer relationalen Sichtweise stets in 
unterschiedlichem Ausmaß gegeben und dabei abhängig von der konkreten Situation und des jeweiligen Handlungskontextes.

Dieser Aspekt findet sich auch in der Care - Ethik wieder, in deren Kontext historisch gesehen weibliche Erfahrungen und Werte wie Liebe, Beziehung und Fürsorge eine höhere Bedeutung beigemessen wird. Der Mensch wird auch hier als grundlegend in Beziehungen eingebunden gesehen und Autonomie als relationale Autonomie konzipiert.

\subsection{Gestützte Selbstbestimmung}

Eine konkrete Auseinandersetzung mit dem relational verstandenen Autonomieprinzip liefert zudem Rainer Anselm. Er diskutiert eine Paradoxie, die sich im Zuge der hoch technologisierten Medizin im Umgang mit Patienten und dem Fokus auf Patientenautonomie ergibt und dessen Ansatz für die vorliegenden Forschungsfragen eine besonders fruchtbare Analysegrundlage darstellen: Es wird den Patienten innerhalb medizinischer Entscheidungsprozesse immer mehr Autonomie zugestanden, von der diese in Extremsituationen jedoch immer seltener Gebrauch machen können (vgl. Anselm 2002). Parallel zu der breiten Akzeptanz des Selbstbestimmungskonzepts ist auch die Sensibilität dafür gewachsen, dass das Prinzip deutliche Grenzen aufweist. Die moderne Ethik ist demnach gekennzeichnet durch die Kontroversen um die Überlegungen zu Freiheit und Selbstbestimmung, deren eindeutiger Vorherrschaft und durch ihre Ambivalenzen (ebd.). Anselm betont, dass nach dem stattgefunden „Begründungsdiskurs“ nun ein „Anwendungsdiskurs“ unabdingbar geworden ist (ebd., S. 73), da sich in der praktischen Anwendbarkeit des Prinzips stets „theorie-immanente Schwachstellen“ (ebd., S. 74) finden lassen. Das Selbstbestimmungskonzept führt nach Anselm, wenn es konsequent angewendet wird, zu einem für die moderne Gesellschaft typischen Wertepluralismus, der es schwer macht, noch verbindliche allgemeine Prinzipien zu formulieren (vgl. Anselm 2002). Gerade das Autonomieprinzip, wie es in der kantischen Auslegung zu lesen ist, greift jedoch nur in einem kleinen akademischen Gesellschaftsbereich und kann bei genauerer Betrachtung nicht auf moderne, durch vielschichtige Eigenschaften geprägte Gesellschaften übertragen werden. Hier gehen nach Anselm die Vorstellungen davon, was Selbstbestimmung bedeutet weit auseinander (ebd.). Der Autor diskutiert in diesem Zuge eine weitere Problematik, nämlich das „traditionelle Grundproblem ethischer Urteilsbildung“ (ebd., S. 75), welches die Schwierigkeit mit sich bringt, das eigene Verhalten und die persönlichen Entscheidungen mit dem Verhalten seiner Mitmenschen in Einklang zu bringen. Eine Fragestellung, die 
sich in einer Gesellschaft mit pluralistischen Wertvorstellungen sicher besonders stark stellt, da es in diesen weder einen gemeinsamen Bezugspunkt des Argumentierens noch gemeinsame verbindliche Normen gibt.

[...] dann müssen solche gemeinsamen Werte jeweils neu zwischen den Beteiligten ausgehandelt werden und die ethische Urteilsbildung kann nicht mehr - wie in einer kantischen Ethik - davon ausgehen, dass Selbstbestimmung als moralische Autonomie dazu führt, dass sich das einzelne Individuum auf dem Weg vernünftiger Argumentation den Normen unterordnet, die auch alle anderen als die allgemein zustimmungsfähigen Normen erachten. (ebd., S. 75)

Für das Zusammenleben in einer Gesellschaft muss also jeweils neu ausgehandelt werden, welche Formen von Selbstbestimmung generell existieren und wie die Selbstbestimmung des Einzelnen mit dem Interesse der Gesellschaft vereinbar ist. In dem heutigen Anwendungsdiskurs geht es also insgesamt vielmehr um eine „horizontale Dimension der Fragestellung“ (ebd., S. 76) als um eine vertikale Dimension, die die Autonomie- und Machtfragen zwischen „Obrigkeit und Untertan“ (ebd.) stellt. Hinsichtlich dieses Aspekts betont Anselm in Bezug auf die ethische Theoriebildung folgendes:

[...] der Großteil derer, die von den Orientierungsfragen des gesellschaftlichen Zusammenlebens betroffen sind, sind nicht fähig, am Diskurs über die Grenzen und Strukturen der eigenen Selbstbestimmung teilzunehmen, [und] dass es sowohl materielle als auch immaterielle Grenzen von Selbstbestimmung gibt. (ebd., S. 76)

In Hinsicht auf die immateriellen Grenzen von Selbstbestimmung diskutiert der Autor, dass das Paradigma der Selbstbestimmung in den medizinethischen Debatten immer häufiger versagt, weil genau in dem Moment, in dem ethische Konfliktfälle zu verhandeln sind, die Betroffenen ihr Recht auf Autonomie gar nicht oder nur unter Einschränkungen wahrnehmen können (vgl. Anselm 2002). Es wird also anhand derartiger Aspekte der eingangs erwähnte Konflikt im modernen Medizinsystem deutlich: Der wachsende medizinische Fortschritt führt zunehmend dazu, dass Ärzte entgegengesetzt zu der Intention des informed consent stellvertretend medizinische Fragen zu entscheiden haben, da ihre Patienten unter anderem aufgrund der Komplexität der medizinischen Sachverhalte nicht mehr zu einer Stellungnahme fähig sind. Den Überlegungen Anselms zufolge bedeutet dies stellvertretende Handeln jedoch gerade „keine Revision der 
Orientierung am Willen und an der Selbstbestimmung des Patienten“ (ebd., S. 77). Er argumentiert vielmehr in die gegenteilige Richtung und betont, dass der zugrunde liegende medizinische Fortschritt von dem Bemühen gespeist wird, den Patienten in zunehmend mehr Entscheidungs- und Extremsituationen die Rückkehr zu einem selbstbestimmten Leben zu ermöglichen (ebd.). Trotz der Tatsache, dass ein stellvertretendes (ärztliches) Handeln oder Entscheiden und die generell vorhandene Asymmetrie zwischen Betreuern und Betreuten die Gefahr mit sich bringen, dass gerade nicht das Autonomieprinzip, sondern eine paternalistische Tendenz erneut in den Vordergrund rückt, betont Anselm, dass Selbstbestimmung immer als „gestützte Selbstbestimmung“ zu entwerfen sei (ebd., S. 80). Um zu vermeiden, dass das Konzept der gestützten Selbstbestimmung zu einer „sublimierten Machtausübung“ entarte, schlägt der Autor vor, aus den Selbstbestimmungsentwürfen verschiedener Individuen möglichst einen „gemeinsamen Fluchtpunkt“ zu formulieren (ebd., S. 80). Zudem müssen Autonomiekonzepte immer als die Selbstbestimmung eines konkreten Individuums konkretisiert und explizit gemacht werden. Darin liegt laut des Autors der entscheidende Fortschritt in der Durchsetzung des Menschenrechtsgedankens in der Moderne (ebd.).

\subsection{Personale Autonomie}

Eine weitere Ausdifferenzierung, die sich im Rahmen des Diskurses zu dem Prinzip der Autonomie vollzogen hat, ist das Konzept der personalen Autonomie, welches in seinem Kern eine wesentliche Analysegrundlage für die Ergebnisse der vorliegenden Arbeit liefert. Entscheidungen werden als eingebettet in ihren jeweiligen konkreten Kontext verstanden, sodass sie von ihren jeweiligen Rahmenbedingungen her gedacht werden. Konkret bedeutet dies für Autonomie in der Arzt-Patienten-Beziehung, den Patienten vor dem Hintergrund seiner individuellen Situation zu sehen, die aufgrund der Krankheit durch spezifische Formen der Abhängigkeit und Einschränkungen gekennzeichnet ist (vgl. Rehbock 2002). Der Patient steht dabei in vielfältigen Bezügen und sozialen Beziehungen, die in der Entscheidung mit berücksichtigt werden. Neben dem Aspekt der Kontextualisierung spielen also zwischenmenschliche Beziehungen und die Persönlichkeit eines Individuums eine entscheidende Rolle. Beziehungen können die Entscheidungsfreiheit einer Person einschränken, ebenso können Entscheidungen aber unter Umständen auch erst ermöglicht werden, da „auch weitgehend einsichts- und entscheidungsfähige Patienten - für die Selbstsorge im Umgang mit ihrer Krankheit - auf Fürsorge angewiesen sind“ (Rehbock 2002, S. 145). Bestehende Beziehungen und über 
die Zeit gewachsene Vorlieben oder Einstellungen werden dann nicht als beschränkende Faktoren verstanden, sondern können auch als Unterstützung gedeutet werden, sodass Patienten auch in belastenden Situationen in der Lage sind, eine authentische Entscheidung auf der Grundlage ihrer Persönlichkeit und ihrer biographischen Identität zu treffen (vgl. Quante 2002). Quantes Idee zur personalen Autonomie und der Autonomie fördernden Dimension sozialer Beziehungen lässt sich jedoch nicht nur auf den Aspekt der Unterstützung zurückführen. Ein wesentlicher Aspekt, der das Konzept der personalen Autonomie mit geprägt hat, ist die Kritik an der implizit individualistischen Sichtweise des von Beauchamp und Childress geprägten Autonomiebegriffs. Eine Einflussnahme von außen wird in diesem Zusammenhang nur relevant, wenn die jeweilige Person in ihrer Entscheidung und Handlung nicht im Sinne von Zwang oder Manipulation beeinflusst wird. Es wird jedoch nicht explizit diskutiert, dass Personen immer in Beziehungen zu ihren Mitmenschen stehen, die durch eine Entscheidung genauso mit betroffen sein können. Auch bei ethisch betrachtet schwierigen Entscheidungsfragen oder dem biographischen Bruch durch eine schwere Erkrankung wurde bis hierhin mehrfach gezeigt, dass universal gültige Autonomiekonzepte in der Arzt-Patienten-Beziehung nicht (immer) funktionieren müssen. Quante schlägt diesbezüglich vor, dass bestimmte Fälle von medizinischem Paternalismus ethisch gerechtfertigt sein können, wenn sie der Wahrung der Persönlichkeit des Patienten dienen. Eine unabdingbare Vorraussetzung ist hierbei die Integration eines Konzepts von Persönlichkeit in das Konzept der Autonomie (ebd.). Auch Beauchamp und Childress diskutieren eine Form von personal autonomy, gehen in diesem Zusammenhang jedoch nicht in gleicher Weise auf die Rolle der (Selbst)Reflexion ein, wie sie in Quantes Arbeiten zu finden ist.

Persönlichkeit ist laut des Autors die individuelle Ausprägung der Personalität, die sich in der individuellen Biographie manifestiert. Wesentliche Elemente von Persönlichkeit sind beispielsweise Zeitbewusstsein, die Fähigkeit zur Kommunikation mit anderen und die Anerkennung anderer Individuen als Person (ebd.). Generell betrachtet Quante Persönlichkeit als Vorraussetzung für Autonomie. Ein Individuum setzt sich in ein Verhältnis zu prägenden sozialen Einflüssen und nimmt die Elemente und Faktoren in sein Selbstverständnis und somit als Teil seiner Persönlichkeit in die personale Autonomie mit auf (ebd.). In diesem Zusammenhang diskutiert der Autor das Verhältnis von personaler Autonomie und Authentizität. Er unterschiedet dabei zum einen eine „vorreflexive Authentizität“" und zum anderen die für das Konzept der personalen Autonomie bedeutsame „reflexive Authentizität“. Die so genannte reflexive Authentizität entspricht dem Ideal der personalen Autonomie und bedeutet für Quantes Konzept die kritische 
Reflexion aller Wünsche und Überzeugungen, sowie die Identifikation mit diesen (ebd., S. 293). Vorreflexiver Authentizität fehlt hingegen die kritische Reflexion - sie reicht nach Quante für Persönlichkeit aus, kann jedoch nicht zwangsläufig mit personaler Autonomie verbunden sein.

Quante liefert durch das Konzept der personalen Autonomie eine Möglichkeit, im Handlungs- und Entscheidungsgeschehen der kooperierenden Arzt-Patienten-Beziehung autonomiefördernde Maßnahmen durchzuführen, die auf den ersten Blick paternalistisch erscheinen. In diesem Sinne lässt auch dieses Konzept einen partiellen Verzicht auf Autonomie durch den Patienten selbst zu, was das vorliegende Konzept sehr praxisnah macht. Folgt man der Theorie des Autors, sind Patienten - im Optimalfall nach erfolgter Reflexion - in der Lage, Entscheidungen an anderen Person abzudelegieren ohne dabei ihre Autonomie zu verlieren. Grundlage dafür ist nach Quante stets die Personalität - also die Anerkennung der biographischen Identität (ebd.). 


\section{Der Begriff der Würde}

Die gesamtgesellschaftliche Debatte um das Thema Sterben und Tod ist stets von dem normativen Stellenwert des würdevollen Sterbens geprägt, welche die Forderung nach dem Erhalt der Würde und der Lebensqualität des Menschen am Lebensende mit sich bringt. Um ihre Intention zu verdeutlichen greift auch die Palliativmedizin den Aspekt des würdevollen Sterbens in ihren Idealen vielfach auf. Es bleibt jedoch auf beiden Ebenen offen, wie der Begriff der Würde in diesem Zuge theoretisch zu verorten ist.

Nähert man sich diesem Themenkomplex auf theoretischer Ebene, ist der würdevolle Tod eng mit dem autonomen Entscheiden bezüglich individueller Fragen am Lebensende verknüpft. Auch auf der von Ärzten genutzten sprachlichen Ebene kann der Tod nur in dem Fall würdevoll sein, wenn dem Patienten ein gewisses Maß an Selbstbestimmung eingeräumt wird.

Um diese normative Vokabel deutlicher einordnen zu können, beschäftigt sich das vorliegende Forschungsvorhaben im Folgenden mit ausgewählten theoretischen Ansätzen, aber auch mit den Schwierigkeiten, die mit den Vorstellungen des Würdebegriffs einhergehen.

\subsection{Die kantische Herangehensweise an die Würde des Menschen}

Kants Darstellung des Würdebegriffs hat den modernen philosophischen Diskurs der Menschenwürde maßgeblich geprägt.

Im Wesentlichen findet sich diese in Kants Moralphilosophie, wie er sie im zweiten Abschnitt der Grundlegung der Metaphysik der Sitten von 1785 entwickelt hat. Mit der Intention, allein aus der Vorraussetzung menschlicher Vernunft die Frage zu beantworten, wie ein potentielles moralische Gesetz aussehen müsse, entwickelt Kant die verschiedenen Formulierungen des kategorischen Imperativs. Zum einen nur nach der Maxime zu handeln, durch die man zugleich wollen kann, dass sie allgemeines Gesetz werde (vgl. Kant 1968), und zu dem nach Kant zufolge äquivalenten Gebot, stets so zu handeln, dass man die Menschheit jederzeit zugleich als Zweck und niemals bloß als Mittel braucht (ebd.).

Die Selbstzweckhaftigkeit der Personen unterscheidet diese deutlich von allem anderen, was es gibt und was bei Kant als Sachen bezeichnet wird. Auch Sachen kann man anstreben und bezwecken. Sie haben insofern also einen Wert für den Menschen, welcher jedoch immer auch in eine praktische Kalkulation einfließen kann, die dann unter Umständen zu dem Ergebnis gelangt, dass es besser wäre, die betreffende Sache zu 
Gunsten eines alternativen wertvollen Ziels aufzugeben. Diese Verrechenbarkeit des Werts aller Sachen bezeichnet Kant als ihren Preis (ebd.). Personen sind ebenfalls wertvoll, sogar notwendigerweise wertvoll, würde man aber ihren Wert gegen den einer anderen Person verrechnen, so würde man sie im Widerspruch zum kategorischen Imperativ lediglich als Mittel gebrauchen. Da man dies nach Kant niemals darf, sind sie nicht berechenbar. Ihr Wert ist demnach kein Preis, sondern das, was Kant in seinen Arbeiten als Würde bezeichnet.

Eine weitere kantisch geprägte Sichtweise auf den Begriff der Würde findet man bei Großmann. Er beschreibt die Tatsache, dass der Mensch von seinem Ursprung her bereits eine Würde innehat insofern, als dass er formuliert, es sei die „Würde [ist] durch die Vernunfttätigkeit und die Willensfreiheit gegeben.“ ist (Großmann 2004, S. 1089).

\subsection{Würde als erworbenes Merkmal}

Demgegenüber steht die Vorstellung, Würde existiere als erworbenes Merkmal menschlicher Existenz. Menschenwürde sei, so Luhmann, an die Bedingung „des Gelingens der Selbstdarstellung eines Menschen als individuelle Persönlichkeit“ geknüpft (Luhmann 1965, S. 61). Würde ist nach Luhmann demnach nicht an bestimmte ursprüngliche Merkmale des Menschen geknüpft, sondern erst im Laufe des Lebens über Lern- und Reifeprozesse erworben. Sie ist das Resultat eines Lebens in Verhältnissen, die es dem Menschen erlauben, menschenwürdig zu leben (Großmann 2004, S. 1089). Ergänzend führt Großmann grundlagenphilosophische Ansätze von Boethius auf, wonach Würde mit der Moralität des Menschen zusammen hängt - allerdings nicht mit seiner Moralfähigkeit, sondern mit der „Darstellbarkeit seiner moralischen Kompetenz“, seiner ethischen „protestas“. Würde ist demnach ein erworbenes, aufgrund „moralischer Habitualisierung auf die Natur des Menschen sich beziehendes Merkmal, das die innere oder charakterliche Qualität eines Menschen zum Scheinen bringt“" (Großmann 2004, S. 1090). „Die Würde ist nun - auf dem Wege eines moralischen Kommentars - als individuelle Leistung, also als ein erworbenes Merkmal statuiert.“ (ebd., S. 1090).

Betrachtet man zudem ausschnittsweise die Darstellung der Menschenwürde in der amerikanischen Literatur, wie sie beispielsweise in der Stanford Encyclopedia of Philosophy aufgeführt wird, zeigt sich, dass sich der Menschenwürdebegriff auf den Besitz von bestimmten kognitiven und emotionalen Kapazitäten gründet, die als moralisch wertvoll erachtet werden. Die den Würdebegriff prägenden Qualitäten variieren stark, beinhalten jedoch typischerweise die Möglichkeit zur autonomen Entscheidungsfindung, ein $\mathrm{Maß}$ an Urteilsvermögen und Argumentationsfähigkeit, die Partizipation an 
zwischenmenschlichen Beziehungen und ein $\mathrm{Ma} \beta$ an Empathiefähigkeit. Denjenigen Menschen, die über diese grundlegenden Würde begründenden Kapazitäten verfügen, kann demnach Menschenwürde zugesprochen werden (vgl. Karpowicz et al., The Stanford Encyclopedia of Philosophy (2009 Edition)).

Abschließend zeigt sich, dass sowohl der kantische als auch die verschiedenen dynamischen Ansätze zur Deutung des Würdebegriffs einen Bezug zur Autonomie des Menschen herstellen (siehe dazu auch Kapitel 4.1). Besonderes mit Blick auf Würde als ein erworbenes Merkmal scheinen die Grenzen des Würdebegriffs mit denen des Autonomiebegriffs sogar zu verschwimmen.

Die Menschenwürde ist nach Düwell et al. so zu bestimmen, dass sie die Möglichkeit zur autonomen Lebensführung für alle Menschen schützt. Die Bedingungen menschlicher Selbstbestimmung sind daher in dieser Konzeption dasjenige, was die Menschenwürde schützt. Geschützt werden demnach nicht bestimmte Ideale menschlicher Lebensführung, sondern die Ermöglichung ein selbstbestimmtes Leben überhaupt führen zu können (vgl. Düwell et al. 2002). Aus der Sichtweise und der Geschichte der Moralphilosophie wird deutlich, dass die Menschenwürde nicht ohne weiteres als subjektiv formulierter normativer Bezugspunkt gedacht werden kann. Vielmehr steht die Menschenwürde für eine menschenrechtlich orientierte Ordnung, die gegenüber anderen Ordnungen verteidigt werden muss. Dabei geht es auch darum, sowohl das Schutzgut als auch mögliche Strategien zu bestimmen, die die Menschenwürde gewährleisten können.

\subsection{Würdevolles Sterben im Rahmen der Rhetorik am Lebensende}

Besonders im ärztlichen Umgang mit Sterben und Tod fungieren sowohl der Würdebegriff als auch der Begriff der Lebensqualität eher als argumentatives Werkzeug für die Selbstbestimmung des Patienten am Lebensende. Diese Form der Rhetorik ist eng mit den aktuell viel diskutierten Assoziationen und Idealbildern zu dem sogenannten guten Sterben verbunden. Hinter Formulierungen, wie würdevolles Sterben und der Erhalt der Selbstbestimmung verbergen sich in der Realität komplexe Gefühle, die gerade von ärztlicher Seite aus schwer zu erwägen und auszudrücken sind. Die Kritik, sich eines gewissen Standardrepertoires an Rhetorik zu bedienen und Begriffe wie Würde im Patientengespräch nicht ausreichend zu klären und zu reflektieren, findet sich auch in der amerikanischen Literatur zum Umgang mit dem Thema Sterben und Tod. 
Importantly, I found that the rhetoric about dignity, suffering, and quality of life frames the way we consider the identity of persons who are near death. (Kaufmann 2006, S. 321)

Untersuchungen zeigen, dass der Begriff der Würde und der Begriff der Autonomie oder der Selbstbestimmung des Patienten bei Ärzten geläufig sind und vielfältig als Begründung für den sogenannte guten Tod oder das gute Sterben herangezogen werden. Es fehlt jedoch die Klärung, was sowohl von Seiten des Arztes als auch von Seiten des Patienten darunter verstanden wird und inwieweit die Begriffe auch handlungsrelevant werden können.

Neben dem Konzept Würde durch Selbstbestimmung wird in der Literatur diskutiert, dass Würde am Lebensende durch Fürsorge zu erhalten sei. Diese Vorstellung ist die grundlegende Idee hinter Chochinovs Konzept von der "dignity conserving care“, welche in einigen Zügen Überschneidungen mit der Palliativmedizin zeigt (vgl. Chocinov 2002a, S. 341; Chochinov et al. 2002b, S. 2258). Im Rahmen der Argumentation für die Stärkung der Autonomie des sterbenden Patienten ist das Konzept der Fürsorge jedoch auch mit einem kritischen Augenmerk zu betrachten, da dieses stark paternalistische Tendenzen hat. Bei einem derartigen Konzept steht die Selbstbestimmung des Patienten nicht nur im Hintergrund, sondern kann sogar im Widerspruch zu derselben stehen, da Fürsorgekonzepte stets unidirektional sind und keine Interaktion zwischen den Handlungsakteuren einschließen. Chochinov geht im Rahmen seiner Arbeit jedoch auch auf den viel diskutierten direkten Zusammenhang von Würde und Selbstbestimmung ein und formuliert in diesem Zusammenhang „Würde ist durch Selbstbestimmung zu erreichen und wird durch Fremdbestimmung gefährdet." (Chochinov et al. 2002a, S. 433) Im Rahmen der Studie Chochinovs werden Würde stärkende Therapien diskutiert, das heißt konkrete (medizinische) Interventionen, die die Würde des Patienten berücksichtigen und stützen sollen. Bei derart konkreten Bildern in Bezug auf einen sogenannten würdevollen Tod werden sehr subjektive Würdevorstellungen und -empfindungen formuliert, die nicht als allgemein gültig betrachtet werden können. Insbesondere in Hinsicht auf Bilder für den sogenannten guten Tod muss an dieser Stelle erwähnt werden, dass Bilder eines guten und würdevollen Todes, die den Anspruch der Allgemeingültigkeit erheben, Gefahr laufen, Sterben an sich zu normieren und die Handlungsspielräume in der Realität einzuschränken.

Eine weitere Gefahr, die der Entwurf eines zu positiven Bildes vom Sterben mit sich bringen kann, ist, dass das Sterben in der Öffentlichkeit in diesem Zuge in einer derart 
überhöhten Art und Weise betrachtet wird, dass kein Platz für ein weniger gutes Sterben bleibt und reale Bilder vom Sterben trotz des bestehenden Diskurses dadurch wieder tabuisiert werden.

Ein anderer wichtiger Aspekt, der hier Erwähnung finden muss, ist, dass die allgemein akzeptierte rhetorische Verknüpfung von Selbstbestimmung mit dem Erhalt der Menschenwürde am Lebensende im Praxisgeschehen häufig nicht funktioniert. Die Begründung dafür findet sich in der Tatsache, dass nicht jeder Mensch an seinem Lebensende bedingungslos autonom ist. Würdevolles Sterben kann also keinesfalls mit Vorstellungen von Autonomie gleichgesetzt werden.

Hinsichtlich eines subjektiven Empfindens von Würde in der Palliativsituation konnte eine Studie von Hack et al. zeigen, dass nur wenige Patienten, die am Ende ihres Lebens palliativmedizinische Betreuung erhielten, ihre Selbstbestimmung und ihre Würde angegriffen sahen (vgl. Hack et al. 2002, S. 700-708). Entsprechend dieser Definitionen und der wissenschaftlichen Ergebnisse wird die Palliativmedizin auch in den Medien als interdisziplinärer Behandlungsansatz dargestellt, der in der Lage ist, die Autonomie und die Würde des Menschen zu erhalten. Trotzdem sollte die Reflexion beider genannten Begrifflichkeiten nicht ausbleiben und weiterhin Gegenstand der Diskussion über die Realisierbarkeit von Idealbildern bleiben.

\subsection{Ansätze zur Problematik des Begriffs}

Auch wenn es in der Literatur viele Versuche gibt, den Begriff der (Menschen)würde eindeutig zu definieren und in einen klaren Kontext $\mathrm{zu}$ betten, scheint dies nicht vollkommen frei von kritischen Stimmen zu gelingen. Die amerikanische Medizinethikerin Ruth Macklin erhielt auf ihren Artikel Dignity is a useless concept beispielsweise eine sehr große Resonanz. Ihre Hypothese fasst die Autorin wie folgt zusammen:

[...] appeals to dignity are either vague restatements of other, more precise, notions or mere slogans that add nothing to an understanding of the topic. (Macklin 2003, S. 1419)

Die Autorin zählt damit zu denjenigen, die den Vorwurf formulieren, der Begriff der Würde werde lediglich aus rhetorischen Gründen und ohne einen wesentlichen argumentativen Gehalt verwendet. Macklin und ihre Befürworter sprechen sich in diesem Zuge dagegen aus, dem Begriff der Würde in Zukunft noch eine argumentative Sonderstellung in der Philosophie zukommen zu lassen. Während dieser Vorwurf zum Teil berechtigt und eine genaue Deutung des Begriffs schwierig erscheint, argumentiert Ralf Stoecker für den Sinn, 
der Menschenwürde weiterhin eine wichtige Rolle in der normativen Ethik einzuräumen. Er liefert in seinem Aufsatz Die Pflicht, dem Menschen seine Würde zu erhalten sowohl Erklärungen für die bestehende Skepsis als auch Argumente für den argumentativen Wert der Menschenwürde (vgl. Stoecker 2010). Seinen Ausführungen zufolge ist der erste Grund, kritisch gegenüber dem Rekurs auf die Menschenwürde zu sein, der verbreitete polemische Gebrauch des Begriffs, nämlich „der Verweis auf die Menschenwürde als Totschlagargument“ (Stoecker 2010, S. 100). Entgegnet man laut Stoecker seinem Gesprächspartner, er stehe mit seinen Ansichten oder Handlungen im Widerspruch zur Menschenwürde, „schließt man ihn zunächst automatisch aus dem Kreis der Anständigen aus“ (ebd.). In dem Fall, indem eine derartige Gesprächssituation unbegründet auftritt, ist sie unzweifelhaft kein argumentatives Werkzeug, sondern ein selbst intendierter Abbruch des Gesprächs.

Ein zweiter Aspekt, der gegen die Verwendung der Menschenwürde als Argument spricht, ist, von Macklin aufgeführt, nämlich die Verwendung des Begriffs aus religiöser Überzeugung (vgl. Macklin 2003). Nimmt man an, die Würde des Menschen begründe sich auf einer Art Gottesebenbildlichkeit, dann bestünde der argumentative Wert nur für die Menschen, die an Gott glauben (ebd.). An diesen Punkt knüpft Stoecker einen weiteren Aspekt an, der die Schwierigkeiten mit dem Begriff der Menschenwürde unterstreicht, nämlich die grundsätzliche Schwierigkeit, ihr einen systematischen Platz in der normativen Ethik einzuräumen. Er führt diesen Punkt auf die Annahme zurück, dass die Menschenwürde und die individuelle Autonomie des Menschen in Hinsicht auf ihre moralphilosophische Stellung in einer Art Konkurrenz zueinander stehen, bzw. dass die Stellung, die der Menschenwürde zukommen müsste, bereits mit dem, was man unter individueller Autonomie und den daraus resultierenden Menschenrechten versteht, besetzt ist (vgl. Stoecker 2010). Läge unser alleiniger Fokus und eine Art moralische Intention darin, ein „autonomes Wesen mit unveräußerlichen, universell gültigen Menschenrechten zu sein“ (ebd., S. 101) dann erscheint ein Berufen auf die Menschenwürde tatsächlich sinnlos. Als Gegenpol zu den genannten Schwierigkeiten, die ein Rekurs auf die Menschenwürde unsinnig wirken lassen, geht der Autor auf die wesentlichen Bereiche der medizinischen Ethik ein, in denen es selbstverständlich zu sein scheint, sich auf die Menschenwürde zu berufen. Stoecker führt hierzu Studien und Veröffentlichungen aus der Pflegeethik und der psychiatrischen Ethik auf, aus denen er exemplarische Aspekte hervorhebt, die für inn eindeutig eine Verletzung der Würde des Patienten darstellen. Zu Situationen, wie dem Missachten der Intimsphäre des Patienten, dem Missachten üblicher Konventionen des zwischenmenschlichen Umgangs (ältere Menschen oder psychisch 
Kranke werden beispielsweise wie Kinder behandelt) und dem Erschweren von Selbstbestimmung und Kontrolle formuliert der Autor:

Offenkundig ist die Bedrohung oder sogar Verletzung der Würde von Patienten ein ernstzunehmendes moralisches Problem im klinischen Alltag. Der philosophische Vorschlag, in der medizinischen Ethik ganz auf das „useless concept" Würde zu verzichten, mutet vor diesem Hintergrund seltsam weltfremd an. (vgl. Stoecker 2010, S. 102)

Der zweite medizinische Bereich, in dem immer wieder potentielle Würdeverletzungen thematisiert werden, ist die Psychiatrie. Beispielhaft führt Stoecker anhand von Studien das Reduzieren des Patienten auf seine psychiatrische Erkrankung, den Einsatz von Gewalt und Zwang gegenüber psychisch Kranken, das asymmetrische Verhältnis zwischen Therapeuten und Patienten, welches besonders in der Psychiatrie zum Missbrauch und zu Übergriffen verleiten kann und die Umgebung, in der psychiatrische Patienten zum Teil leben, als Würdeverletzungen auf (ebd.).

Menschenwürdeverletzungen existieren nach Stoecker jedoch nicht nur im Bereich medizinischer Kontexte und Situationen, sondern in allen Lebenszusammenhängen. Der Autor plädiert demnach klar dafür, dem Begriff der Menschenwürde in einer normativen Ethik einen klaren Wert und eine feste Rolle zukommen zu lassen. Der Autor wirft zudem die Frage auf, ob man einen neuen „ethisch gehaltvolleren Begriff“ (ebd., S. 106) braucht, oder ob es nicht reichen würde, sich auf die Rechte des Betroffenen zu berufen. Für die These argumentiert er damit, dass die klassischen Beispiele für Würdeverletzungen gleichzeitig auch Verletzungen moralischer Rechte sind. Bei der Betrachtung der geschilderten Beispielsituationen wird der Aspekt deutlich, dass in der Theorie jeder ein Recht darauf hat, gerade nicht in einer derartigen Art und Weise behandelt zu werden. Der Autor weist darauf hin, dass es bei der Beurteilung dieser Situationen in erster Linie also auf die Rechte einer Person ankommt. In Hinsicht auf die Rechte eines Individuums muss hier allerdings darauf hingewiesen werden, dass die Verletzung eines Rechts nicht auch gleichzeitig die Verletzung der Würde des Menschen darstellt (ebd.).

Geht man hingegen davon aus, dass es darüber hinaus noch ein Recht gibt, nicht entwürdigend behandelt zu werden, kommt man in der medizinischen Ethik nicht darum herum, sich darüber klar zu werden, worin der besondere Gegenstand dieses Rechts liegt, was es also heißt, die Würde des Menschen 
zu verletzen. Auch der Rückgriff auf die moralischen Rechte erweist deshalb die Würde nicht als philosophisch obsolet. (Stoecker 2010, S. 106)

Neben dieser eindeutigen Argumentation für den Wert und die klare Verankerung der Menschenwürde - sowohl in der Ethik als auch in der Philosophie - geht Stoecker zudem noch auf ein Modell ein, das ermöglicht, die Würde auf ihren unterschiedlichen Ebenen zu betrachten und einzuordnen. Man unterscheidet demnach zwischen der individuellen sozialen Würde, die beispielsweise im Krankenhaus in Gefahr gerät, und der fundamentalen Menschenwürde, wie sie in der allgemeinen Erklärung der Menschenrechte oder im Grundgesetz aufgeführt sind (vgl. Nordenfelt 2004).

Betrachtet man abschließend die aufgeführten Faktoren, welche die konkreten Vorstellungen von sozialer Würde bestimmen, weist Stecker explizit darauf hin, dass die potentielle Verletzung der Würde über Körper und Psyche hinausgehe und ebenso die Identität und damit verbunden die Würde des Menschen bedrohen könne (vgl. Stoecker 2010).

In einigen theoretischen Ansätzen wurde daraus die Konsequenz entwickelt, dass das Gebot, die Menschenwürde zu achten, auf ein Recht auf Würde hinaus laufen müsse (vgl. Schaber 2003). Stoecker selbst legt die Bedeutung des Gebots der Achtung der menschlichen Würde anhand drei konkreter Konsequenzen dar. Als erstes formuliert er das Gebot, die Menschenwürde zu achten, indem er sich auf die prima facie beruft, das der Mensch einen Anspruch darauf hat, nicht in seiner Würde geschädigt zu werden. In seinem zweiten Aspekt geht der Autor auf weitreichendere Dimensionen der Menschenwürdeverletzungen, wie Folter und Versklavung ein. Er wirft dabei einen Blick darauf, welche Chancen derartige Taten einem Menschen nachfolgend lassen, ihr Leben weiter zu führen, ohne in ihrer Integrität, ihrem Selbstbild und ihrer Würde tief verletzt zu sein. Er betont dabei, dass derartig weitreichende Entwürdigungen, die ein „anständiges Weiterleben" (vgl. Stoecker 2010, S. 112) unmöglich machen, auf keinen Fall gerechtfertigt sein können. An dritter Stelle formuliert er „positive Hilfsverpflichtungen“ (ebd., S. 112).

Da es zentral für unser Selbstverständnis ist, dass wir uns als Würdenträger behandeln, müssen wir auch bereit sein, uns wechselseitig dabei zu unterstützen, unsere Würde zu bewahren. Offenkundig ist dies bei Menschen, die noch nicht, nicht mehr oder überhaupt niemals in der Lage sind, sich um ihre eigene Würde zu kümmern. (ebd., S112) 
Das Verständnis von der sogenannten Pflicht, dem Menschen seine Würde als Grundbaustein und als Kern eines Menschenwürdeverständnisses zu erhalten, wie es auch in der Verfassung verankert ist, wird mit den Ausführungen Stoeckers somit deutlich plastischer.

\section{Die Arzt-Patienten-Beziehung}

\subsection{Die Entwicklung der ärztlichen Identität}

Der Kernpunkt des vorliegendes Forschungsvorhabens ist die Frage nach der Realisierbarkeit des Autonomieprinzips. Im Rahmen der Analysen hat sich gezeigt, dass dabei stets zwischenmenschliche Beziehungen und somit die in einer Beziehung agierenden Rollen(bilder) betrachtet werden.

Mit dem aktuellen Fokus auf das Modell des shared decision makings, dem in der Theorie die Förderung von Patientenselbstbestimmung zugrunde liegt, folgt die Medizin aktuell einer Art Idealbild für die Arzt-Patienten-Beziehung. Ein Leitbild der modernen ärztlichen Identität, das momentan gesellschaftlich akzeptiert zu sein scheint.

Die Frage, wie genau das moderne Arztbild konstituiert ist und wie daraus mögliche Problemfelder in der Arzt-Patienten-Beziehung zu bewerten sind, ist gerade für die medizinethische Reflexion von großer Bedeutung. Der Hauptgrund dafür zeigt sich darin, dass mit der Entscheidung für ein bestimmtes Idealbild zugleich eine moralische Entscheidung getroffen wird, die das Leben eines anderen betrifft (vgl. Maio 2008a).

Die vorliegende Arbeit konzentriert sich in ihren Auswertungen auf diesen Aspekt und fragt im weiteren Sinne auch nach dem Wert und der Realisierbarkeit von Idealen und Leitbildern - so wie das aktuelle Modell für die Arzt-Patienten-Beziehung formuliert wurde und wie auch der Fachbereich Palliativmedizin eine Schablone für ein gutes und selbstbestimmtes Lebensende entworfen hat. Im Rahmen einer ethischen Analyse (in der Medizin) ist man dabei in der Regel auf die Konkretisierung und die Reflexion bereits formulierter Idealbilder angewiesen (ebd.).

Bevor auf diesen Aspekt im Verlauf weiter eingegangen wird, widmet sich die Arbeit im Folgenden der Entwicklung der ärztlichen Identität, wie sie sich in der Vergangenheit vollzogen hat.

Eine wesentliche Rolle hat in den unterschiedlichen Epochen das Bild des Arztes als Berater und Erzieher gespielt. In der Antike und auch in der Renaissance wurde der Arzt als Erzieher in Fragen der Lebensführung und als Experte für die Fragen nach der 
Bewahrung der Gesundheit und sogar der Schönheit angesehen (vgl. Maio 2008a). Im späten 19. Jahrhundert wurde die Arztfigur primär als Berater für hygienische Angelegenheiten betrachtet. Generell kann man festhalten, dass die Rolle des Beraters, wie sie in der historischen Entwicklung gewachsen ist, heute von besonderer Aktualität ist. Insbesondere wenn es um die Themen Prävention und den Umgang mit chronischen Erkrankungen geht.

Eine andere Betrachtungsweise ist der Arzt als ein Freund seines Patienten. Eine Idealvorstellung, die im späten 18. und frühen 19. Jahrhundert entwickelt und in ihrem Selbstverständnis durch einen klaren Fokus auf Vertrauen geprägt wurde. Maio betont, dass in diesem Zuge eine bestimmte moralische Grundhaltung des Arztes in den Mittelpunkt gestellt wurde, bei der das persönliche Gewissen und die individuelle Moralität ausschlaggebend waren (ebd.). Dieses Bild des Arztes wandelte sich im Verlauf des 19. Jahrhundert stark. Der Grund war die Etablierung von Krankenhäusern als neuer Ort für die Arzt-Patienten-Interaktion. Das freundschaftliche Bild des Arztes wurde durch die wachsenden hierarchischen Strukturen innerhalb des Krankenhauses und durch eine autoritäre und distanzierte Arztfigur abgelöst. Verstärkt wurde diese Dynamik durch die zunehmende naturwissenschaftliche Orientierung der Medizin, im Zuge derer technisches Wissen von größerer Bedeutung war als eine Art persönliche Tugend im Rahmen des beruflichen Selbstverständnisses. Alle Formen der Krankheit wurden in dieser Entwicklung klarer objektivierbar und messbar, was dazu führte, dass die persönliche Meinung der Arztfigur als Freund in den Hintergrund rückte. Somit wurde der Patient im Zuge der naturwissenschaftlichen Neuorientierung zu einer Art Träger objektiver Symbole reduziert (vgl. Toellner 1991). Der Arzt als Freund wurde von einem Arzt als Techniker abgelöst, der hoch spezialisiert, perfektioniert und als einseitiger Experte arbeitete (vgl. Maio 2008a). Als Resonanz auf diese Dynamik im damaligen Medizinsystem entstanden jedoch auch Kontrastbilder zu dem Arzt als Techniker. Karl Jaspers diskutierte beispielsweise das Bild des Arztes als Schicksalsgefährten und nahm damit Abstand von einem Arztbild, das eine rein technisch und professionell arbeitende Figur zeichnete (vgl. Jaspers 1953).

Seit einigen Jahrzehnten wird zunehmend das Idealbild populär, welches den Arzt als eine Art Partner betrachtet. Die Gegenseitigkeit von Arzt und Patient soll in diesem Zusammenhang eine Vorraussetzung für das Gelingen einer partnerschaftlichen Beziehung sein, in der die Selbstbestimmung des Patienten immer stärker in den Vordergrund rückt. Wie die Ergebnisse des vorliegenden Forschungsvorhabens im Verlauf zeigen werden, ist jedoch zu diskutieren, ob tatsächlich jede medizinische Entscheidungssituation unter dem Paradigma der Partnerschaft funktionieren kann. Der 
mit einer derartigen Arzt-Patienten-Beziehung einhergehenden Fokussierung auf die Autonomie des Patienten wird in den aktuellen Diskursen ein unumstrittener Wert zugesprochen, es ist jedoch parallel dazu zunehmend Thema, dass es mit einer Überbetonung dieser sogenannten Partnerschaft auch zu einer Überforderung der Akteure des Praxisgeschehens kommen kann (vgl. Woydack und Inthorn 2013). Besonders deutlich wird dieser Aspekt bei Entscheidungen, die das Lebensende des Menschen betreffen, worauf im Verlauf der vorliegenden Arbeit noch im Detail eingegangen wird.

\subsection{Das medizinische Aufklärungsgespräch}

Das Aufklärungsgespräch für eine Erkrankung mit infauster Prognose sowie Gespräche mit sterbenskranken Menschen zählen zu den schwierigsten kommunikativen Aufgaben im Kontext der Arzt-Patenten-Interaktion. Studienergebnisse zeigen, dass eine gute Kommunikationsfähigkeit von Seiten des Arztes für schwer kranke Patienten noch vor dem Wunsch nach emotionaler Unterstützung die höchste Priorität hat (vgl. Wenrich et al. 2001).

Dabei zählen - neben einer grundlegenden Reflexionsfähigkeit über die eigene Art der Kommunikation - Stilmittel, wie das aktive Zuhören und dem damit verknüpften einfühlenden Verständnis und Techniken, wie die des Spiegelns eine wesentliche Rolle.

Als einer der grundlegenden Leitfäden für Kommunikation in der palliativmedizinischen Praxis gilt das SPIKES-Protokoll (vgl. Baile et al. 2000). Mit seiner klaren Struktur und seinen konkreten Vorschlägen, insbesondere für den Gesprächsrahmen, die Gesprächsinhalte und die Wahrnehmung der Patientenreaktionen kann der Leitfaden als Hilfestellung für die Gestaltung eines schwierigen Gesprächs dienen. Laut Franck und Sitte wird das prozesshafte Geschehen der Übermittlung einer infausten Prognose hierbei besonderes betont. Von wesentlicher Bedeutung ist zudem, dass dessen Geschwindigkeit und Maß letztlich der Patient steuern soll (vgl. Franck und Sitte 2013).

Hinter dem Akronym SPIKES stehen die Gesprächselemente Situation, Patientenwissen, Informationsbedarf, Kenntnisvermittlung, Emotionen ansprechen sowie Strategie und Zusammenfassung.

Neben der Vermittlung reiner Sachinformationen wie der medizinischen Diagnose, der Prognose und den jeweiligen Behandlungsoptionen liegt das Augenmerk also darauf, wie viele dieser Sachinformationen der Patient zum Zeitpunkt der Diagnosestellung versteht und verstehen sollte. Außerdem soll die besondere Lebenssituation des Patienten und seiner Angehörigen eruiert und mit ihren möglichen psychosozialen Ressourcen, aber 
auch Problemen in das Gespräch einbezogen werden. Mit Blick auf die allgemeine Gesprächsgestaltung soll dem Gespräch ein definierter Rahmen gegeben werden, ausreichend Zeit, die jedoch auch klar begrenzt werden darf, und ein Raum, in dem ungestört kommuniziert werden kann (vgl. Baile et al. 2000).

Im Folgenden soll das SPIKES-Protokoll genauer erläutert werden.

\section{Situation}

Um einem guten Gespräch einen angemessenen Rahmen zu geben bedarf es eines separaten Raumes und nicht beispielsweise das Zweibettzimmer im Krankenhaus. Außerdem schlagen die Autoren vor, den Patienten zu ermutigen, sich eine Vertrauensperson zu den Gesprächen mit zu bringen, die von Anfang an unterstützend dabei ist. Somit besteht die Möglichkeit, dass diese Vertrauensperson während des Gespräches unterstützend mitwirken kann und nach dem Gespräch als Dolmetscher des Gesprächsinhaltes und als Gedankenstütze wirken kann.

Der Arzt sollte sich während des Gesprächs auf die Sprache des Patienten einstellen und ganz auf inn konzentrieren können. Anhand dieses Aspekts wird die Notwendigkeit betont, sich dabei eigener Schwierigkeiten sowie Ängsten bewusst zu werden, um mit unvorhergesehenen Reaktionen des Patienten fertig werden zu können (vgl. Baile et al. 2000).

\section{Patientenwissen}

Bei dem Vermitteln von Informationen bezüglich der Diagnose und potentieller Probleme soll durch möglichst offene Fragen das oft bereits vorhandene Patientenwissen genutzt werden, um verständlich aufzuklären. Als Beispiel nennen die Autoren Fragen wie, „Was denken Sie, wie es jetzt um Sie steht?", „Was hat man Ihnen denn schon alles zu Ihrer Erkrankung erklärt?" Antworten auf derartige Fragen können einen guten Einstieg darstellen, auf denen das weitere Gespräch behutsam aufgebaut werden kann.

\section{Informationsbedarf}

Jeder Patient muss die Chance haben, soviel über seine Diagnose und auch seine Prognose zu erfahren, wie er möchte. Aber nicht jeder Patient will und muss dasselbe über Krankheit, Therapieoptionen und Prognose wissen. Elementar wichtig für das Gespräch ist dabei, dass der Aufklärende in der Lage ist, zu bemerken, wie viel Information der Patient in der jeweiligen Situation haben möchte und ertragen kann. Laut Franck und Sitte klären 
Ärzte anfangs tendenziell mit zu viel Informationen auf einmal auf, was leicht dazu führt, dass der Patient beim Beenden des Gesprächs nicht mehr alles versteht und sich später kaum noch richtig daran erinnern kann (vgl. Franck und Sitte 2013). Als Hilfestellung schlagen die Autoren folgende Fragestellungen vor:

„Wollen Sie es gleich wissen, wenn die Situation schlechter wird?", „Sind sie jemand, der gerne alles selber weiß oder möchten Sie lieber, dass ihre Kinder die Dinge regeln und Bescheid wissen?" Dabei weisen die Autoren darauf hin, dass kaum ein Patient die Information ausschließlich in den Händen seiner Angehörigen wissen möchte.

Ein wesentliches Augenmerk sollte auf die Sprache der aufklärenden Person gerichtet sein. Fachausdrücke, auch wenn sie präziser sind, sollten vermieden werden, wenn sie nicht sprachliches Allgemeingut sind. So machen Franck und Sitte den Vorschlag stets Vokabeln, wie „Adenokarzinom des Kolons“ durch „Darmkrebs“ zu ersetzen oder anstelle einer „malignen Erkrankung“ den Begriff „Krebs“ zu benutzen um die Diagnose für den Laien realistisch zu transportieren. Laut der beiden Autoren besteht die ärztliche Kunst darin, realistisch und authentisch über die Diagnose aufzuklären, den Patienten dabei aber nicht gleichzeitig jede Hoffnung zu nehmen. Als ein klassisches Beispiel wird die Aussage „Wir können nichts mehr für Sie tun!" aufgeführt, die in jedem Fall durch Formulierungen, wie „Wir können Sie wohl nicht heilen, aber wir können Ihnen sehr viel helfen, besser weiter zu leben, solange es geht." (vgl. Franck und Sitte 2013). Außerdem sollte frühzeitig angesprochen werden, welche Formen der Unterstützung die Lebensqualität verbessern können oder Ängste lindern können. In Hinsicht auf konkrete Ängste im Rahmen der jeweiligen Erkrankung (z.B. respiratorische Insuffizienz bei Amyotropher Lateralsklerose oder lleus beim inoperablen Rektumkarzinom) sollten rechtzeitig Lösungsansätze und potentielle Therapieoptionen besprochen werden.

\section{Emotionen}

Die von Patienten gezeigten Emotionen sollten reflektiert und angesprochen werden. Genauso dürfen Ärzte selber Gefühle zeigen, wenn zugleich auf die Signale und Reaktionen des Patienten und seines Begleiters geachtet und eingegangen wird. Mit Blick auf den Umgang mit Emotionen sollten die Aufklärenden die Gefühle des Patienten nicht relativieren, sondern innen so, wie sie zum Ausdruck gebracht werden, mit Verständnis begegnen. Verzweiflung, Angst oder Wut beispielsweise kann mit Hilfe von Rückfragen begegnet werden, um so an eine genauere Schilderung der Gefühlslage des Patienten zu gelangen (Franck und Sitte 2013). 


\section{Strategie und Zusammenfassung}

Häufig kann es so sein, dass beim ersten Gespräch nur wenige Sachinformationen vermittelt werden können und dass selbst wesentliche Kernaussagen sowohl vom Patienten und manchmal auch von dessen Begleitern falsch verstanden oder vollkommen umgedeutet werden. Offene Fragen am Gesprächsende oder die Aufforderung an den Patienten, zu schildern, was verstanden wurde können hier hilfreich sein. Sollte ein Patient bereits nach einem ersten Gespräch in der Lage sein, über das weitere Vorgehen nachzudenken, sollte dies mit dem Angebot der weiteren Begleitung und im Sinne von realistisch erreichbaren (Etappen-) Zielen formuliert und am Ende zusammengefasst werden (vgl. Franck und Sitte 2013). Außerdem ist es für beide Seiten sinnvoll, zeitnah einen weiteren Termin zu vereinbaren, an dem das Gespräch fortgeführt und vertieft werden kann.

Das SPIKES-Protokoll ist kein Konzept, das exakt immer in dieser Form angewendet werden muss, aber es gibt gerade dann viel Sicherheit für das Gespräch, wenn Ärzte über wenig Erfahrung verfügen. Außerdem kann es hilfreich sein, wenn das Gespräch für den Arzt vermutlich sehr belastend sein wird. Mit wachsender Erfahrung reichen lediglich Elemente des Protokolls aus und die aufklärende Person passt sich mit ihrer Gesprächsführung der jeweiligen Situation und der Persönlichkeitsstruktur des Patienten jeweils an.

In Hinsicht auf die ärztliche Schweigepflicht muss der juristische Aspekt beachtet werden, dass Dritte nur aufgeklärt werden dürfen, wenn der Patient dies zuvor explizit erlaubt hat. Sinnvoll ist es laut der Autoren immer, wenn nicht nur der Patient aufgeklärt wird. Das Szenario, in dem aus Rücksichtnahme oder aus kulturellen Gründen nur die Angehörigen vollständig aufgeklärt werden und der Patient nicht oder kaum, ist ein äußerst bedenkliches, aber häufig anzutreffendes Vorgehen. Auch aus palliativmedizinischer Sicht ist dies in fast allen Fällen abzulehnen.

Problematisch ist jedoch die Tatsache, dass laut Studienergebnissen jeder dritte Arzt sich grundsätzlich scheut, den Patienten über einen bösartigen Befund aufzuklären (Meredith 1996). Dies führt zu einer gehemmten Kommunikation nicht nur in der Arzt-PatientenBeziehung, sondern auch innerhalb der Familie des Menschen. Zudem wird der Patient damit komplett seiner Selbstbestimmung beraubt und es ist darüber hinaus rechtlich nicht 
legitim. Neben dem verfassungsrechtlich garantierten Recht auf Information besteht das Praxisproblem, dass ein Patient nicht in der Lage ist, rechtswirksam in eine notwendige Schmerztherapie, eine Tumorbehandlung oder einen Eingriff einzuwilligen, wenn er nicht aufgeklärt ist. Prinzipiell hat der Patient das Recht, so umfassend aufgeklärt zu werden, wie er es für richtig hält und wünscht. Für Ärzte besteht im Rahmen des Gesprächs trotz absoluter Offenheit zudem stets die Möglichkeit auch ein Stück Hoffnung in Teilaspekten zu vermitteln. Ein Element dessen wäre beispielsweise das Angebot, dass ein Palliativteam stets bereit ist, sich um Probleme zu kümmern und in aussichtslos erscheinenden Situationen Hilfen zu bieten.

\subsection{Der informed consent als kommunikatives Gleichgewicht}

Eines der zentralen normativen Kriterien für die moderne Arzt-Patienten-Beziehung ist die Einhaltung des informed consent (Partizipative Entscheidungsfindung). Dies beschreibt die Interaktion, beziehungsweise die Kommunikation zwischen Arzt und Patient, die zum Ziel hat, zu einer von Patient und Arzt gemeinsam verantworteten Übereinkunft über eine angemessene medizinische Behandlung zu kommen (vgl. Butzlaff et al. 2003). Trotz des bestehenden Machtgefälles zwischen den Akteuren der medizinischen Praxis soll die Autonomie des Patienten Kernelement dieser Umgangsform sein. Gewährleistet wird dies durch eine Art partnerschaftliche Beziehung zwischen Patient und Arzt mit einem möglichst gleichem Informationsstand über die medizinischen Behandlungsoptionen. Zu betonen ist jedoch, dass initial der Arzt derjenige ist, der die Behandlungsindikation stellt und daraufhin seinen Patienten entsprechend aufklärt, sodass eine Partizipative Entscheidungsfindung möglich wird. Dazu gehört das Aufzeigen verschiedener gleichwertiger, im besten Falle evidenzbasierter Behandlungsoptionen, sowie eine Erläuterung hinsichtlich der jeweiligen Vor- und Nachteile. Rechtlich gesehen gewährleistet dieses Modell der Arzt-Patienten-Interaktion die Möglichkeit für den Patienten, einem medizinischen Eingriff zuzustimmen oder inn bewusst abzulehnen.

Auch in Hinsicht auf die Selbstreflexion des Arztes ist es notwendig, dass sich dieser in der Lage sieht, sich von dem eigenen Interessenstandpunkt zu distanzieren, um in der Lage zu sein an einer gemeinsamen Praxis mitzuwirken (vgl. Nida-Rümelin 2009). Neben dem Versuch mit dem Modell des informed consent ein kommunikatives Gleichgewicht zwischen Arzt und Patient zu erzielen, gilt es jedoch auch für beide Seiten ein Gleichgewicht zwischen Eigenverantwortung und Autonomie zu ermöglichen. 


\subsection{Patientenverfügungen als kommunikatives Werkzeug}

Wie im vorangegangenen Kapitel bereits beschrieben, fungiert die Orientierung am Willen des Patienten aus Respekt vor dessen Autonomie als maßgebliche Norm bei medizinischen Entscheidungen. Auch in dem Fall, in dem es nicht um die Entscheidung für oder gegen eine medizinische Intervention geht, sondern um einen medizinisch-ethischen Konflikt, beispielsweise bei brisanten Fragen am Lebensende, zählt die Orientierung am Willen des Patienten. Besonders bei Fragen am Lebensende sind jedoch verschiedene Szenerien denkbar, in denen ein Patient aufgrund seiner Erkrankung oder aufgrund seiner aktuellen Lebensumstände das inm zugesicherte Recht auf Autonomie gerade zu diesem Zeitpunkt nicht ausüben kann, wenn es notwendig wäre. Hinsichtlich derartiger Situationen sollen Patientenverfügungen ermöglichen auch dort noch den individuellen Willen des Patienten als Maßstab gelten zu lassen, wo dieser ihn nicht mehr artikulieren kann. Seit dem Jahr 2009 ist die Patientenverfügung und insbesondere die Verbindlichkeit einer erstellten Verfügung nach deutschem Recht gesetzlich geregelt. Laut Bundesgesetzbuch ist eine existierende Patientenverfügung für Betreuer, Ärzte oder Bevollmächtigte unmittelbar verbindlich und gilt unabhängig von der Art oder dem Stadium der Erkrankung eines Patienten. Dem in der Patientenverfügung geäußerten Willen muss stets Geltung verschafft werden, wenn die Festlegung in der Patientenverfügung auf die aktuelle Lebens- und Behandlungssituation zutreffen (vgl. BGB 2008). Aktuell ist es jedoch nicht generell verpflichtend, eine Patientenverfügung zu verfassen, was in der Literatur kontrovers betrachtet wird. Beckmann diskutiert beispielsweise, dass die Patientenverfügung vor dem Hintergrund eines im Wandel begriffenen Arzt-PatientenVerhältnisses ein Erfordernis ersten Ranges sein müsse (vgl. Beckmann 1998). Er stützt sich bei diesem Vorschlag auf den Wunsch des Patienten, verstanden zu werden und eine kommunikative Verbindung zu seinem Arzt auch dann gesichert zu wissen, wenn er sich nicht mehr äußern kann (ebd.). In jedem Fall in dem ein behandelnder Arzt, die Familie oder ein Betreuer des Patienten lediglich den mutmaßlichen Willen des Patienten für eine Entscheidung heranzieht bedeute dies nach Beckmann Fremdbestimmung und somit das Gegenteil von Autonomie (ebd.). Hinsichtlich dieses Aspekts muss also klar zwischen dem Verfassen einer Patientenverfügung als primär autonomiefördernder Akt und der Situation, in der sich ein Patient tatsächlich nicht mehr äußern kann - und die formulierte Verfügung geltend gemacht werden soll - unterschieden werden.

Ein weiterer Aspekt, der an den genannten anknüpft, ist die Reichweite von Patientenverfügungen. Der Diskurs beinhaltet die Überlegung, ob eine einmal in einer 
Patientenverfügung dargelegte Entscheidung lediglich für den aktuellen Willen des Patienten spricht und eine therapieeinschränkende Wirkung nur in unmittelbarer Todesnähe greifen solle, oder ob eine solche Beschränkung von unbestimmter Reichweite gelten soll. In Hinsicht auf den biographischen Bruch, den eine schwere Krankheit mit sich bringt, könnte man schließlich in Erwägung ziehen, dass sich die Meinung eines Menschen in Bezug auf medizinische Interventionen noch einmal ändert. Neben den Diskussionen aus rechtlicher und aus ärztlicher Perspektive sind aus einer Studie von Rainer Anselm im Jahr 2008 interessante Ergebnisse hervorgegangen, die die Sicht der Patienten auf Patientenverfügungen beleuchten (vgl. Anselm 2008). In den Ergebnisse der Studie wird deutlich betont, dass Patientenverfügungen darauf abzielen, die strukturelle Asymmetrie der Arzt-Patienten-Beziehung zugunsten einer stärkeren Berücksichtigung der Patientenperspektive abzumildern und den Anspruch des Patienten, bei der Entscheidungsfindung mitzuwirken, verdeutlichen. Aus Sicht der Patienten selbst kann die ärztliche Entscheidung jedoch keineswegs durch eine Patientenverfügung ersetzt werden, sondern sie kann lediglich dazu dienen, die Patientenperspektive stärker mit einzubeziehen (ebd.). Patienten, die eine Patientenverfügungen verfasst haben, wollen laut Studienergebnissen nicht häufiger selbst entscheiden als andere Befragte, sondern sie haben vielmehr den Wunsch nach einem Arzt, der auf ihren „erhöhten Kommunikationsbedarf" eingeht (ebd. S. 198). Die Patienten, die dieser Gruppe angehören wollten zudem keineswegs einen Arzt, der sich ausschließlich in der Rolle eines Mediziners befindet, der die bereits formulierten Patientenwünsche erfüllt, sondern insbesondere mehr Zeit und mehr Gelegenheiten, mit dem Arzt die eigenen Situation zu besprechen (ebd.). Insgesamt scheint der Wunsch, eine Patientenverfügung verfassen zu wollen also vielmehr als eine Art initiatorisches Element für eine bessere Arzt-PatientenKommunikation zu dienen als ein tatsächlicher Zusatz für die ärztliche Entscheidungsfindung zu sein (vgl. Anselm 2008).

\subsection{Die Expertenautorität des Arztes}

Wie es im Verlauf der vorliegenden Arbeit deutlich wird, sind sowohl moralische Entscheidungen als auch ärztliche Handlungslogiken immer an ein gewisses Rollenbild gekoppelt, das Verhaltensweisen entweder legitimiert oder diese auf unterbewusste Art für die Handlungsakteure zum Teil unmöglich macht. Auch in allgemein gültigen ethischen Grundprinzipien findet sich dieser Aspekt wieder. Sowohl die Darstellung als auch die Reflexion ethischer Prinzipien sind implizit immer an Rollenverhältnisse gebunden. 
Um in der abschließenden Diskussion der Ergebnisse zu den genannten Aspekten eine begriffliche Klarheit zu gewährleisten, wird das folgende Kapitel einen Einblick in den soziologischen Diskurs zum Thema Profession und Expertenautorität des Arztes liefern.

Betrachtet man die professionssoziologische Literatur, so findet man beispielsweise bei Eliot Freidson, der Arztberuf fungiere mit seinem Ansehen in der Öffentlichkeit als eine Art Prototyp und könne exemplarisch gut dazu dienen, das Konzept der Profession zu analysieren und zu überprüfen (vgl. Freidson 1975). Im Allgemeinen beschreibt Freidson die Profession über zwei Gesichtspunkte. Zum einen definiert er sie als ein Aggregat von Menschen, die sich über eine gemeinsame Wertorientierung und gemeinsame Fähigkeiten definieren, die sich die Beteiligten über eine besondere Ausbildung oder ein Training angeeignet haben. $\mathrm{Ob}$ in diesem Zuge eine einzelne Person ein „Professional“ ist (Freidson 1975, S. 59) entscheidet man, indem man nach bestimmten internalisierten Werten fragt. Zum anderen wird die Profession als eine Gruppe Arbeitender betrachtet, die auf einer eher allgemeinen Ebene miteinander verbunden sind, nämlich in dem Sinne, dass sie in der Gesellschaft eine bestimmte gemeinsame Position inne haben und dass sie alle an einer bestimmten Arbeitsteilung beteiligt sind (ebd). Die einer Profession Angehörenden werden demnach in ihrem Verhalten danach beurteilt und definiert, wie sie ihr Arbeitsleben organisieren. All das geschieht unter der Grundannahme, dass sich ein Angehöriger einer bestimmten Profession aufgrund seines Status definieren lässt, ohne dass sich sein individuelles Verhalten in Hinsicht auf die Arbeitsstruktur, an der er beteiligt ist, erklären lässt (ebd.).

Betrachtet man im Allgemeinen die Analyse und Reflexion einer bestimmten Profession, findet man immer den Aspekt, wie sich diese in ihrer Beziehung zum Staat verhält. Freidson betont, dass insbesondere in der Medizin das staatlich garantierte Praxismonopol einen großen Teil der Stärke und das Ansehen der Profession ausmachen. Das Privileg und die Vormachtstellung, die ausschließlich dieser Profession erlaubt, beispielsweise Arzneimittel zu verschreiben, Laboruntersuchungen zu veranlassen oder Menschen in Krankenhäuser einzuweisen. Die ökonomische und politische Autonomie der medizinischen Profession ist in den unterschiedlichen Ländern unterschiedlich - in den USA beispielsweise wurde der Ärzteschaft durch die Berufsverbände weitgehend das Recht eingeräumt, zu bestimmen, wie für die Medizin relevante politische und gesetzliche Macht ausgeübt werden soll. Eine Gemeinsamkeit der Länder scheint ihre technologische und wissenschaftliche Autonomie zu sein, die sich dadurch auszeichnet, dass die Profession frei zu sein scheint, ihr spezielles Wissensgebiet weiter zu entwickeln und zu entscheiden, welches wissenschaftlich akzeptable und zu fördernde Praktiken sind (ebd.). 
Betrachtet man die medizinische Ausbildung, so ist bereits hier deutlich, dass der Medizinstudent durch den Inhalt und die Art des Studiums „sozialisiert wird, ein Arzt zu sein“ (Freidson 1975, S. 61) und dass er zu einem bestimmten Typ Mensch gemacht wird. Damals und auch heute noch stützt sich eine der elementaren Wertvorstellungen der ärztlichen Profession auf die traditionelle Vorstellung, dass ein Arzt das Leben eines Patienten in seiner Hand hat. Durch diese unmittelbar nahe Arbeit an ihren Patienten sind sie es jedoch auch, die Kritik und Tadel für Misserfolge einstecken (ebd.).

Ein weiterer interessanter Punkt, der die (mächtige) Position einer Profession unterstreicht, ist die Tatsache, dass eine Profession sich selbst kontrollieren muss (ebd.). Eine Analyse von Arbeit bedingt im klassischen Sinne stets eine Form der Leistungskontrolle, die jedoch hinsichtlich des Arztberufes nicht von außen, sondern lediglich durch sich selbst geschehen kann. Freidson sieht im Rahmen seiner Analyse zur Frage nach der Expertenautorität des Arztes die Beziehung zwischen Arzt und Patient an sich als problematisch an (ebd.). Verdeutlicht wird dieser Aspekt anhand der Tatsache, dass sich der Hilfesuchende mit einer für sich persönlich empfundenen Dringlichkeit an den Experten wendet, welche für diesen nicht mehr als eine Routineaufgabe darstellt. Stoßen Arzt und Patient in ihren Rollen als Berater und Klient aufeinander, hat jeder der beteiligten Personen eine andere Ansicht von dem (medizinischen) Problem und auch von der Problembewältigung dieser konkreten Gegebenheit. Neben den auf berufliche Erfahrung zurückzuführenden routinierten ärztlichen Handlungsweisen spielt bei den unterschiedlichen Perspektiven der Handlungsakteure auch das berufliche implizierte Spezialwissen des Arztes eine Rolle für die Sicht auf ein medizinisches Problem (ebd.). Freidson diskutiert an diesem Aspekt, dass ein berufsspezifisches Spezialwissen mit seiner jeweiligen Wirkung kennzeichnend für viele Professionen stehen kann, die Ärzteschaft jedoch über „die Behauptung, ein besonders esoterisches, wissenschaftliches oder abstraktes Wissen zu schulen, welches in markanter Weise der bloßen Erfahrung des Leidens an Krankheit oder dem Versuch, eine Reihe von an Krankheiten Leidenden pragmatisch zu heilen, überlegen ist.“ (ebd., S. 76). Aus diesem Grund geht in Hinsicht auf ein bestehendes Machtgefälle mehr in die Arzt-Patienten-Beziehung ein als nur eine unterschiedliche Perspektive, nämlich „die Differenz, in Bezug auf das, was dieser unterschiedlichen Perspektive unterworfen wird.“ (ebd., S. 76). Möglicherweise kommt ein Arzt in seiner Rolle als Experte zu einer Behandlungsstrategie, die dem Patienten weit entfernt vorkommt zu dem, was er selbst als körperliches Leid empfindet. Trotz der Tatsache, dass viele ärztliche Empfehlungen, die dem alltäglichen Wissen und dem Verstand des Patienten entgegenstehen, sind diese mittlerweile so üblich geworden, dass 
sie keine großen Probleme zwischen Arzt und Patent auslösen. Der Autor führt hierzu das Beispiel auf, bei Kopfschmerzen ein Medikament in den Magen zu geben. Hier muss der Patient sich in dieser Konstellation mit seinen eigenen Vorstellungen und seinem Vertrauen dem Arzt unterwerfen (vgl. Freidson 1975). Ein weiterer Aspekt, der das Machtgefälle zwischen Professionellen und Laien weiter beschreibt ist die Betrachtung, was Kompetenz an sich für eine Rolle spielt. Ein wesentlicher Aspekt, der besonders anhand von besonderes schwerwiegenden medizinischen Problemen deutlich wird, ist die Tatsache, dass Kompetenz stets mit einer Form von Machtbefugnissen einhergeht. Trotz des im modernen Medizinsystem deutlichen Argumentationsfokus auf die Autonomie des Patienten sind Machtstrukturen in der Arzt-Patienten-Interaktion stets greifbar.

Neben den beschriebenen Aspekten hat Parson die Rolle der speziellen technischen Kompetenz in der Medizin genauer analysiert und dessen starke Autoritätsfunktion dargelegt (vgl. Parson 1947). Während sich nach Parson Autorität dadurch kennzeichnet lässt, dass sich über sie Sanktionen erteilen und legitimieren lassen, fehlt dieser Aspekt der speziellen technischen Autorität der Medizin. Die Art und Weise Patienten dazu zu bringen, sich beraten zu lassen, zu vertrauen und am Ende sogar zu gehorchen ist laut des Autors also deutlich abstrakter als in anderen Berufen (ebd.). Dennoch bleibt das Problem, das sich mit professioneller Autorität gegenüber Laien ergibt, bestehen. Dem Laien fehlt die bildungsmäßige und die erfahrungsmäßige Vorraussetzung, die es ihm ermöglichen würden, aus einer professionellen Sicht und aus professionellen Gründen heraus zu entscheiden, ob ein bestimmter Ratschlag zu befürworten und anzunehmen ist oder nicht. Freidson betont anhand dieses Aspekts die zu kritisierende Ausgangslage des Professionellen, der seinen Klienten bis zum Zustimmen, bzw. zum Gehorsam überzeugen muss (vgl. Freidson 1975). Ein anderes Problem, dass laut des Autors in jeder Interaktion zwischen Laien und Professionellen eine Rolle spielt ist, dass Experten stets auf die Kooperation der Laien angewiesen sind, die jedoch in den meisten Fällen weder die „berufseigenen Beweisregeln“ (ebd., S. 79) noch die inhaltlichen Grundlagen der Expertenfähigkeit kennen.

Was den Angehörigen einer Profession von allen anderen beratenden Experten unterscheidet, ist die Fähigkeit, einige dieser Probleme der Autorität durch formelle, institutionelle Vorkehrungen zu lösen. Seine Lösung vermindert in der Interaktion mit seiner Klientel die Rolle der überzeugenden Beweisführung. (Freidson 1975, S. 79). 
Die hier zitierte Form der institutionalisierten Problemlösung übt also eine besondere Form der Autorität aus, die die Rolle einer Profession und die zu ihr gehörenden Personen deutlich beschreibt.

Ein weiterer von Freidson herausgearbeiteter Aspekt, der die Autorität und das Ansehen des Arztes in der Gesellschaft untermauert, ist die Tatsache, dass praktizierende Mediziner ganz im Gegensatz zu Wissenschaftern fast ausschließlich mit Laien in Kontakt sind (ebd.). Die Arbeitsweisen und die Fähigkeiten eines Wissenschaftlers bleiben im Kontrast dazu für die Öffentlichkeit dauerhaft wenig fassbar, da diese nur in ihrem professionellen Kollegenumfeld arbeiten.

Betrachtet man die Autorität und das Ansehen einer Profession an sich an dieser Stelle noch einmal genauer, so findet sich im soziologischen Diskurs wiederholt der Aspekt, dass das Ansehen einer Profession viel mehr durch eine Form von Autonomie als durch Qualitäten, wie Intelligenz, ethische Einstellung und erlernte Fähigkeiten bestimmt wird. Freidson beispielsweise arbeitet mit einem Autonomiebegriff, der für „die Eigenschaft oder den Zustand, unabhängig, frei, zur Selbstbestimmung fähig zu sein“ steht (ebd., S. 95). Kernelement dieses Aspekts ist also die Tatsache, dass sich Angehörige einer Profession selber leiten und kontrollieren. Freidson leitet anhand dieses Phänomens die Kernpunkte aller weiteren Definitionen ab, die eine Profession und ihre Rolle ausmachen. So formuliert er, dass beispielsweise dann eine höhere Wahrscheinlichkeit der Selbstbestimmung besteht, wenn eine Berufsgruppe „zu einer legalen oder politischen Vorzugsstellung gekommen ist, die sie vor der Einmischung von Seiten anderer Berufe schützt“ (ebd., S. 95). Genau dieser Aspekt ist charakteristisch für die ärztliche Profession. Der Ärzteschaft allein ist es, wie bereits erwähnt, vorbehalten, Medikamente zu verschreiben und den menschlichen Körper beispielsweise operativ zu behandeln. Ein weiterer Punkt ist die Art der Lehre, die bis heute nach außen hin klar den Eindruck erweckt, eine Art exklusives Wissen und einen theoretischen Inhalt zu lehren, welcher keineswegs durch andere universitäre Fakultäten zu vermitteln sei. Eine weitere für die ärztliche Profession typische Methode, anderen zu erklären, man könne ihrer Profession vertrauen, ist ein gewisser interner ethischer Kodex, der sich grundsätzlich auf die Verfolgung rein guter Absichten beruft (ebd.). Ein Element, welches quasi als Methode dafür fungiert, die Gesellschaft dazu zu bringen, der ärztlichen Profession ihren autonomen Status zu legitimieren und ihn gleichermaßen zu billigen. So formuliert Freidson, dass „die bloße Existenz eines solchen ethischen Kodex impliziert, dass die einzelnen Mitglieder des Berufs sämtlich mit den personalen Qualitäten des Professionalismus begabt sind [...].“ (Freidson 1975, S. 96). 
Interessant ist, dass die meisten erwähnten Eigenschaften der Profession sowohl als Folgen ihrer Autonomie als auch als Bedingungen zu lesen sind, die offensichtlich benötigt werden, um die Öffentlichkeit und die wichtigen politischen Instanzen zur Gewährung dieser Autonomie zu bringen (ebd.).

Ein letzter Aspekt des soziologischen Diskurses, der jedoch im Zuge der Etablierung des informed constent heutzutage weniger schwer ins Gewicht fält, ist die Rolle, in die Patienten zum Teil gedrängt werden. Lange herrschte die professionelle Ansicht, die Fülle und die Komplexität einer medizinischen Information würde den kranken Patienten überfordern und Schwierigkeiten in der Problembewältigung mit sich bringen (ebd.). „Folglich sollte der Patient nicht wie ein Erwachsener behandelt werden; man sollte ihm eher wie einem Kind Beruhigung, aber nicht Informationen zuteil werden lassen." (ebd., S. 101). Auch wenn sich die Aussage heute in dieser Form sicher nicht rezipieren ließe, setzt ein nicht unerheblicher Teil der Ärzteschaft bei der Darlegung ihrer medizinischen Vorhaben eher auf Vertrauen als auf Überzeugung mittels ausführlicher Aufklärung. Wird also auch in einer modernen Arzt-Patienten-Beziehung auf Vertrauen oder sogar Zutrauen bestanden, so folgt nach Freidson daraus, dass im gleichen Zuge auch darauf bestanden wird, dass der Patient seine Rolle als unabhängiger Erwachsener aufgibt (vgl. Freidson 1975). Indem man Patienten in die beschriebene Rolle befördert, wird parallel dazu die Autorität und die eindeutig starke Rolle des Arztes geschützt. 


\section{Zwischenfazit}

Unter dem Titel Autonomie zwischen Ideal und Realität - Eine empirisch-ethische Reflexion eines Prinzips anhand ärztlicher Kommunikationsstrategien am Lebensende nimmt das vorliegende Forschungsanliegen Bezug auf ein normatives Prinzip der Medizinethik. Die Realisierbarkeit des Autonomieprinzips in der medizinischen Praxis ist indes das Kernanliegen der Arbeit.

Bevor im Folgenden das methodische Vorgehen erläutert wird, soll an dieser Stelle erneut auf die Intention der Arbeit auf der Basis des theoretischen Hintergrundes eingegangen werden.

Vor der Praxisreflexion wurde zunächst der Fachbereich Palliativmedizin als ein potentielles Modell für die praktische Anwendung des Autonomieprinzips betrachtet. Der verhältnismäßig junge Fachbereich ist Kernelement einer Dynamik, die im Rahmen ihres vielschichtigen Behandlungskonzepts den aktuell zentralen Bezugspunkt des Argumentierens der Medizinethik auf verschiedenen Ebenen starkmacht. Mit ihrem klaren Fokus auf den Erhalt und die Förderung von Patientenautonomie übernimmt sie dadurch eine Art Vorreiterfunktion. Es scheint demnach hinsichtlich einer Diskursgrundlage wertvoll zu sein, bei der Frage nach der Realisierbarkeit des Autonomieprinzips die Palliativmedizin mit ihren Idealen in den Vordergrund rücken zu lassen.

Nach der Betrachtung der Palliativmedizin mit ihrem Bezug zu Autonomie erfolgte die Aufarbeitung des Autonomieprinzips aus medizinethischer Perspektive. Anhand der einschlägigen Literatur wurde analysiert, wie sich das Prinzip der Autonomie im Diskurs der Bioethik entwickelt hat. Grundsätzlich speist sich der Diskurs aus der klassisch philosophischen Debatte. Im Rahmen modernerer Ansätze werden die klassischen Elemente zwar aufgegriffen, sie setzen dem jedoch die Beziehungshaftigkeit des Menschen deutlich gegenüber.

In dem empirischen Teil des vorliegende Forschungsvorhabens werden vor dem Hintergrund der Frage nach dem Verwirklichungshorizont von Autonomie beide Aspekte berücksichtigt.

Im Detail zeigen die unterschiedlichen theoretischen Zugänge zu Autonomie deutlich, dass das Prinzip der Autonomie nicht als absolut oder im Sinne eines universal gültigen Prinzips gesehen werden kann. Autonomie wird als relative Größe beziehungsweise als gradueller Begriff verstanden, der stets soziale Beziehungen und Strukturen mit einbezieht. Die Autonomie des Einzelnen steht dabei nicht im Gegensatz zu Beziehungen, sondern es wird stets beides zusammen gedacht. Wesentlicher Gedanke ist dabei die Distanzierung 
von absolut gedachter Autonomie hin zu einer Orientierung an der lebensweltlichen Praxis. Dabei ist Autonomie nicht einfach gegeben oder nicht gegeben, sondern stets in unterschiedlichem Ausmaß existent. Zudem ist die Autonomie des Einzelnen abhängig von der konkreten Situation und in konkrete Handlungskontexte eingebunden. In den theoretischen Ausarbeitungen der Medizinethik lassen sich die genannten Aspekte unter dem Begriff der relationalen Autonomie zusammen fassen. Dabei kann festgehalten werden, dass relationale Autonomie mit der Anerkennung von gegenseitiger Interdependenz einhergeht. Autonomie kann in Zusammenschau der unterschiedlichen theoretischen Ausarbeitungen nur in zwischenmenschlichen Beziehungen gelebt und verwirklicht werden.

Folgt man diesem theoretischen Aspekt muss die Betrachtung von Beziehungen bei der Frage nach der Realisierbarkeit des Autonomieprinzips ein wesentliches Element sein. Um sich dem Aspekt der (Arzt-Patienten)-Beziehung annähern zu können, fokussiert die vorliegende Arbeit sowohl in ihrer grundlegenden Intention als auch im methodischen Vorgehen den Aspekt der Kommunikation. Als bedeutsamer Bestandteil zwischenmenschlicher Beziehungen kann Kommunikation für sich allein stehend bereits als Autonomie förderndes Instrument betrachtet werden. Im Rahmen des Forschungsanliegens wird die Untersuchung der Kommunikationsstrategien praktizierender Mediziner sowohl für sich stehend betrachtet als auch im Sinne eines Werkzeugs der Praxis für die Analysen der Arbeit nutzbar gemacht. Dieser Aspekt spiegelt sich auch in dem gewählten methodischen Vorgehen, der qualitativen Interviewstudie, wider.

Zentral für die Diskussion um das Prinzip der Autonomie ist die parallel dazu geführte Debatte zum Würdebegriff. Es erfolgte somit über die bisher erwähnten theoretischen Ausarbeitungen hinaus eine theoretische Darstellung des Begriffs. Die grundlegenden philosophischen Ansätze sowie die in der Literatur diskutierten Schwierigkeiten, die mit dem Begriff der Würde einhergehen, untermauern dabei einen wesentlichen Aspekt des vorliegenden Forschungsanliegens. Sowohl für die Akteure des medizinischen Praxisgeschehens als auch für Wissenschaftler, die in ihren Disziplinen Handlungsanweisungen für die medizinische Praxis formulieren, ist es unabdingbar, reflektiert mit Begriffen, wie dem Würde- oder Autonomiebegriff umzugehen. Ist dies nicht der Fall, so zeigen exemplarische Situationen der Praxis, dass neben der Tatsache, dass Kommunikation an sich bereits Autonomie fördernd sein kann, eine fehlende Auseinandersetzung mit den eigenen Kommunikationsformen dazu führen kann, dass Handlungsspielräume und damit die Autonomie des Patienten eingeschränkt werden 
können. Dies zeigt sich insbesondere im Umgang mit Menschen, die aufgrund einer infausten Prognose eine Verschiebung ihres vorherigen Wertehorizonts erfahren oder aufgrund des biographischen Bruchs durch eine Erkrankung Selbstbestimmung vollkommen verändert wahrnehmen. Wenngleich die Entwicklung unstrittig ist, das Prinzip der Autonomie im traditionell sehr hierarchisch geprägten Medizinsystem in den Vordergrund rücken zu lassen, braucht es für die verschiedenen Akteure der Praxis mehr als ein theoretisches Ideal. In welcher Weise das Prinzip der Autonomie für das praktische Medizinsystem Bedeutung beanspruchen kann, lässt sich nicht allein durch eine theoretische Analyse beantworten. Für die Fragestellung bedarf es gleichermaßen einer Analyse des faktischen Einsatzes im innerkommunikativen Kontext.

Einen Beitrag zu diesem Kontext soll das vorliegende Forschungsanliegen mit seinem konkreten Blick in die Praxis leisten. Um im Rahmen dessen eine Annäherung an die oftmals fehlende Verzahnung zwischen Theorie und Praxis zu erzielen, spielt sich das vorliegende Forschungsanliegen auf zwei unterschiedlichen Ebenen ab. Angesichts dessen ist es notwendig sowohl einem operationalen als auch einem ethischen Problemzugriff $z u$ folgen. Der ethische Zugriff beinhaltet das Autonomieprinzip als normatives Prinzip der Medizinethik und die Ausarbeitungen zu dem Begriff der Würde. Der operationale Zugang stell mit seinem Fokus auf Kommunikationsstrategien den Praxisbezug her und begründet das methodische Vorgehen des Forschungsvorhabens. Darüber hinaus verknüpft die vorliegenden Arbeit normative Elemente der Medizinethik und deskriptive Elemente empirischer Forschung. Die Intention, eine Verzahnung zwischen Theorie und Praxis herzustellen, wird dadurch gewährleistet.

Fokus der Arbeit ist die Frage der Realisierbarkeit des Autonomieprinzips in der medizinischen Praxis. Die Analyse soll dabei nicht primär mit einem weiteren Kritikpunkt einhergehen, sondern einen konstruktiven Blick in die Praxis mit sich bringen. Es soll beleuchtet werden, inwiefern das Autonomieprinzip, wie es als Ideal in der Palliativmedizin theoretisch entworfen wird, sich aus der Perspektive von Palliativmedizinern und kurativ tätigen Medizinern in deren Handlungswirklichkeit darstellt. Eine zentrale Analysegrundlage bildet dabei der Autonomiediskurs in der Medizinethik. Da sich, wie bereits erwähnt, theoretischer Diskurs und komplexes Praxisgeschehen häufig wenig berühren soll insbesondere untersucht werden, inwiefern das theoretische Konzept der Autonomie in der Palliativmedizin für die Praxis handlungsrelevant wird und wie sich im Umgang damit alternative Überlegungen der medizinethischen Debatte als hilfreich erweisen können. Die Analyse soll in erster Linie anhand der erzählten Wahrnehmung von Medizinern erfolgen. Dabei werden deren Kommunikationsstrategien und ihr Einfluss auf die Autonomie des 
Patienten untersucht. Zudem soll beleuchtet werden, ob auch eine Überbetonung von Autonomie selbst dazu führen kann, dass Konflikte entstehen oder dass Handlungsspielräume von Patienten potentiell eingeschränkt werden. Bezogen auf die Bedeutung des Autonomiebegriffs für die Praxis schränken die vorliegenden Überlegungen dessen Wert nicht ein, sondern es soll vielmehr verdeutlicht werden wie notwendig eine Auseinandersetzung mit dem Begriff und seinem Prinzipiencharakter für eine reflektierte Praxis ist.

Durch die Verknüpfung zwischen den normativen Anteilen der Medizinethik und der deskriptiven empirischen Forschung andererseits soll der Diskurs durch die vorliegende Arbeit mit ihrer Praxisnähe bereichert werden. 


\section{Methoden}

\subsection{Empirisch-qualitative Forschung anhand von Experteninterviews}

Für die Untersuchung der Fragestellung, welche Rolle ärztliche Kommunikationsstrategien für die Realisierbarkeit des Autonomieprinzips spielen, wurde die sozialwissenschaftliche Methodik der qualitativen Interviewstudie gewählt. Wie bereits erläutert wurde untersucht, inwiefern das Autonomieprinzip, wie es als theoretisches Idealbild entworfen wurde, sich aus der Perspektive von Palliativmedizinern und kurativ tätigen Medizinern in deren Handlungswirklichkeit darstellt.

Im Konkreten bildet das Leitfaden gestützte Experteninterview den methodischen Hintergrund des vorliegenden Forschungsvorhabens. Das Experteninterview dient der Rekonstruktion komplexer Wissensbestände, die für die Erklärung sozialer Phänomene relevant sind (vgl. Liebold und Trinczek 2009). Leitfadeninterviews sind stets thematisch strukturiert. Ihr Ziel ist es, die Selbstdarstellung der Interviewpartner durch erzählgenerierende Fragen zu motivieren. Der im Voraus entwickelte strukturierte Interviewleitfaden ist dabei so konzipiert, dass genügend Raum für freie Erzählpassagen mit eigenen Relevanzsetzungen der Interviewpartner bleibt (vgl. Hopf 2003). Im Rahmen des Leitfadeninterviews ist es möglich, die Abfolge und Form der Fragen entsprechend flexibel handzuhaben.

Wie einleitend erwähnt geht die argumentative Fokussierung auf das Prinzip der Autonomie und die damit einhergehende Wahrnehmung des Prinzips als einer Art Korrektiv auch mit Kritik einher. Aus den kritischen Ausführungen, die eine problembezogene Betrachtung des Autonomiebegriffs gemeinsam haben, sind daraufhin verschiedenen Konzepte entstanden, welche sich unter dem Begriff der relationalen Autonomie zusammenfassen lassen. Um innerhalb des komplexen medizinischen Praxisgeschehens zu analysieren, inwiefern derartige Konzepte handlungsrelevant werden können, bedarf es somit einer Methode, die über eine Hypothesenüberprüfung hinausgeht und den Interviewpartnern Raum zur Entfaltung ihrer eigenen Relevanzsetzungen ermöglicht. Da das Experteninterview so konzipiert ist, dass genau diese genannten Aspekte berücksichtigt werden, bildet es die methodische Grundlage des vorliegenden Forschungsvorhabens.

Im Kontrast zu den sogenannten hypothesenprüfenden Verfahren zeichnet sich das Experteninterview dadurch aus, dass man zur Rekonstruktion der Erfahrungswirklichkeit empirisch an der Alltagswelt der Interviewpartner ansetzt. Bei den hypothesenprüfenden Verfahren ist diese eine von den Subjekten unabhängige im Vorfeld formulierte 
Hypothese. Im Rahmen des Experteninterviews wird konsequent versucht die in der Alltagswelt der Subjekte generierten Erfahrungen, Perspektiven und Relevanzstrukturen zu rekonstruieren (vgl. Liebold und Trinczek 2009). Der Interviewte wird dabei nicht im Sinne eines individuell agierenden Experten gesehen, sondern als „Repräsentant einer Gruppe“ (Galuschko und Voltz 2011, S. 54). Durch die weitgehende Eigenpräsentation der Interviewpartner wird ein Oktroyieren künstlicher Aspekte weitgehend vermieden und ein notwendiges $\mathrm{Maß}$ an Offenheit erreicht. Die Datengewinnung vollzieht sich dabei als kommunikativer Prozess. Ein weiteres Kennzeichen ist die schrittweise Gewinnung und Prüfung von Daten. Dabei entwickeln sich der Zusammenhang und die Beschaffenheit der einzelnen Elemente allmählich und in einem ständigen reflexiven Bezug (vgl. Witzel 2000). Es ergibt sich im Rahmen des Forschungsprozesses also unter Umständen eine andere Dimension der Wirklichkeitskonstruktion als es die Wissenschaftler vorher erwarten. In Zusammenschau seiner Charakteristika eignet sich das Experteninterview demnach zur Exploration des Unbekannten.

Die Methodik der qualitativen Interviewstudie bringt jedoch auch Limitationen mit sich. Hopf zufolge ist dies beispielsweise die Tendenz zu einem dominierenden Kommunikationsstil. Zudem ergeben sich laut des Autors Probleme mit passiv-rezeptiven Anteilen des Interviewens und eine aus Unsicherheit resultierende Unfreiheit des Interviewers im Umgang mit dem Interviewleitfaden (vgl. Hopf 2003). Die Tendenz zu einem dominierenden Kommunikationsstil kann zu einer Häufung von suggestiven Fragen führen. Außerdem können bewertende und kommentierende Aussagen seitens des Interviewers vorkommen, die das Interview und bestimmte Überlegungen ungewollt lenken. Eine bestehende Unsicherheit im Umgang mit dem im Voraus erstellten Leitfaden kann außerdem zum Verlust des Erkennens interessanter und nicht antizipierter Themen führen. Außerdem findet über die Bedeutung des sogenannten Expertentums ein kritischer Austausch statt (vgl. Bührmann 2004).

Die vorliegende Arbeit berücksichtigt diese Punkte dahingehend, dass sehr frei mit dem Interviewleitfaden gearbeitet wurde. In dem Fall, dass einer der interviewten Experten ein bestimmtes Themengebiet des Leitfadens oder darüber hinaus ansprach, bevor es im Interview vorgesehen war, wurde dies berücksichtigt und zunächst darauf eingegangen. Dabei fungierte der Interviewleitfaden mehr als Orientierung und legte die Abfolge der Fragen nicht fest. Wie von Hopf für das Experteninterview gefordert, wurde zudem das aktive Fragen und Nachfragen des Interviewers sowie sein vorsichtiges Argumentieren und Aufbauen von möglichen Gegenpositionen berücksichtigt (vgl. Hopf 2003). Die durchgeführten Interviews wurden somit als flexible teilstandardisierte, bzw. halb 
strukturierte Interviews geführt (vgl. Mayring 2010). Zudem wurde das erste Interview als Probeinterview durchgeführt und hinsichtlich der genannten Aspekte sowie hinsichtlich der Justierung des Fragebogens gemeinsam mit erfahrenen Sozialwissenschaftlern analysiert.

\subsection{Erstellung und orientierender Inhalt des Interviewleitfadens}

Im Vorfeld erfolgte zunächst eine Einarbeitung in den medizinethischen Diskurs zu dem Themenkomplex Autonomie, sowie in die palliativmedizinische Grundlagenliteratur. In einem nächsten Schritt folgte im Rahmen von Vorgesprächen und eines Probeinterviews eine Sondierung der für das Forschungsvorhaben relevanten Themengebiete.

Ziel war es, die Erfahrungswirklichkeit der Interviewpartner rekonstruieren zu können und nicht nur abstrahiert über Kommunikationsformen und über deren Wirkung auf die Autonomie des Patienten zu sprechen. Der im Rahmen mehrerer Arbeitsschritte entwickelte Interviewleitfaden wurde so erstellt, dass über die zunächst erfolgte Deskription der konkreten Arzt-Patienten-Interaktion hinaus eine Reflexion über die eigenen Kommunikationsformen und deren Wirkung stattfinden konnte. Um den Themenkomplex Fragen am Lebensende greifbar zu machen wurde nach dem konkreten Umgang mit Aufklärungsgesprächen über eine Erkrankung mit einer infausten Prognose gefragt. Auf den dargestellten Szenarien aus der Praxis aufbauend wurde explizit nach Kommunikationsformen, nach ausgewählten Vokabeln und nach der Selbstreflexion bezüglich der Wahrnehmung und Förderung von Patientenautonomie gefragt. Darüber hinaus erfolgte in jedem Schritt gemeinsam mit den Interviewpartnern eine Reflexion hinsichtlich der Realisierbarkeit von Patientenautonomie im medizinischen Praxisgeschehen. (Interviewleitfaden im Anhang).

\subsection{Auswahl des Samples und Durchführung der Interviewstudie}

Für die vorliegende Studie wurden insgesamt 21 Leitfadeninterviews in ganz Deutschland geführt. Die Interviews wurden von der Autorin Lena Woydack mit der Unterstützung von Frau Dr. Julia Inthorn und Frau Prof. Dr. Silke Schicktanz zwischen März 2012 und August 2012 durchgeführt. Die Teilnahme an den Interviews war freiwillig. Alle 21 angefragten Personen sagten zu. Um eine möglichst heterogene Gruppe für die Gewinnung des empirisches Materials zu gewinnen, wurden die Interviewpartner sowohl im ländlichen Raum als auch im Raum großer (Universitäts-)Kliniken rekrutiert. Mit Blick auf den Themenschwerpunkt erschien es sinnvoll, primär Ärzte mit längerer Berufserfahrung zu 
befragen, so liegt der Altersdurchschnitt etwa bei 50 Jahren. In dem Sample finden sich etwas mehr Männer als Frauen, was nicht beabsichtigt war, jedoch der üblichen Geschlechterverteilung dieser Altersgruppe in dem befragten Berufsbild entspricht.

Tabellarische Darstellung der Interviewteilnehmer6:

\begin{tabular}{|l|l|c|c|c|c|}
\hline & Fachbereich & Erfahrung & PM & I & S vs. L \\
\hline M, 58 & Allgemeinmedizin & 20 & - & + & L \\
\hline M, 57 & Geriatrie & 10 & - & + & S \\
\hline M, 42 & Palliativmedizin & 7 & + & + & S \\
\hline M, 50 & Allgemeinmedizin & 5 & - & + & S \\
\hline W, 51 & Gastroenterologie & 20 & + & + & S \\
\hline M, 58 & Gastroenterologie & 22 & - & + & S \\
\hline W, 43 & Gastroenterologie & 11 & - & - & S \\
\hline W, 39 & Palliativmedizin & 7 & + & + & L \\
\hline M, 45 & Onkologie & 12 & + & + & S \\
\hline M, 38 & Palliativmedizin & 1 & + & + & S \\
\hline M, 63 & Palliativmedizin & 20 & + & + & L \\
\hline M, 45 & Palliativmedizin & 7 & + & + & S \\
\hline M, 50 & Strahlentherapie & 8 & - & + & S \\
\hline M, 51 & Allgemeinmedizin & 9 & + & - & S/L \\
\hline W, 39 & Palliativmedizin & 6 & + & + & S \\
\hline M, 48 & Onkologie & 15 & + & + & S \\
\hline W, 45 & Palliativmedizin & 5 & + & - & L \\
\hline M, 46 & Palliativmedizin & 7 & + & - & S/L \\
\hline M, 39 & Palliativmedizin & 5 & + & + & S \\
\hline M, 55 & Palliativmedizin & 11 & + & + & S \\
\hline W, 52 & Palliativmedizin & 10 & + & + & S \\
\hline
\end{tabular}

${ }^{6}$ Erfahrung: Jahre in aktuellem Beruf; PM: Weiterbildung Palliativmedizin; I: interdisziplinär tätig; S vs. L: Arbeit in städtischer Klinik (S) oder auf dem Land (L) 
Zum Zeitpunkt der Befragung waren elf Interviewpartner in der Palliativmedizin tätig und zehn arbeiteten derzeit in einem kurativen Fachbereich. Die ausgewählten kurativen Bereiche wiesen mit Blick auf das Diagnosespektrum und die in diesem Zusammenhang notwendigen Gespräche über mögliche infauste Prognosen Berührungspunkte zu den Gesprächssituationen der Palliativmedizin auf und brachten somit eine gute Möglichkeit des Vergleiches mit sich.

\subsection{Datenerfassung und Transkription}

Die Datenerhebung erfolgte bei allen 21 Interviews durch die Autorin persönlich und fand an den von den Interviewpartnern ausgewählten Orten statt. Dies waren in der Regel Arztoder Besprechungszimmer der jeweiligen Klinik oder Praxis. Bis auf wenige Ausnahmen hatten sich die Interviewpartner alle einen gesonderten Termin für das Interview eingeräumt, sodass es zu keinen nennenswerten Störungen oder besonderen Vorkommnissen kam. Die Interviews dauerten zwischen 45 und 100 Minuten und wurden mit der Zustimmung der Teilnehmer mit einem Aufnahmegerät aufgezeichnet. Die Audiodateien, die im Rahmen der Interviews entstanden, wurden anonym gespeichert und anschließend vollständig transkribiert. Es wurde bei der Transkription der Interviews darauf geachtet, dass alle gesprochenen Informationen vollständig pseudonymisiert wurden. Prinzipiell sind verschiedene Arten der Verschriftlichung möglich: Standardorthographie, literarische Umschrift, eye dialect und phonetische Umschrift?. Die Transkription der durchgeführten Interviews erfolgte aufgrund der erleichterten Lesbarkeit anhand der Standardorthographie. Diese orientiert sich an der schriftlichen Sprache und macht das Transkribieren einfach und effizient. Besonderheiten der Sprache, wie das Auslassen von Lauten oder die Angleichung aufeinander folgender Laute werden hierbei vernachlässigt. Es wurden demnach ausschließlich Merkmale des Gesprächsverhaltens transkribiert, die auch tatsächlich analysiert wurden (vgl. O'Connell und Kowal 2003).

\footnotetext{
${ }^{7}$ Die Verschriftlichung berücksichtigt sprachliche Abweichungen wie etwa in der Elision von „e “ in „gehn“ für „gehen“ oder in der Assimilation „haste“ für „hast du“. Durch den eye dialect soll die Umgangssprache möglichst lautgetreu abgebildet werden, wie etwa bei „askche“ für "asked you“. (vgl. O'Connell und Kowal 2003).
} 
Transkriptionsempfehlungen gemäß O’Connell und Kowal (vgl. O’Connell und Kowal 2003)

Es sollten nur solche Merkmale des Gesprächsverhaltens transkribiert werden, die auch tatsächlich analysiert werden.

Um die Eindeutigkeit von Notationszeichen zu sichern, sollten Buchstaben für die Darstellung verbaler Merkmale von Äußerungen und Interpunktionszeichen nur in ihrer gebräuchlichen Funktion verwendet werden.

Subjektive Wahrnehmungen und/ oder Kategorisierungen von Transkribierenden sollten nicht als objektive Messung notiert werden.

In einem Transkript soll deutlich erkennbar zwischen Beschreibungen, Erklärungen, Anmerkungen und Interpretationen unterschieden werden.

Bei der Analyse von Transkripten soll berücksichtigt werden, dass Transkribierende als Sprachnutzer nicht selten unzuverlässig transkribieren. Daher müssen die Audiodaten für einen bestimmten Zeitraum als Sicherheit und zur Wiederprüfung erhalten bleiben.

\subsection{Datenstrukturierung und Datenanalyse}

Die für die Analyse entwickelten Auswertungskategorien entstanden in der intensiven Auseinandersetzung mit dem gewonnenen empirischen Material, welches auf der Grundlage des aus der ethischen und medizinischen Fachdebatte konstruierten Interviewleitfadens gewonnen wurde. Dem Entwurf von bestehenden Themenkatalogen wurde im Rahmen dessen soweit Rechnung getragen, als dass Kodierungen nicht ausschließlich frei anhand des Materials entwickelt, sondern gleichzeitig unter Berücksichtigung des zugrunde liegenden Leitfadens erarbeitet wurden. Die methodologischen Prinzipien, die ein systematisches Vollziehen der Analyseschritte der qualitativen Forschung zur Verhinderung von vorschnellen Quantifizierungen und Bewertungen verlangen, wurden dabei durchgehend beachtet (vgl. Mayring 2010).

Die Auswertungsstrategie, die bei der Analyse der Interviews verfolgt wurde, enthält folgende Arbeitsschritte: Zunächst wurden in der Auseinandersetzung mit dem Material sogenannte Kodierungen für die Strukturierung und Auswertung gebildet (Codes 1. Ordnung). Diese wurden in einem zweiten Schritt anhand des Materials erprobt, überarbeitet und verfeinert (Codes 2. Ordnung). Mit Hilfe der Codes 2. Ordnung wurde im Verlauf das gesamte Material kodiert. Auf Grundlage der Kodierung wurden dann im nächsten Schritt Übersichten erstellt. Diese fungierten im abschließenden Auswertungsschritt als Grundlage zur Auswahl einzelner Fälle für vertiefende Einzelfallanalysen. Für die Entwicklung der Auswertungskategorien wurde das empirische 
Material intensiv und wiederholt durchgelesen, kodiert und im Verlauf reduziert. Im Rahmen dessen wurde computerunterstützt gearbeitet (Atlas.ti).

Im ersten Arbeitsschritt wurden die verschiedenen Interviews noch nicht vergleichend, sondern jeweils für sich sprechend betrachtet. Die das einzelne Interview prägenden Themen wurden notiert. Für die folgenden Auswertungsschritte wurden Ähnlichkeiten und Unterschiede jedoch bereits festgehalten. Parallel dazu wurden, basierend auf der Grundlage des Interviewleitfadens und der in den Interviews aufgeworfenen Themen, Kodierungen in Form der Codes 1. Ordnung formuliert. Es wurden Definitionen für die verschiedenen Codes 1. Ordnung erstellt, die eine eindeutige Zuordnung des Interviewmaterials bei dem Kodieren mit den Codes 1. Ordnung ermöglichen sollten. Darüber hinaus wurden während des Kodierens Beispielpassagen aus dem Interviewmaterial entnommen und den jeweiligen Codes 1. Ordnung zugeordnet, um eine fortgehende Orientierung beim Kodieren zu erlauben. Neben der Entwicklung und Definition der Codes und der Nutzung von Beispielpassagen wurde außerdem ein Kodierleitfaden erstellt. In diesem wurden Regeln formuliert, anhand derer Probleme der Abgrenzung zwischen verschiedenen Codes begegnet wurden.

Die folgende Tabelle zeigt die Codes 1. Ordnung, welche nach mehrmaligem Lesen des Materials in Zusammenhang mit dem Interviewleitfaden erstellt und auf das Interviewmaterial angewendet wurden.

Codes 1. Ordnung

\begin{tabular}{|l|}
\hline Autonomie \\
\hline Arzt-Patienten-Beziehung \\
\hline Kommunikationsstrategien \\
\hline Ideale der Palliativmedizin \\
\hline Würde \\
\hline Sterben und Tod \\
\hline Aufklärung und Authentizität \\
\hline
\end{tabular}

Im darauffolgenden Arbeitsschritt wurden inhaltlich vertiefende Kodierungen erarbeitet (Codes 2. Ordnung), die bei der weiteren Sichtung des Materials eine feinere und detailreichere Kodierung ermöglichten. Dadurch wurde die Arbeit, wie von Mayring beschrieben, entscheidend strukturiert und präzisiert (Mayring 2010). Anhand der Codes 
2. Ordnung wurden alle Interviews einzeln mit Hilfe des Computerprogramms Atlas.ti kodiert. Die Codes 2. Ordnung wurden in diesem Schritt auf das Material selbst angewandt. Zudem wurden auch für die Codes 2. Ordnung Definitionen erstellt und Beispielpassagen aus dem Interviewmaterial entnommen, die den jeweiligen Codes 2. Ordnung zugeordnet wurden, um eine fortgehende Orientierung beim Kodieren zu ermöglichen. Darüber hinaus wurde der Kodierleitfaden auf dem Boden der Codes 2. Ordnung angepasst und präzisiert. Sowohl die Codes 1. Ordnung als auch die Codes 2. Ordnung, die durch eine Spezifizierung und Verfeinerung der Codes 1. Ordnung geprägt sind, haben dabei einen deduktiven sowie einen induktiven Charakter.

\section{Codes 2. Ordnung}

\begin{tabular}{|l|}
\hline Autonomie als anerkanntes Ideal \\
\hline Spannungen zwischen Theorie und Praxis im Umgang mit Autonomie \\
\hline Medizinische Indikation als Grenze von Patientenautonomie \\
\hline Angst vor Autonomieverlust \\
\hline Einschränkung von Autonomie \\
\hline Autonomie des Patienten entgegen ärztlicher Verantwortung \\
\hline Ambivalenz von Autonomie \\
\hline Ganzheitliche Persönlichkeitswahrnehmung \\
\hline Demut im Umgang mit der eigenen Machtposition \\
\hline Imperativ der Eigenverantwortung des Arztes \\
\hline Rollenkonsistenz \\
\hline Machtbereich des Arztes \\
\hline Arzt-Patienten-Interaktion \\
\hline Authentizität \\
\hline Verdrängung \\
\hline Das Idealbild des Arzt-Patienten-Gesprächs \\
\hline Resonanz des Patienten als Variable \\
\hline Wahrnehmung des Subtextes des Patienten \\
\hline Raum für Kommunikation über Sterben und Tod \\
\hline
\end{tabular}




\begin{tabular}{|l|}
\hline Selbstreflexion hinsichtlich Gesprächsführung \\
\hline Kommunikation als Machtinstrument \\
\hline Offenheit als Grundstrategie \\
\hline Schlechte Erfahrung mit Offenheit \\
\hline Problematische Konzepte \\
\hline Innensicht der Palliativmedizin \\
\hline Vorreiterfunktion der Palliativmedizin \\
\hline Abgrenzung zum manifesten Selbstbild der Palliativmedizin \\
\hline Ideale in der Realität \\
\hline Hemmungen beim Thema Tod \\
\hline Würde in Worte fassen \\
\hline Ethisch problematische Aspekte \\
\hline Würdevolles Sterben \\
\hline Bilder des guten Sterbens normieren Sterben \\
\hline
\end{tabular}

Anschließend wurden auch die anhand der Codes 2. Ordnung kodierten Interviewabschnitte ihrer Informationsfülle reduziert. Diese beschriebenen Arbeitsschritte dienten dazu, sich auf wesentliche Inhalte fokussieren zu können und sie für die Analyse des Materials zugänglich zu machen. Im Rahmen der Materialreduktion wurde darauf geachtet, anhand einer differenzierten Kodierung den Informationsverlust so gering wie möglich zu gestalten.

Ein Analysebeispiel soll im Folgenden zeigen, wie kodierte Textpassagen aus dem empirischen Material bearbeitet wurden. Die aufgeführten zwei Zitate wurden unter dem Code 2. Ordnung Ambivalenz von Autonomie kodiert:

Ich lasse dem Patienten schon Raum zu fragen oder sage auch mal "Haben Sie das verstanden oder haben Sie noch Fragen im Moment?" Aber Autonomie, das ist so ein Schreibtischbegriff. Patienten wollen auch nicht immer autonom sein, also echte Patienten. Es gibt natürlich für jede Menge (.) gesunder Menschen, die hierher kommen, die vor irgendwas Angst haben, die irgendwas gegoogelt haben, die wollen natürlich mitsprechen, aber wirklich 
Kranke wollen nicht autonom sein, die wollen jemanden, der sie an die Hand nimmt. (Interview 15, Abs. 35)

Also grundsätzlich ist sie [die Autonomie] eigentlich ein extrem hohes Gut, ähm, ich habe gleichzeitig (..) über die Jahre für mich persönlich festgestellt, ganz so einfach, so nach Lehrbuch, ist es nicht, ähm, also grundsätzlich ist das, was der Patient möchte überhaupt der Maßstab für alles. Also, jenseits von gesetzlichen Regelungen, die wir ja jetzt Gott sei dank ein bisschen klarer haben, ähm, ist das einfach so ein Wertemaßstab, der für mich wichtig ist. Aber ich stelle auch fest, dass Leute in Extremsituationen einfach nicht den Grad der Autonomie haben, ähm, den das Lehrbuch ihnen zugesteht. Von daher ist es immer so eine Gradwanderung zwischen, die Autonomie und die Würde des Patienten wahren und gleichzeitig aber so paternalistisch zu sein, wie es die Situation dann gerade wieder erfordert. (Interview 3, Abs. 24)

\begin{tabular}{|c|c|c|}
\hline & Paraphrase & Generalisierung \\
\hline Interview 15 & $\begin{array}{l}\text { Trotz der Intention, Autonomie zu } \\
\text { fördern, ist diese oftmals nicht } \\
\text { praxistauglich. Ernsthaft kranke } \\
\text { Patienten wollen keine } 100 \% \text {-ige } \\
\text { Autonomie hinsichtlich therapeutischer } \\
\text { Entscheidungen. }\end{array}$ & $\begin{array}{l}\text { Das Starkmachen des Autonomieprinzips } \\
\text { reicht nicht aus, dieses für die } \\
\text { medizinische Praxis handlungsrelevant } \\
\text { zu machen. }\end{array}$ \\
\hline Interview 3 & $\begin{array}{l}\text { Autonomie wird grundsätzlich ein } \\
\text { sehr hoher Stellenwert zugesprochen. } \\
\text { Schwierige Situationen verdeutlichen } \\
\text { jedoch, dass die konkrete Umsetzung } \\
\text { häufig nicht oder nur in „abgeschwächter } \\
\text { Form“ gelingt. }\end{array}$ & $\begin{array}{l}\text { Hinsichtlich theoretisch entworfener } \\
\text { Ideale, wie des Autonomieprinzips, wird } \\
\text { deutlich, dass Theorie und Praxis häufig } \\
\text { divergieren. Autonomie funktioniert nur } \\
\text { mit Hilfe unterstützender Prinzipien. }\end{array}$ \\
\hline
\end{tabular}

\begin{tabular}{|l|l|}
\hline & \multicolumn{1}{|c|}{ Materialreduktion } \\
\hline $\begin{array}{l}\text { Interview 15 } \\
\text { und 3 }\end{array}$ & $\begin{array}{l}\text { Mit Blick auf die Umsetzung des Autonomieprinzips divergieren Theorie und } \\
\text { Praxis. Autonomie funktioniert nicht als absolut gedachtes Prinzip, sondern } \\
\text { nur im Rahmen zwischenmenschlicher Beziehungen. }\end{array}$ \\
\hline
\end{tabular}


Die aus dem empirischen Material entnommenen Aussagen wurden paraphrasiert, generalisiert und schließlich reduziert. Das Paraphrasieren bestand aus der Streichung weniger oder nicht inhaltsreicher Informationen wie Wiederholungen oder Ausschmückungen und der Textübertragung in eine einheitliche Sprachebene. Die Generalisierung der Aussagen wurde genutzt, um die Paraphrasen auf ein Abstraktionsniveau zu bringen. Der Analyseschritt der Generalisierung sollte bereits einen direkten Bezug auf die zu untersuchenden Annahmen herzustellen. Im dritten Schritt wurde eine Reduktion des Materials vorgenommen, indem inhaltsgleiche Informationen zusammengefasst oder gestrichen wurden. Unter Berücksichtigung theoretischer Vorannahmen wurden nur Informationen übernommen, die von zentralem Inhalt schienen. Die entstandenen Reduktionen dienten dazu, die Auswertungskategorien aufzubauen. Das reduzierte empirische Material wurde beim erneuten Durchlesen der Interviews auf seinen Sinn überprüft. 


\section{Auswertungen}

In den Kapiteln I. und II. wurde zunächst der theoretische Hintergrund, der inhaltlich maßgebend für den Leitfaden der Qualitativen Interviewstudie war, erarbeitet. Nach dem Zwischenfazit und der anschließenden ausführlichen Darstellung des methodischen Vorgehens, sollen nun im Folgenden die medizinisch und medizinethisch relevanten Ergebnisse der vorliegenden qualitativen Interviewstudie vorgestellt werden.

Ein zu betonender Aspekt ist, dass sämtliche Ergebnisse von rein explorativem Charakter sind und dass die im Verlauf vorgestellten Hypothesen primär als Grundlage für vertiefende und an die vorliegende Arbeit anknüpfende Projekte fungieren können. Auch in Hinblick auf die theoretischen Grundlagen für das Kernanlagen der Arbeit, das Autonomieprinzip zwischen Theorie und Realität zu analysieren, soll an dieser Stelle noch eine Anmerkung formuliert werden. Neben dem Abgleich mit dem Autonomiediskurs, wie er sich in der Medizinethik vollzogen hat, wurde das Autonomieprinzip, wie es als Ideal in der Palliativmedizin theoretisch entworfen wurde, in die Analysen mit einbezogen. Im Rahmen dessen wurde herausgearbeitet, wie sich das Prinzip der Autonomie aus der Perspektive von Palliativmedizinern und kurativ tätigen Medizinern in deren Handlungswirklichkeit darstellt. Hinsichtlich der Frage nach der Realisierbarkeit des Autonomieprinzips in der medizinischen Praxis ergaben sich diesbezüglich keine wesentlichen Unterschiede, sodass in den folgenden Ergebnisteilen zu dem Prinzip der Autonomie palliativ und kurativ tätige Mediziner nicht getrennt betrachtet werden.

\section{Ergebnisse}

\subsection{Die Grenzen des Autonomieprinzips in der medizinischen Praxis}

Das folgende Kapitel widmet sich dem Verwirklichungshorizont von Autonomie in der medizinischen Praxis. Der Argumentationsfokus auf Patientenautonomie scheint als klares moralisches Leitbild zu fungieren, welches in der Lage ist, Machtstrukturen aufzulösen und dem Patienten sein Mitspracherecht in der Arzt-Patienten-Beziehung zu sichern. Nähert man sich jedoch der Praxis anhand der Schilderung beispielhaft erlebter Situationen so wird auch die Problematik eines solchen Ideals in der Konfrontation mit dem medizinischen Alltag deutlich (vgl. Woydack und Inthorn 2013). 


\subsubsection{Autonomie als anerkanntes Ideal}

Die Autonomie des Patienten, die sich in der theoretischen Debatte eindeutig gegen den ärztlichen Paternalismus richtet, wird sowohl von palliativ als auch von kurativ tätigen Medizinern als wichtigstes Element des Arzt-Patienten-Gesprächs benannt. Dies ist nicht nur für den Patienten, sondern für alle im Kontext der Krankheit betroffenen Personen, von Bedeutung. Autonomie wird als zentraler Wert anerkannt. Der Ort, an dem sich Autonomie zeigt, ist das Arzt-Patienten-Gespräch, in dem Raum für die Wünsche des Patienten ist.

\subsubsection{Spannung zwischen Theorie und Praxis im Umgang mit Autonomie}

In Hinblick auf die Schilderung konkreter Situationen am Lebensende und exemplarisch beschriebener Aufklärungsgespräche wird aus den Interviews jedoch auch deutlich, dass die befragten Ärzte zwar den Prinzipiencharakter von Autonomie grundsätzlich bejahen, gleichzeitig aber Schwierigkeiten in der Umsetzung erleben. Diese Ambivalenz zwischen Ideal und Altagserfahrung beschreibt ein Palliativmediziner wie folgt:

(Atmet tief ein, lächelt) Also grundsätzlich ist Autonomie eigentlich ein extrem hohes Gut, ähm, ich habe gleichzeitig (..) über die Jahre für mich persönlich festgestellt, ganz so einfach, so nach Lehrbuch, ist es nicht, ähm, also grundsätzlich ist das, was der Patient möchte überhaupt der Maßstab für alles. Also, jenseits von gesetzlichen Regelungen, die wir ja jetzt Gott sei dank ein bisschen klarer haben, ähm, ist das einfach so ein Wertmaßstab, der für mich wichtig ist. Aber ich stelle auch fest, dass Leute in Extremsituationen einfach nicht den Grad der Autonomie haben, ähm, den das Lehrbuch ihnen zugesteht. Von daher ist es immer so eine Gradwanderung zwischen, die Autonomie und die Würde des Patienten wahren und gleichzeitig aber so paternalistisch zu sein, wie es die Situation dann gerade wieder erfordert. (Interview 3, Abs.22, PM)

Der Palliativmediziner stellt die pauschale Zuschreibung der Fähigkeit zur autonomen Entscheidung auf Seiten der Patienten in Frage und deutet bereits an, wie unterschiedlich angemessene Reaktionsweisen von Seiten der Ärzte aussehen können.

Ähnlich kritisch äußert sich eine Gastroenterologin, die die Frage des Autonomieverlusts aufgrund der Übermittlung einer infausten Prognose aufwirft. Sie beschreibt die Problematik im Bezug auf die Rolle des Arztes im Arzt-Patienten-Gespräch: 
(..) Die Autonomie des Patienten, das ist ja sozusagen, wie gesagt, der Moment, in dem du jemandem den Boden unter den Füßen wegziehst und dann ist das eigentlich erstmal der Verlust von Autonomie. Ein absoluter Verlust. (Interview 6, Abs.21, Gastroenterologin)

Für die Ärztin ist an dieser Stelle die Reflexion in Bezug auf die Realisierung des Autonomiekonzepts von geringer Relevanz. Vor dem Hintergrund eines Autonomieverlusts wird für sie die Frage nach der Priorität von Autonomie im Rahmen eines Aufklärungsgesprächs hinfällig.

Darüber hinaus äußert sich ein hausärztlich tätiger Internist kritisch zu der Frage, in wie weit Autonomie und eine Möglichkeit zur Mitsprache überhaupt immer im Interesse des Patienten liegen. Der Arzt führt an, dass Patienten ihre Entscheidungsmacht an den Arzt abgeben und von sich aus wenig Mitspracherecht einfordern und benennt damit eine weitere Diskrepanz zwischen dem theoretischen Diskurs über Autonomie und seiner Anwendbarkeit in der Praxis. Er thematisiert, inwiefern der "Schreibtischbegriff“ Autonomie, formuliert im theoretischen Diskurs, überhaupt für die medizinische Praxis handlungsrelevant werden kann.

Ich lasse dem Patienten schon Raum zu fragen oder sage auch mal "Haben Sie das verstanden oder haben Sie noch Fragen im Moment?" Aber Autonomie, das ist so ein Schreibtischbegriff. Patienten wollen auch nicht immer autonom sein, also echte Patienten. Es gibt natürlich für jede Menge (.) gesunder Menschen, die hierher kommen, die vor irgendwas Angst haben, die irgendwas gegoogelt haben, die wollen natürlich mitsprechen, aber wirklich Kranke wollen nicht autonom sein, die wollen jemanden, der sie an die Hand nimmt. (Interview 15, Abs.35, HA)

\subsubsection{Medizinische Indikation als Grenze von Patientenautonomie}

Neben der oben geschilderten Beschränkung von Autonomie durch das jeweilige Bedürfnis des Patienten nach Fürsorge formuliert ein Onkologe eine weitere Grenzen der Patientenautonomie. Autonomie erfährt aus der Sicht des Arztes dort seine Grenzen, wo inm die ärztliche Verantwortung für Therapie und Diagnose entgegengestellt wird. Die Deutungsmacht des Arztes über das seines Erachtens nach Relevante, wie die medizinische Indikation, wird sowohl in den Schilderungen von Palliativmedizinern als auch von kurativ tätigen Kollegen als Grenze des Entscheidungsspielraums von Patienten markiert. 
Also die Autonomie ist schon $100 \%$ bei dem Patienten da. Wenn sie signalisieren "Ich will nicht darüber sprechen." oder wenn sie signalisieren "Ich will (..)" die Patientenautonomie ist bis zu dem Tod da. Allerdings gebe ich mich auch nicht in die Verfügungsgewalt eines Patienten. Also seine, die Autonomie des Betroffenen kann bleiben, aber meine eben auch. Und ich kann mich erinnern, ich hab das einmal machen lassen mit einem katastrophalen Ergebnis, dass ich mich von einem Patienten habe überreden lassen noch einen Zyklus Chemotherapie zu machen, den ich eigentlich nicht geben wollte. Das sind so sechs bis acht Zyklen, dann habe ich gesagt "Hören wir nach sechs Zyklen auf." und der Patient wollte unbedingt noch zwei weitere Zyklen haben. Er hat eine ganz schwere septische Komplikation im siebten Zyklus gehabt, den ich eigentlich schon gar nicht geben wollte. Das werde ich nie mehr tun, mir in eine Therapie hineinreden lassen. (Interview 17, Abs.31, Onkologe)

Eine Palliativmedizinerin schildert das Zusammenspiel von Patientenentscheidung und ärztlicher Indikationsstellung im informed consent als explizit moralische Aufgabe des Arztes.

Ich glaube halt, vielleicht diese, ähm, ich glaube wir müssen einen Begriff für unsere ärztliche Verantwortung bekommen. Also ähm, es gibt ja so eine Umkehr in der 70/ 80-er Jahren von dieser völligen Verantwortungsübernahme des Arztes, das Modell shared-decision-making. Das (.) klappt nicht immer gut. Es gibt eben erstmal Kulturen, äh, die sind völlig überfordert oder da ist es kulturell nicht etabliert, dass man shared-decision-making mit einer jungen Frau macht. Ich stelle mir jetzt so eine türkische Großfamilie vor, wo wir jetzt sagen "Wir gucken jetzt mal zusammen oder wir besprechen genau was der richtige Weg ist." Da entsteht eine große Unsicherheit bei der Familie und die denken irgendwie so die kann das nicht entscheiden, ja? Ich glaube, ähm, ich muss, also gerade diese Entscheidung, ist eine Intensivstation indiziert oder nicht? Ist eine Chemotherapie noch indiziert oder nicht? Die muss ich erstmal als Arzt für mich alleine treffen. Und wir neigen, finde ich eher im Moment, dazu zu sagen, gerade bei der Therapieeskalation, so bei der 6.-LinienChemotherapie "Der Patient wollte aber unbedingt." und, ähm, da denke ich müssen wir Ärzte klarer werden in unseren Aussagen und uns auch trauen zu sagen "Eine Chemotherapie ist jetzt nicht mehr indiziert." Punkt, ja? So. Und 
ähm, natürlich weiß ich immer, also es gibt Situationen, wo ich denke da muss ich einfach Verantwortung übernehmen. (Interview 16, Abs.35, PM_in)

\subsubsection{Imperativ der Eigenverantwortung des Arztes}

Neben den verschiedenen Beschränkungen von Patientenautonomie und den generellen Schwierigkeiten mit dem Autonomieprinzip im medizinischen Praxisgeschehen, nennen die befragten Ärzte das klare Ziel, Patientenautonomie am Lebensende fördern und erhalten zu wollen. Innerhalb der Spannung zwischen Autonomie als Ideal, das sowohl palliativ als auch kurativ tätige Mediziner bejahen, und den kritischen Äußerungen hinsichtlich der Umsetzung des Autonomieprinzips im komplexen Praxisgeschehen, positionieren sich die Ärzte mit Blick auf ihre Intention relativ eindeutig. Sie beschreiben es als ihre Aufgabe, Autonomie mehr Bedeutung im medizinischen Alltag zu geben. Gerade vor dem Hintergrund der thematisierten Schwierigkeiten scheint es zudem Konsens zu sein, dass es hierzu mehr bedarf als dem Patienten seine individuelle Selbstbestimmung wohlwollend zurückgeben zu wollen. In dieser Hinsicht formuliert ein Internist eine Art Imperativ der ärztlichen Eigenverantwortung in der Gestaltung der Entscheidungssituation.

Also ich glaube meine Aufgabe als Arzt ist es, den Patienten so gut zu informieren, dass er vielleicht auch selber die Entscheidung treffen kann, shared decision making nennt man das ja, also dahin zu kommen. Trotzdem bin ich als Arzt auch gefordert ihm eine Empfehlung zu geben." (Interview 9, Abs.23, Onkologie)

Die Entscheidung des Patienten in sinnvoller Weise zu unterstützen - hier durch eine Empfehlung - kann eine Möglichkeit sein, seine Autonomie zu wahren und zu unterstützten. Einen Schritt weiter geht ein Palliativmediziner, der die Facetten des Prinzips der Autonomie anspricht und dabei darauf eingeht, dass die Patientenautonomie insbesondere bei Problemen und Fragen am Lebensende häufig eine ganz eigene Form annimmt. Er beschreibt in seiner Überlegung die Bedeutung von Rahmenbedingungen für Autonomie und kritisiert theoretische Vorstellungen von Autonomie als Unabhängigkeit.

Also Patientenautonomie am Lebensende das ist ja das Thema in der Palliativmedizin schlechthin, ja? Und es ist ja so, man versucht ja, äh, das hat ja Cicely Saunders schon so formuliert "to maintain the own character in style until the end." Gerade das ist das Wichtige, dass jeder eine Autonomie behält, denken wir. Das Problem ist, dass viele Patienten gar nicht mehr in der Lage 
sind, autonome Entscheidungen zu treffen oder sich eben Hilfe wünschen in der Situation, dass durch die Hilfe oder Unterstützung eine Autonomie wieder möglich ist. [...] Und Autonomie heißt ja eigentlich ungestützt, selbstständig leben - so empfinden wir das, ja? Aber die Autonomie am Lebensende ist häufig eine ganz andere Autonomie, also Entscheidungen aufgrund einer Unterstützung oder auch Hilfestellung bei der Entscheidungsfindung. (Interview 19, Abs.27, PM)

Im Sinne eines Imperativs der Eigenverantwortung ist es - neben der Betonung der Patientenautonomie - auch für die ärztliche Seite erforderlich mit einer zielführenden Vorstellung in ein Gespräch zu gehen und stets erst den Anspruch an sich selbst zu erheben, sein gegenüber mit seinen Vorstellungen von Partizipation und Selbstbestimmung vollständig zu verstehen. Darüber hinaus sollte der Aspekt der Berücksichtigung und Wahrung von Patientenautonomie niemals gleichbedeutend damit sein, als Arzt nicht mehr in einer gewissen Verantwortungspflicht für seine Patienten zu sein.

Das empirische Material zeigt insgesamt das Bestreben auf Seiten der Ärzte, Autonomie als Prinzip anzuerkennen und die Selbstbestimmung des Patienten im komplexen Medizinkontext zu erhalten, beziehungsweise dem Patienten wieder zu geben. Gleichzeitig wird von den Interviewpartnern deutlich auf die Grenzen einer solchen als Ideal formulierten Vorstellung von Autonomie hingewiesen. Die Selbstbestimmung des Patienten wird dabei im konkreten Handlungsspielraum des Arztes, insbesondere im ArztPatienten-Gespräch, betrachtet, vor dessen Hintergrund auch die Grenzen der Selbstbestimmung thematisiert werden.

Vor dem Hintergrund der beiden skizzierten Diskurslinien zu Autonomie - in theoretischen Positionen zur Palliativmedizin und in dem Diskurs der Medizinethik - verdeutlichen die empirischen Ergebnisse, dass in der Praxis vor allem auf das Ideal der Autonomie, wie es im Diskurs der Palliativmedizin angenommen wird, Bezug genommen wird. Dieses Ideal wird in den Interviews problematisiert, da in der Praxis die Autonomie des Patienten als durch verschiedene Faktoren begrenzt erlebt wird. Theoretische Positionen der Kritik an einem solchen Ideal der Autonomie, wie sie beispielsweise im Ansatz der relationalen Autonomie formuliert wird, spielen dabei in den Aussagen der Interviewpartner keine Rolle. Umgekehrt zielen die beiden theoretischen Diskurse darauf ab, Autonomie im Sinne eines universalen Prinzips zu etablieren und zu problematisieren. 
Eine Verzahnung der drei Diskurse - dem Diskurs zur fachlichen Ausrichtung der Palliativmedizin, medizinethische Überlegungen und die Reflexion der medizinischen Praxis - scheint weitgehend zu fehlen. Die geschilderten Spannungen, wie sie sich zwischen palliativmedizinischer Grundlagenliteratur und der medizinethischen Debatte zu dem Prinzip der Autonomie finden, belegen die Notwendigkeit der theoretischen Auseinandersetzung mit dem Prinzip der Autonomie. Gleichzeitig zeigen die empirischen Daten, dass die Kritik am Autonomieprinzip in der Praxis nicht rezipiert, aber anhand von Erfahrungen in ähnlicher Weise zum Thema gemacht wird. Dabei signalisieren die praktizierenden Ärzte sowohl Diskussionsbedarf als auch die Bereitschaft zur Auseinandersetzung und Weiterentwicklung der Patientenautonomie.

Die Interviews verdeutlichen das Bestreben, einen differenzierten Umgang mit Autonomie im medizinisch-praktischen Alltag von ärztlicher Seite zu unterstützen. Die Ärzte erheben aber weder den Anspruch, jedes Detail der medizinethischen Debatte mitführen zu können, noch sollen die skizzierten Grenzen der Autonomie dazu führen, Formen des Paternalismus zu rechtfertigen. Durch eine verstärkte Verschränkung der drei Diskurse könnte eine wechselseitige Bereicherung und Erweiterung erfolgen, die das Bestreben der Ärzte in dieser Richtung unterstützt und sich auch positiv auf die Praxis auswirkt. Der medizinethische Diskurs würde sensibilisiert, die eigene Debatte stärker auf die konkreten Probleme der Umsetzung und graduellen Einschätzung konkreter Situationen zu fokussieren und die oft vorherrschende Distanz zwischen den Betroffenen und denen, die die Debatte führen, zu reduzieren. Hinsichtlich des medizinisch-praktischen Alltags kann das Ideal der Autonomie als Korrektiv dienen, um sowohl kurativ als auch palliativ tätige Ärzte dahingehend zu sensibilisieren, mehr Raum für den Subtext der (Arzt-)PatientenKommunikation zu ermöglichen und jenseits des informed consent nach Patientenautonomie zu fragen und somit Autonomie im Sinne gestützter Selbstbestimmung zu verstehen (vgl. Woydack und Inthorn 2013).

Bei einer aus dem Kontext heraus verstandenen Selbstbestimmung werden Rahmenbedingungen thematisierbar, die sowohl von Seiten der Praxis als auch von Seiten der Ethik reflektierbar sind. Die Überlegungen können helfen, Situationen einzuschätzen und nach konkreten Möglichkeiten und Mitteln der Gestaltung zu fragen, die Autonomie des Patienten im Arzt-Patienten-Gespräch zu stärken. Bei Beachtung und Reflexion dieses Modells wird die Autonomie des Patienten nicht geschwächt, sondern gerade gefördert und unterstützt (ebd.). 


\subsection{Autonomie in Wechselwirkung zu professionsspezifischen Handlungslogiken}

In dem nachfolgenden Kapitel werden die wesentlichen Hypothesen vorgestellt, die nicht primär theoriegeleitet, sondern induktiv aus den Analysen des empirischen Materials entstanden sind. Hervorzuheben sind im Rahmen dessen die internen Handlungslogiken, die die einzelnen Fachdisziplinen in ihrer Kommunikation und in ihrem Umgang tiefgehend prägen. Bevor auf diesen Aspekt näher eingegangen wird, soll jedoch ein Blick auf die im Rahmen der Interviews zur Sprache gekommenen Aspekte zur Arzt-Patienten-Interaktion und zu Aspekten der Kommunikation erfolgen.

\subsubsection{Verflechtung von Autonomie und Kommunikation als Instrument}

Im Rahmen der vorliegenden Arbeit lag während des gesamten Forschungsprozesses ein Hauptaugenmerk auf den Kommunikationsstrategien von praktizierenden Medizinern. Mit Blick auf die Realisierbarkeit des Autonomieprinzips wurden diese selbst, sowie auch die Selbstreflexion über die eigenen Kommunikationsstrategien dabei im Sinne eines Werkzeugs der Praxis betrachtet.

Um darzustellen, welche Rolle Kommunikation für praktizierende Mediziner spielt, wird zunächst das empirische Idealbild des Arzt-Patienten-Gesprächs mit Fokus auf die Aufklärung über eine infauste Prognose betrachtet. In einem nächsten Schritt sollen die generell als wesentlich empfunden Strategien und Ziele in der Arzt-PatientenKommunikation dargestellt werden. Abschließend werden Schwierigkeiten und Konfliktsituationen diskutiert, die mit der Etablierung einer Idealvorstellungen für das ArztPatienten-Gespräch bei Fragen am Lebensende einhergehen.

\subsubsection{Das Idealbild des Arzt-Patienten-Gesprächs}

Eine häufig zitierte Idealvorstellung als Einstieg in ein schwierigeres Aufklärungsgespräch ist, sich als Arzt zunächst ein Bild davon zu machen, auf welchem Wissensstand der Patient sich befindet, um im Verlauf auf diesem Wissen aufbauen zu können. Dabei scheint Offenheit gegenüber den Gefühlen und Bedürfnissen des Patienten ein wichtiger Aspekt zu sein. Neben dem Aspekt der Offenheit wird jedoch vielfach erwähnt, dass ein zurückhaltender Umgang mit Vokabeln wie „Krebs“ oder „Sterben“ für den Verlauf eines Arzt-Patienten-Gesprächs von Vorteil zu sein scheint.

Vielfach wird beschrieben, dass es initial vor einem Aufklärungsgespräch über eine infauste Prognose wertvoll ist, seinen Patienten zunächst kennen zu lernen. Exemplarisch beschriebt eine Palliativmedizinerin folgendes: 
Ich versuche schon, ähm, eben halt zu schauen, dass ich mich erstmal orientiere, was will der Patient hören, also, wo, wie kommt der rein? Wie kommt der in ein Gespräch rein? Ähm, mit welche Gefühlen, mit welchen Informationen er heran tritt, wie offen er ist, wie detailgenau er eine Aufklärung braucht (..) ich versuche in einem ersten Kontakt erstmal den Patienten kennen zu lernen und, äh, dann geht es meistens nicht um Aufklärung und dann versuche ich ihn auch darauf vorzubereiten, wenn es um Aufklärung geht eben über eine Verschlechterung oder eine Begrenzung oder eben halt das Sterben. (Interview 16, Abs.7, PM_in)

Neben dem Fokus auf Offenheit wird, wie in dem folgenden Zitat aufgeführt, mehrfach betont, dass es von Bedeutung ist, sich ein Bild davon zu machen, wie viel man seinem Gegenüber im Rahmen des Gesprächs zumuten kann. Häufig thematisiert wird zudem das notwendige Bewusstsein über den potentiellen Gesprächsverlauf abhängig von der Persönlichkeit seines Gegenübers. Neben dem Bewusstsein über eine sowohl fachliche als auch organisatorische Vorbereitung auf ein Gespräch über eine infauste Prognose ist also das theoretische Bewusstsein für die wesentliche zwischenmenschliche Ebene auf Seiten der Mediziner vorhanden.

Wie viel trägt der, also ich sollte die Leute kennen, und wie viel kann ich ihnen zumuten, wie viel wollen die meiner Einschätzung nach wissen, einen Raum dafür zu haben, Zeit dafür zu haben und vor allen Dingen mit den medizinischen facts Sicherheit haben. (Interview 17, Abs.7, Onkologie)

\subsubsection{Subtext des Patienten als Variable}

Mit der Intention, den Gesprächsverlauf möglichst nahe an den Bedürfnissen und dem Wissensstand des Patienten entlang zu gestalten, wird vielfach der Versuch geschildert, auf Dinge einzugehen, die zunächst nur subtil von Seiten des Patienten ins Gespräch gebracht werden. Der wahrgenommene Subtext des Patienten im Verlauf eines Gesprächs führt in den meisten Fällen zu schwierigen Themen wie dem Sterben und der Angst vor dem, was die jeweilige Diagnose im Einzelfall mit sich bringt. Ein häufig erwähnter Aspekt ist der seitens des Patienten nur subtil angesprochene Wunsch, über die Prognose der Erkrankung zu sprechen. Wie hoch die Deutungsmacht über derartige Aspekte auf Seiten des Arztes ist, beschreibt ein Palliativmediziner wie folgt: 
[...] Ja, das wird, glaube ich, von Patienten, äh, gerne angefragt. Aber nicht so konkret. Die Frage eines Patienten ist eher "Und wie geht es jetzt mit mir weiter, Herr Doktor?" Und das können Sie als Arzt oder Ärztin natürlich sehr schnell sagen "Ja, wir haben ja jetzt beschlossen, morgen wird noch einmal das Röntgenbild gemacht und dann wollen wir noch das Antibiotikum austesten und dann, äh, gucken wir, wie die Verlegung ist oder ich kann sagen "Sie wissen, dass die Erkrankung nicht heilbar ist, wir sehen auch, dass es einen deutlichen Progress, einen Fortschritt dieser Erkrankung gibt, Sie werden daran sterben." Eine Frage, zwei komplett unterschiedliche Wege, wie Sie darauf reagieren und wie Sie in der Lage sind, etwas hören zu wollen, hören zu können, und das entscheidet die Kommunikation mit dem Patienten. (Interview 21, Abs.31, PM)

Anhand des Beispiels ist eindrücklich zu sehen, inwiefern mit einer ausweichenden, möglicherweise $\mathrm{zu}$ wenig überlegten Antwort seitens des Arztes ein elementarer Themenbereich möglicherweise komplett ausgeblendet wird. Mit der ärztlichen Entscheidung, ob über kleine Behandlungsdetails oder über die schlechte Prognose der vorliegenden Erkrankung gesprochen wird, unterscheidet sich die Ebene der Kommunikation maßgeblich.

\subsubsection{Offenheit als Grundstrategie}

Im Umgang mit schwierigen Themenbereichen wie den Fragen am Lebensende und der Aufklärung darüber, dass bestimmte Diagnosenstellungen unweigerlich den Tod zur Folge haben, wird im Zuge einer Ethisierung der Medizin von praktizierenden Medizinern die Fähigkeit verlangt, offen über die genannten Themen kommunizieren zu können und keine Hemmungen im Umgang mit dem Tod zu haben. Die Zielsetzung, Offenheit in ein Gespräch zu etablieren, wird vielfach rezipiert und oftmals in engem Zusammenhang mit der Förderung von Patientenselbstbestimmung gesehen. In diesem Zusammenhang wird vielfach eine Art Anspruch des Patienten auf Informationen betont, damit diese Klarheit für das eigene Bewusstsein erlangen und die Chance haben, sich auf den eigenen Tod einstellen zu können.

Ein Onkologe konkretisiert beispielhaft seine Deutlichkeit gegenüber Patienten. Darüber hinaus kommt an dieser Stelle der Aspekt zur Sprache, dass es im Rahmen eines Offenheitskonzepts - neben der Aufklärung über die Diagnose - von großer Bedeutung ist, 
eine ehrliche Einschätzung darüber zu bekommen, was im Rahmen der Erkrankung medizinisch möglich ist und was nicht.

"So und so geht es und so weit kommen wir noch und so weit nicht mehr." und dass ich eben nicht immer wieder sage, jetzt zaubere ich noch einmal etwas aus dem Ärmel und jetzt können wir noch was tun (.) und nie zu sagen "Es geht einfach nicht mehr, Sie werden daran sterben." (Interview 9, Abs.71, Onkologe)

\subsubsection{Schlechte Erfahrung mit Offenheit}

Im Verlauf der Untersuchungen wurde jedoch deutlich, dass sich bei der Umsetzung dieses Ideals zum Teil auch Probleme in der Arzt-Patienten-Interaktion ergeben können. In der medizinischen Praxis zeigt sich, dass Ärzte, die dem Imperativ einer Ethisierung der Medizin folgen, schlechte Erfahrungen machen, wenn sie offen die Grenzen des medizinisch Machbaren thematisieren oder sehr offensiv das Thema Sterben und Tod angehen. Die Resonanz der Patienten zeigt, dass hier scheinbar Handlungsweisen etabliert werden, die dem Patienten tendenziell das Gefühl geben einen Arzt vor sich zu haben, der nicht mehr bereit ist, alles medizinische Mögliche zu tun. Ein Aspekt, der im konkreten Praxisgeschehen negativ bewertet wird.

So schildert eine Palliativmedizinerin bezüglich ihrer Erfahrung mit Offenheit folgendes:

Dann gab es eine Situation, wo wir das Gefühl hatten, jetzt hat sich der Zustand erheblich verschlechtert, ähm, der Patient könnte in den nächsten Stunden, Tagen sterben. Und die Schwester hatte schon die Frau informiert und wir kamen auch gleich dahin und standen dann plötzlich vor Frau und Patient, die sich so angucken "Was ist denn jetzt hier los? Wir wissen gar nicht." und ähm, da habe ich extrem klare Worte gewählt, also ich habe gesagt "Wir haben Ihre Frau benachrichtigt, weil wir uns Sorgen machen um Ihr Leben, wir sehen wie schwach Sie jetzt sind, das ist hat sich extrem verändert in der letzten Woche und wir haben Angst, dass Sie bald sterben könnten. Und wir wollen, dass auch Ihre Familie informiert ist." Da war der Patient richtig wütend. (Interview 16, Abs.19, PM_in)

Eine deutlich negativ erlebte Resonanz seitens des Patienten auf einen offenen Umgang mit dem Sterben beschreiben auch eine Gastroenterologin und ein Palliativmediziner. 
Und dem habe ich gesagt, dass der sich das überlegen muss, wie er das weiter gestalten will. Der war einfach völlig schockiert und hat nur in Abwehr reagiert. (Interview 6, Abs.99, Gastroenterologin)

Und wir machen dann halt das Aufnahmegespräch und fragen natürlich dann auch so ein bisschen standardmäßig unsere Aufnahmebögen ab und da geht es dann auch zum Beispiel um Patientenverfügung und Vorsorgevollmacht. Das äh, und diese Frage hat diesen Patienten total aus der Bahn geworfen, hatte ich das Gefühl, also dass ich nach diesem Thema fragte, ob er das hat (.) war er ganz erstaunt und sagte "Muss ich das denn schon haben?" und ähm, "Ich dachte eigentlich, dass ich noch zehn Jahre lebe." (Interview 13, Abs. 43, PM)

Ein weiter Palliativmediziner, dem Offenheit und Aufrichtigkeit gegenüber Patienten mit infauster Prognose generell wichtig ist, betont explizit, dass ein offenes Gespräch über Themen wie Sterben und Tod nur gangbar ist, wenn man es sanft etabliert und den Patienten nicht nach der eigenen Vorstellungen oder aufgrund eines vorgefertigten Gesprächskonzertes überfordert.

Aber Wahrnehmung über die Jahre ist auch, dass einfach explizit direkt ungefragt über Sterben reden häufig irgendwelche Rolladen runter gehen lässt, die ich gerne noch eine Zeit lang offen hätte. Um einfach ein bisschen flexibler in der Themenwahl zu sein, wobei viele von sich, also da gibt es durchaus Patienten, die von sich aus sagen "Ich weiß, dass ich nicht mehr lange habe." und einfach möglichst, von der Thematik her offen zu sein für das, was der Patient dann im Gespräch überhaupt für wichtig erachtet." (Interview 3, Abs. 12, PM)

Der in dem zuletzt aufgeführten Zitat betitelte Tod als „Schockfaktor“ wird in diesem Zusammenhang zwar eher plakativ beschrieben, betont jedoch einen Aspekt, der auch in den vorangegangenen Zitaten sichtbar wird, sehr deutlich. Nämlich die offensichtlich eintretenden Negativerfahrungen, die die Akteure des Medizinsystems mit dem Versuch, eine Art Offenheitskonzept zu etablieren, machen. Darüber steht eine Idealvorstellung, die innerhalb des bestehenden Medizinsystems, den Fokus auf die Autonomie des Patienten stärken soll.

Wie einleitend erwähnt, fragt das vorliegende Forschungsanliegen explizit nach der Realisierbarkeit eines derartigen Ideals und stößt dabei auf deutliche Spannungen 
innerhalb des Praxisgeschehens. Dass sich bei der Etablierung eines Ideals, welches im weiteren Sinne die Funktion eines Korrektivs mit sich bringen soll, Spannungen ergeben, ist wenig irritierend. Es scheint an dieser Stelle jedoch besonders wichtig zu sein, die Überlegung nicht aus dem Blick zu verlieren, was man anhand der Spannungen zwischen den Erwartungen an ein Korrektiv und der Wirklichkeit lernen kann, um einen Nutzen für die Akteure des Praxisgeschehens gewinnen zu können.

Neben dem Bewusstsein darüber, dass der Entstehung des Fachbereichs Palliativmedizin mit seinen Idealen für den Umgang mit Situationen am Lebensende eine vielschichtige gesellschaftliche Dynamik vorangegangen ist, scheint an diesem Punkt ein weiterer Gedankengang interessant zu sein. Die Frage nach einem der initialen Auslöser für die Entstehung einer neuen Instanz im bestehenden Medizinsystem erleichtert es, entstehende Schwierigkeiten und Spannungen klarer aufschlüsseln zu können, um mit Hilfe dieser greifbarere Lösungsansätze zu formulieren. Betrachtet man nun die Dynamik im bestehenden Medizinsystem, die mit der Entwicklung des neuen Fachbereichs einhergegangen ist, ließe sich die Hypothese formulieren, diese sei durch die interne Kritik der Bioethik am Medizinsystem entstanden. Bei dem Versuch, der Erwartungshaltung gerecht zu werden, die Förderung und das Realisieren von Autonomie in der Praxis umzusetzen, kommt es nicht nur zu Spannungen, sondern zum Teil sogar zu einem Bruch zwischen Idealvorstellungen und ärztlichen Handlungslogiken. So wird unter Berücksichtigung der Hypothese, dass Kommunikation an sich bereits Autonomie fördernd ist, in genau dieser Zielsetzung bereits eine Dilemma deutlich: Laut Maio, der an dieser Stelle exemplarisch als einer der Kritiker aufgeführt wird, besteht das Problem, dass Ärzte im Zuge der zunehmend technologisierten Medizin den Tod nicht mehr akzeptieren (vgl. Maio 2013). Im theoretischen Idealbild wie dem der Palliativmedizin ist der Arzt genau dazu jedoch fähig und ermöglicht den offenen Diskurs hinsichtlich des Themas Sterben und Tod. In der medizinischen Praxis zeigt sich allerdings, dass Ärzte, die diesem Imperativ folgen, schlechte Erfahrungen machen, wenn sie offen die Grenzen des medizinisch Machbaren thematisieren oder das Thema Sterben und Tod zu offensiv angehen. Die Gesprächspartner stoßen, wie anhand des empirischen Materials deutlich wird, beide an ihre Grenzen und registrieren unter anderem durch eine negative Resonanz, besonders auf Seiten des Patienten, dass ein Weiterarbeiten gemäß der eigenen, bisher bestehenden Handlungslogik erst durch die Akzeptanz von Verdrängungsmechanismen möglich ist und eben nicht über eine bedingungslos offene Kommunikation. Auf genau diese Paradoxie hat die Medizin möglicherweise mit der Ausdifferenzierung eines neuen Fachbereichs reagiert, nämlich mit dem Fachbereich 
Palliativmedizin. In Hinblick auf die Kritik und die Erwartungen der Öffentlichkeit, es müsse ein besserer Umgang mit dem Sterben realisiert werden, ließe sich demnach die Hypothese formulieren, die Palliativmedizin als eigenständiger Fachbereich sei somit als Reaktion auf die Kritik der Bioethik und die Erwartungen der Öffentlichkeit an das Medizinsystem entstanden. Und mit ihr der klare Fokus auf die Förderung von Patientenselbstbestimmung am Lebensende, was an dieser Stelle exemplarisch als ein Beispiel für die Säulen der Palliativmedizin steht. Wichtig zu erwähnen ist jedoch, dass dies nur ein Teilaspekt einer Gesamtdynamik sein kann. Mit der Dynamik, die zur Entstehung eines neuen Fachbereichs beigetragen hat, wurde eine generelle gesellschaftliche Forderung verfolgt und aufgenommen, die tendenziell für eine eher universal gültige Vorstellung des Autonomieprinzips steht und in diesem Zuge die Tendenz mit sich bringt, Schablonen dafür entstehen zu lassen, wie beispielsweise ein guter Umgang mit Sterbenden auszusehen hat.

\subsubsection{Problematische Konzepte}

Die im vorangegangenen Kapitel dargestellten Überlegungen werden anhand der folgenden Zitate zusätzlich untermauert. Es wird im Rahmen der erzählten Wahrnehmung eine Überforderung der Akteure des Medizinsystems erkennbar, die aufgrund der Erwartungshaltung, jeder müsse mit dem Ziel, Patientenautonomie zu fördern, offen über Sterben und Tod kommunizieren können, ausgelöst wird. So schildert eine Gastroenterologin ihre fragwürdige Strategie, offen über eine infauste Prognose aufzuklären, nachdem sie nach wenigen vergangenen Behandlungstagen selbst überrascht war, wie schnell sich der Zustand ihrer Patientin verschlechtert habe.

Dann denkt man, na Gott sei dank habe ich ihr schon einmal gesagt, dass (.) das immer progredient ist. Sie weiß 50 Lymphknoten von 60 befallen oder so, äh, die sind meist auch medizinisch gebildet natürlich. (Interview 7, Abs.17, Gastroenterologin)

Eine andere Internistin beschreibt ebenfalls ein nicht funktionierendes Konzept, welches eigentlich aus dem wertvollen Anspruch heraus offensichtlich wird, dass es ihr wichtig ist, dass ihre Patienten alles verstehen.

I: Sie sagten gerade, es ist Ihnen wichtig, dass die Patienten alles verstehen. Wie finden Sie heraus, ob wirklich Klarheit herrscht? 
B: (...) Ja, schwierig. (..) Also, eigentlich merke ich es daran, wenn die das selbst noch einmal wiederholen, aber ich, äh, ich fordere die nicht auf, das zu wiederholen. Das ist eigentlich, ja, merkt man teilweise erst hinterher, was falsch verstanden wurde. (Interview 6, Abs. 19, Gastroenterologin)

Auch ein hausärztlich tätiger Mediziner betont die Wichtigkeit, offen und aufrichtig mit den Patienten umzugehen. Bei dem Vertiefen des Gesprächs hinsichtlich konkreter Strategien wird jedoch klar, dass seine Art der offenen Kommunikation im Praxisgeschehen deutlich verhaltener ist als er es zunächst schilderte und eher in Form einer Reaktion auf die Fragen seiner Patienten stattfindet als im Sinne einer selbst intendierten Offenheit.

Joah. Es sind natürlich bestimmte Wörter, die man vermeidet. Man sagt eher Tumor als Krebs, erstmal (.) Wie gesagt hier in so einer Praxissituation hat man ja allenfalls mal im Ultraschall einen hochgradigen Verdacht und da sagt man dann "Ein Tumor, der kann auch bösartig sein. Wir wissen es aber nicht, wir müssen weiter Bildgebung machen." In der Zeit hat der Patient dann ja auch Gelegenheit, sich schon einmal damit anzufreunden, mit dem Gedanken, um es so zu nennen, oder sich damit zu befassen. Und ähm, meistens fragen die dann auch relativ konkret "Kann es auch bösartig sein?" dann sage ich "Ja." (..) Ja, die Zeiten, in denen man einem Patienten irgend etwas verheimlicht hat sind ja lange vorbei. (Interview 15, Abs.11, HA)

In dem letzten Zitat wird der zunächst als inhaltsleere Positivwert assoziierte Autonomiebegriff wie selbstverständlich an das negativ assoziierte Problem der mangelnden Compliance gekoppelt. Auch wenn dieser Aspekt sich in der Studienergebnissen nur vereinzelnd wiederfindet, könnte er darauf hinweisen, dass innerhalb der Medizin autonome Handlungslogiken vielfach dem widersprechen, was man als gesamtgesellschaftliche Werte kennt und akzeptiert. Anhand des empirischen Materials wird dieser Aspekt wie folgt sichtbar:

I: Und wenn wir noch einmal darauf blicken was für Sie Autonomie fördernde Elemente sind, gibt es da noch mehr Aspekte neben der "sachlichen klaren Aufklärung" und dem "sich Zeit nehmen" für Sie?

B: Ja, ja. Ja ja. Ich sage denen schon "Also hören Sie zu, wenn Sie diese Dosis nicht vertragen, sagen Sie bescheid. Dann reduzieren wir die Dosis. "Das ist ja schon, der Patienten sagt, verstehen Sie? Er gibt bescheid, manchmal setzt 
ein Patient auch einfach mal irgendwas ab, die Tabletten, und so, das finde ich sehr autonom. (Interview 7, Abs.35, Gastroenterologin)

Die beschriebene Interaktion kann sicher nicht ausschließlich als Akt des medizinischen Paternalismus gedeutet werden, sondern lässt auch danach fragen, warum in diesem Bereich offenbar Dinge, die in anderen gesellschaftlichen Bereichen eindeutig positiv assoziiert werden, hier problematisch sein können.

Abschließend lässt sich feststellen, dass zum Teil Konzepte geschildert werden, die von außen betrachtet nicht plausibel sind und nicht funktionieren. Durch die Zielsetzung, Offenheitskonzepte zu etablieren, ist für beide Seiten, sowohl für die Patienten als auch für die Ärzte, eine Überforderung ersichtlich.

\subsubsection{Einschränkung von Autonomie durch Hemmungen vor dem Tod}

Bei der Betrachtung der dargestellten Konflikte fällt ins Auge, dass diese sich bei dem Thema Tod sowie bei der Kommunikation über die Grenzen des medizinisch Machbaren besonders zuspitzen.

Letztlich geht es im Rahmen einer schrittweise entstehenden Ethisierung der Medizin sowie im Rahmen der Entstehung des Fachbereichs Palliativmedizin um einen guten Umgang mit dem Tod. Im Verlauf einer derartigen Dynamik werden stets Schablonen und Idealvorstellungen entwickelt, die zwar nicht den Anspruch erheben, von jedem erfüllt zu werden, sich unterbewusst aber dennoch zu einer Art Maßstab entwickeln.

Wie bereits geschildert wird jedoch eine Überforderung der Akteure des Medizinsystems erkennbar. Ausgelöst wird diese primär aufgrund der Erwartungshaltung, jeder müsse mit dem Ziel, Patientenautonomie zu fördern, offen über Sterben und Tod kommunizieren können. Dabei wird im Rahmen der Reflexion immer wieder deutlich, dass grundlegende Ängste den Umgang und die Art der Kommunikation prägen.

Feststellung ist, zum einen, diesen letzten Schritt in der kognitiven Wahrnehmung viele Patienten schon weitestgehend lange heraus schieben und viele Kollegen ziemlich massive Ängste haben, so aus der eigenen Hilflosigkeit heraus, das mit den Patienten anzusprechen. (Interview 3, Abs.3, $P M)$

So thematisiert ein Palliativmediziner nicht nur die bestehenden Hemmungen im Rahmen eines laufenden Gesprächs, sondern betont, dass derartige Arzt-Patienten-Gespräche häufig gar nicht erst zustande kommen. Wie er weiter ausführt geht es dabei nicht nur um 
eine spontane Angst vor dem Gesprächsverlauf, sondern seines Erachtens nach auch um die Selbstwahrnehmung und das persönliche Rollenbild des Arztes, das ursprünglich, dem ärztlichen Ethos folgend, zum Ziel hatte, gegen den Tod und für das Leben zu kämpfen. Ein anderer Palliativmediziner geht in seiner Überlegung näher auf die Konsequenz von potentiellen Ängsten ein. Wie häufig erwähnt geht es dabei um den Umgang mit Emotionen, der in der schulmedizinischen Ausbildung nur sehr wenig Raum findet. Dies hat zur Folge, dass praktizierende Mediziner Gespräche über das Lebensende lieber abdelegieren - ein Aspekt, auf den im Verlauf noch im Detail eingegangen wird.

Naja, es gibt verschiedene Gründe dafür wahrscheinlich. Bei manchen Ärzten immer noch einfach die Sorge, dass man dem Patienten, wenn man so etwas schlimmes anspricht, erst recht Schaden zufügt, dann ist natürlich die Sorge da, dass derjenige, der die schlechte Nachricht überbringt natürlich auch der Buhmann ist und dann gibt es natürlich viele Ängste von Seiten der Ärzte. "Wenn ich das jetzt so anspreche, ähm, dann bricht der in Tränen aus, wie gehe ich dann mit den Emotionen um?" viele Ärzte tun sich da sehr schwer und "Ich habe doch überhaupt nicht die Zeit, wenn ich jetzt auf der Visite nur ein paar Minuten habe, dann damit umzugehen, das soll lieber die Psychologin machen." Teilweise sicher auch eigene Berührungsängste mit dem Thema Sterben und Tod, ähm, weil das natürlich auch immer einen selber betrifft. (Interview 20, Abs.63, PM)

Den erwähnten Hoffnungsaspekt thematisiert auch eine Palliativmedizinerin. Während es für sie oberste Priorität hat, dem Patienten gegenüber aufrichtig zu sein, was seine gegebenenfalls schlechte Prognose anbelangt, geschieht es ihrer Erfahrung nach regelmäßig, dass ärztliche Kollegen aus einer persönlichen Überzeugung heraus eigenmächtig entscheiden, in welchem Umfang sie dem Patienten Informationen zu kommen lassen.

Ja, also, ich ähm, also es gibt hier auch in der Klinik, gerade im chirurgischen Bereich gibt es sicherlich auch Chefärzte, die fest davon überzeugt sind, dass wir nicht über Prognose reden dürfen und dass wir den Patienten damit die Hoffnung nehmen und dass, ähm, dass der Patient sich dann nicht mehr auf das Leben einstellen kann, dass er, wenn er jetzt diese Operation überstehen muss aber erstmal hoffen muss. Und dass finde ich ganz (.) das finde ich, 
ähm, das finde ich einen einen Kunstfehler, ja? So weil ich denke das ist kein Respekt vor dem Gegenüber. (Interview 16, Abs.59, PM_in)

Während beispielsweise bei geplanten medizinischen Eingriffen absolut verbindlich nur nach dem Prinzip des informed consent gehandelt werden darf, liegt die Deutungsmacht hinsichtlich der Grenzen zwischen kommunizierbaren und nicht-kommunizierbaren Themen eindeutig auf der Seite des Arztes.

\subsubsection{Normierungsgefahr durch Schablonen für das Sterben}

In der Diskussion um einen sogenannten würdevollen Tod, der im Zuge einer Ethisierung der Medizin vielfach Thema ist, tritt aus den Schilderungen des empirischen Materials immer wieder hervor, dass dieser etwas sehr individuelles und wenig beschreibbares ist. Zudem betonen die praktizierenden Mediziner, wie es exemplarisch in dem folgenden Zitat zu sehen ist, dass momentan weder ein Konzept noch ein besonders spezialisierter Fachbereich in der Lage zu sein scheint, einen guten oder würdevollen Tod garantieren zu können. Ein Palliativmediziner äußert in diesem Zusammenhang die Idee, dass es die Menschen selbst sind, die mit ihrer Persönlichkeit, ihrem Bewusstsein und Verlangen nach Selbstbestimmung und ihrem persönlichen Umfeld einen sogenannten guten Tod sterben können und dass ein solcher Tod nur in wenigen Fällen Verdienst einer medizinischen Institution ist.

Und der würdevolle Tod, wissen Sie (Sehr im Dialekt betont) würdevoll (.) ja, was ist würdevoll? Wissen Sie, in der Palliativmedizin da schlagen ja nur die ein Prozent Leute auf, wo es irgendwie Probleme gibt, die restlichen sterben irgendwo. Und dieses eine Prozent so hoch zu stilisieren, muss ich ganz ehrlich sagen, ist falsch. Und das wird eigentlich so sein, dass das zum Negativen der Palliativmedizin sein wird, ja? Wir behandeln einfach problematische Fälle, zu Hause, die Angehörigen machen viel mehr, der Patient macht viel mehr selber. Ich glaube das würdevolle Sterben das hängt sehr viel auch mit Autonomie zusammen. Kann jemand würdevoll auch in einer ziemlich würdelosen Umgebung sterben? Das hängt sehr von der Person ab und wie sehr er sich auf den Tod eingestellt hat. Da kann man vielleicht ein bisschen was helfen, aber wir brauchen keine Palliativmedizin um würdevoll zu sterben. (Interview 19, Abs.99, PM) 
In den folgenden Zitaten beispielhaft aufgeführt, kritisieren ein Hausarzt und ein Onkologe die mittlerweile überhöhte Sichtweise auf das Sterben, die mit der Entwicklung von Idealbildern in Bezug auf den Umgang mit dem menschlichen Tod einhergegangen sind. Im Rahmen der Selbstreflexion hinsichtlich ärztlicher Grenzen betont der Hausarzt die weite Dimension des Sterbens, die ein Sterbender und seine Angehörigen für sich selbst verarbeiten und verkraften müssen, unabhängig wie umfangreich und durchdacht ein potentielles medizinisches Konzept im Hintergrund sind.

Der Tod ist ein Skandal. Damit müssen wir leben und müssen das akzeptieren. Man kann dem seine Schärfe nicht nehmen. Und natürlich gibt es dann Rituale, die jede Gesellschaft hat, also wir haben halt unsere Begräbnisrituale, und das ist auch gut so und die möchte ich auch nicht missen und ich möchte auch, dass es eine Grabstätte gibt, auch wenn da nur eine Urne drinnen steht, weil wir so etwas brauchen. Aber das ist einerseits eben, obwohl es so wichtig ist, nur an der Oberfläche. So muss halt jeder selber damit klar kommen (...). (Interview 15, Abs.111, HA)

Der Onkologe betont noch einmal die Gefahr, dass mit der positiv assoziierten Vokabel des würdevollen Sterbens reelle Aspekte ausgeblendet werden können, die in der konkreten Praxis eine bedeutende Rolle spielen können.

Man tut auch immer so als sei der Tod etwas Schönes. Also Tod ist meistens nicht schön, also es kann für manche natürlich tatsächlich eine Erlösung sein, manchmal, will ich gar nicht abstreiten hat es so etwas fast Schönes, wenn jemand sozusagen abgeschlossen hat, aber in den meisten Fällen ist es nicht so. Und viele Menschen, die sterben, die sterben sterben zu jung, die wollen nicht sterben. Und das würde ich gar nicht so schön glorifizieren. Das ist es einfach nicht. Das ist schön reden. (Interview 17, Abs.77, Onkologe)

Eine potentiell $\mathrm{zu}$ positive und als überhöht transportierte Sichtweise auf den menschlichen Tod wird, wie beschrieben, von den meisten praktizierenden Medizinern als problematisch angesehen. Im Rahmen der Diskussionen, inwiefern die individuellen Umgangsformen der Patienten mit den im Rahmen von Idealvorstellungen entwickelten Bildern eines guten Sterbens überhaupt zusammen gebracht werden können, wird insgesamt kritisch betrachtet. So betont eine Palliativmedizinerin beispielhaft, dass die aktuell bestehende Erwartungshaltung, jeder sollte in der Lage sein, offen über das Thema Sterben und Tod und über die Schwierigkeiten, die damit einhergehen, sprechen können, 
nicht zu jedem Patienten passt und dass man als Arzt in der Lage sein muss, sich mit seinen persönlichen Vorstellungen über einen guten Umgang zurückzunehmen.

Also, es gibt Leute, die sterben mit dem Gedanken "Es wird wieder gut." Und wenn das so ist, und ich habe mehrfach, ich kann das nur anbieten. Und wenn jemand das absolut nicht will, dann muss ich das akzeptieren. Nur weil ich glaube, es ist gut, man muss drüber reden, muss das für den Patienten nicht gut sein. (Interview 11, Abs.69, PM_in)

Ein Onkologe betont die Individualität, die jeder Tod mit sich bringt, welche es für ihn unmöglich macht ein einziges als wertvolles erachtetes Bild für den menschlichen Tod zeichnen zu wollen.

Also wir alle wollen würdevoll sterben, klar, und wir wollen auch möglichst ohne Leiden sterben, aber, also ich glaube nicht, dass es dafür nur ein Bild gibt und ich glaube auch, dass jeder Mensch seinen eigenen Weg geht wenn er stirbt. (Interview 9, Abs.83, Onkologe)

In Bezug auf die Idee eines guten Todes, der, wie das Konzept der Palliativmedizin es zu gewährleisten versucht, unter einer guten körperlichen Symptomkontrolle und mit psychologischer Begleitung ablaufen soll, beschreibt ein Hausarzt seine Erfahrungen mit einem tatsächlich guten Tod als eine sehr seltene Ausnahme.

Das ist halt nie (.) Sterben ist halt nie schön. Das sind, äh, das sind Märchen, dass jemand schön stirbt oder gut stirbt, das sind ganz wenige Fälle, wo jemand wirklich dann an Altersschwäche stirbt, ansonsten ist Sterben nie schön. (Interview 15, Abs.55, HA)

Mit Blick auf das Konzept und die Ideale des Fachbereichs Palliativmedizin reflektiert ein Palliativmediziner selbst, dass es auch problematisch sein kann, sich in einer komplexen Situation, wie dem Lebensende eines Menschen zu sehr an ein ideelles Konzept halten zu wollen. Besonders in Bezug auf den Aspekt der Offenheit betont er, das Recht eines Jeden, den eigenen Tod auch in einem Behandlungskontext ausblenden und verdrängen zu dürfen.

Es ist ja auch immer die Gefahr in der Palliativmedizin, dass man ein Schema hat, eine Erwartung hat, ähm, wie das Sterben ablaufen muss und wie sozusagen die Gespräche ablaufen müssen, und wie sozusagen die Zeit 
davor ablaufen muss. Und es gibt so eine Tendenz, äh, eben (.) ja so eine Normierungstendenz kann man fast sagen, ähm, man muss über Sterben uns Tod sprechen, man muss sich möglichst versöhnen mit allen, man muss friedlich sterben und es gibt halt wirklich Patienten, die das vielleicht nicht wollen und zu denen das nicht passt. Und ich glaube ein Mensch hat auch das Recht, wenn er will, tatsächlich das Sterben und den Tod auszublenden. (Interview 20, Abs.47, PM)

Auch mit Blick auf die Arzt-Patienten-Interaktion vor der aktiven Gestaltung des Lebensendes betont ein Hausarzt, dass selbst die Kommunikation über den potentiellen Tod nur sehr überlegt geführt werden sollte:

Ich halte das auch für einen großen Irrtum in dieser ganzen Sterbedebatte, dass manche sich da berufen fühlen, das in den Patienten rein zu drängen. Da muss man vorsichtig sein. (Interview 4, Abs. 17, HA)

Gerade das Auseinandersetzen mit dem eigenen Tod kann, betrachtet man die Arbeiten Anselms, dazu führen, dass der Tod eine Form von Macht über das Leben bekommt. Ein Aspekt, den weder eine gesunde noch eine schwer kranke Person für sich und ihr Leben will (vgl. Anselm 2008). So kann es also auch ein Element bewusster Selbstbestimmung sein, nicht über den eigenen Tod nachdenken und kommunizieren zu wollen und entsprechende Entscheidungen an den Arzt abdelegieren zu wollen.

Bei der generellen Auseinandersetzung mit dem Tod wird zudem seitens der Interviewpartner auf den Aspekt eingegangen, dass man bei der Entwicklung eines potentiell wertvollen Konzepts dazu neigt, von den eigenen Vorstellungen auszugehen. Anhand des empirischen Materials wird zudem deutlich, dass insbesondere Professionelle dazu neigen, ein festes Konzept oder eine Schablone für das Sterben verinnerlicht zu haben, welches sie zu verfolgen versuchen und somit möglicherweise weniger offen für individuelle Probleme und Ängste sind als sie es eigentlich intendieren.

\subsubsection{Rollenkonsistenz der einzelnen Professionen}

Die Überlegung, inwiefern ärztliche Kommunikationsstrategien und Umgangsformen einen Einfluss auf die Autonomie des Patienten haben, steht in einem engen Zusammenhang mit der jeweiligen Beziehung der Personen, die miteinander agieren. Darüber hinaus scheint es, wie das empirische Material zeigt, von wesentlicher Bedeutung zu sein, wie die einzelnen Gesprächspartner in ihrem Umgang von dem eigenen Rollenverständnis 
geprägt werden. Der folgende Abschnitt widmet sich deshalb einigen Dimensionen der Arzt-Patienten-Interaktion und der Überlegung, inwiefern die Selbstwahrnehmung und das Rollenverständnis von Ärzten für den innerkommunikativen Umgang von Bedeutung ist.

Neben der vielfach rezipierten Betonung von Offenheitskonzepten wird häufig ein Distanzieren von Gesprächen über die Grenzen des medizinisch Machbaren oder das Thema Tod beschrieben. In Bezug auf die ärztlichen Handlungsweisen werden Grenzen offensichtlich durch ein gewisses Selbstverständnis geprägt. Dies wird anhand von Aspekten des Heilens und dem Gegenüber stehend der Akzeptanz des Todes, sowie die Kommunikation über das Sterben, besonders greifbar. So wird in dem folgenden exemplarisch aufgeführten Zitat deutlich, dass der Themenbereich Sterben und Tod nicht mit dem eigenen ärztlichen Handeln, sondern mit dem Aufgabenbereich einer anderen Profession assoziiert wird.

Aber dass sie jetzt auf so einer Ebene Tod und was ist danach mit mir sprechen, das kommt eigentlich nie vor. Möchte ich auch nicht. Das ist, das ist dann auch nicht meine Aufgabe. Also, ich bin halt nicht der Pfarrer. Und da hat jeder sein Weltbild und da kann ich ihm auch nicht weiter helfen. Dass unser Leben zu Ende geht, das muss man akzeptieren und ich kann ja nicht, es ist schlimm und es ist so, aber ich kann da nichts ändern. (Interview 15, Abs.91, HA)

Auch das folgende Zitat zeigt ein Handeln, das der Logik des eigenen Wissens folgt und demzufolge durch eine sehr rollenspezifische Argumentation geprägt ist. Das hier zugrunde liegende Wissen und die Selbstwahrnehmung sind eindeutig der Rolle der kurativ tätigen Medizinerin zugehörig.

Also, um ehrlich zu sein, ich delegiere das echt an die Palliativmediziner. Ich sage "Ich bin hier für die Therapien, und ich habe Ihnen hier einen Termin gemacht bei Herrn Y, gehen Sie mal hin, nehmen Sie Ihre Frau mit, wenn sie da ist und, ja, dann können Sie alle anderen Fragen stellen." (Interview 7, Abs.81, Gastroenterologin)

Auch auf ein Nachfragen hin, inwiefern das Gespräch aufgefasst und möglicherweise weitergeführt wird, wenn ein Patient seine Fragen hinsichtlich des Lebensendes wider Erwarten doch an sie adressiert, äußert sich die Ärztin wie folgt: 
Ich will auch das nicht machen müssen, verstehen Sie? Weil ich wirklich der Therapeut bin, wenn die jetzt noch in die andere Sparte rutschen, äh, dann ist das wieder das, was mir mit der Krankenschwester passiert ist [Konflikt nach fachlich formulierter Ansage, das Therapiekonzept von kurativ auf palliativ zu ändern]. Ja, dann denken die ich würde vielleicht nicht mehr alles machen, was man machen kann und deswegen versuche ich das zu trennen. (Interview 7, Abs.83, Gastroenterologin)

In Hinblick auf eine Art Abgrenzung von dem Themenbereich Sterben und Tod schildert ein Palliativmediziner seine Erfahrung mit Kollegen, die sich mit Rekurs auf das ärztliche Selbstbild und das eigene Rollenverständnis eindeutig vom Tod und den kommunikativen Umgang mit diesem distanzieren. Ein Aspekt, der in ähnlicher Formulierung häufig rezipiert wurde.

Also ich kenne da einige Kollegen, so aus der Vergangenheit, einige von denen können dies auch explizit benennen, die Sachen "Der Tod ist mein Feind und ich bin deshalb so ein guter Arzt, weil, ich gehe jeden Tag in die Klinik um den Tod zu besiegen."(.) Und damit ziemlich beeindruckende Sachen im Bereich Intensivmedizin, Hämatologie, Transplantationsmedizin erreicht haben, die aber auch explizit sagen "Wenn es nicht funktioniert, dann will ich am liebsten gar nichts damit tun haben." (Interview 3, Abs.51, PM)

Die systemtheoretische Idee, wonach die Medizin sich über die Unterscheidung gesund oder krank konstituiert, es also primär darum geht, kranke Körper gesund zu machen, schließt in erster Linie automatisch dritte Werte wie gesund, krank und tot aus. Die Aussagen, die die sowohl kurativ als auch palliativ tätigen Mediziner treffen, können demnach nicht nur als kommunikative Strategien zur Umgehung unangenehmer Situationen, wie beispielsweise das offene Gespräch über den Tod, gelesen werden, sondern auch als Aussagen von primär oder intuitiv kurativ orientierten Ärzten, für die erst eine solche Distanzierung oder Verdrängung möglicherweise das weiterarbeiten gemäß der medizinischen Logik erlaubt.

Neben dem beschriebenen Aspekt lässt sich innerhalb des kommunikativen Umgangs zwischen Arzt und Patient ein weiterer interessanter Aspekt aufzeigen. Neben der eindeutigen Befürwortung von Offenheitskonzepten hinsichtlich der Kommunikation über Fragen am Lebensende, scheint parallel dazu die Akzeptanz von Verdrängungsmechanismen eine wesentliche Rolle zu spielen. Insbesondere das im Rahmen einer Ethisierung der 
Medizin angestrebte Offenheitskonzept zur Förderung von Patientenselbstbestimmung lässt an dieser Stelle ein Paradoxon sichtbar werden. Einerseits entspricht die Akzeptanz von Verdrängungsmechanismen dem Respekt vor der Patientenselbstbestimmung, die eben nicht bei jedem Individuum mit dem Wunsch, offen über das Sterben oder über die Grenzen des medizinisch Machbaren kommunizieren zu wollen, einhergeht. Andererseits bringt die entgegengesetzte Überlegung den Gedanken mit sich, dass gerade die Akzeptanz von Verdrängungsmechanismen die Selbstbestimmung des Patienten untergräbt indem für den Verbleib des Lebens entscheidungsrelevante Themen ausgeklammert werden. Betrachtet man nämlich die grundlegende Idee des informed consent, so ist der behandelnde Arzt derjenige, der initial lenkt, welche Themen und Probleme Raum im Arzt-Patienten-Gespräch erhalten. Ein praktizierender Mediziner, der die Zurückhaltung und die Verdrängung seines Patienten von Beginn der Interaktion an akzeptiert, macht demnach den offenen Diskurs über mögliche Schwierigkeiten und Ängste am Lebensende unmöglich.

Trotz der Tatsache, dass sich die große Mehrheit der Interviewpartner für eine offene Kommunikation, insbesondere mit Blick auf schwierige Themen, wie Sterben und Tod, stark macht und im Rahmen der Interviews diesbezüglich sehr konkrete Kommunikationsstrategien beschreibt, scheinen diese in bestimmten Situationen verworfen zu werden. Wie die folgenden Zitate zeigen, scheint das potentielle Gespräch über den Tod, über eine möglicherweise schlechte Prognose und über die vielschichtigen Probleme, die damit einhergehen, oftmals keinen Raum mehr zu erhalten, wenn Patienten bestimmte Formen von Verdrängungsmechanismen signalisieren.

In diesem Sinne betont ein Palliativmediziner, dass die Ignoranz von Verdrängungsmechanismen seines Erachtens nach sogar mit einer Verletzung der Psyche des Patienten einhergehen kann.

I: Akzeptieren Sie es, wenn ein Patient sagt, dass er bestimmte Dinge gar nicht so genau wissen will?

B: Ähm, also grundsätzlich ja. (..) Weder habe ich das Recht noch habe ich die Lust darauf, irgendjemandem weh zu tun mit etwas, was er gar nicht will. (Interview 3, Abs.41, PM)

Ein Onkologe stellt eine Verknüpfung zwischen der Akzeptanz von Verdrängungsmechanismen und der während eines Krankheitsprozesses für inn wichtige Hoffnung auf der 
Patientenseite her. Er plädiert aufgrund dieses möglichen Zusammenhangs eindeutig für die Akzeptanz von Verdrängung.

Man muss aber, also was ich glaube was ganz wichtig ist, Verdrängung akzeptieren. Also, dass ein Patient auch nach einem ersten Gespräch dann wieder die Hoffnung hat "Es wird alles gut." also nicht ständig die Verdrängung des Patienten zu durchbrechen und auch zu akzeptieren, wenn ein Patient signalisiert, das ist relativ selten, dass mir das passiert, dass einer sagt "Lassen Sie mich in Ruhe, ich will das gar nicht so genau wissen mit der Chemotherapie, mit der Prognose." dass auch, ähm, anzuerkennen und dann auch zu akzeptieren. (Interview 9, Abs. 19, Onkologie)

Wie in diesem Kapitel bereits einleitend diskutiert, geht auch ein Palliativmediziner auf die verschiedenen Facetten von Autonomie ein. Betrachtet man also Autonomie als eine Art Garantie für situationsbezogene Handlungsautonomie, so kann diese zum Ausdruck gebracht werden, indem jemand den offenen Diskurs oder, wie in dem folgenden Zitat erkennbar wird, das Nachdenken über den Tod ablehnt.

Wenn wir über Autonomie sprechen, dann muss es auch die Autonomie des Menschen geben, zu sagen "Ich denke über Tod nicht nach." oder Woody Allen hat mal gesagt "Vor dem Tod habe ich keine Angst, aber ich möchte nicht dabei sein." und das ist doch ein ganz klares Zeichen, dass viele dieses auch nicht gestalten wollen. (Interview 21, Abs.51, PM)

Eine Ausnahme, die vielfach thematisiert wurde, stellt jedoch die Situation dar, die sich bei zurückbleibenden Kindern ergibt. In diesem Kontext ist es eindeutig Konsens, die Verdrängungsmechanismen eines sterbenden Erwachsenen aufzubrechen, um die Weiterversorgung der zurückbleibenden Kinder organisieren zu können.

Analog zu den dargestellten Aspekten, Offenheit als Grundstrategie und die damit einhergehenden Probleme, zeigen auch die Ergebnisse zu dem Umgang mit dem Thema Verdrängung, dass dessen Akzeptanz eine wesentliche Rolle zu spielen scheint und sogar als eine Art Kontrapunkt den Offenheitskonzepten gegenüber steht.

Wie bereits diskutiert, ist ein Weiterarbeiten im Sinne der eigenen Handlungslogik möglicherweise erst durch die Akzeptanz von Verdrängung möglich und eben nicht durch eine grenzenlos offene Kommunikation, wie sie beispielsweise in den Idealen der Palliativmedizin thematisiert wird. Die Mediziner folgen somit in ihren Umgangs- und Kommunikationsformen ihrer ärztlichen Handlungslogik und bewegen sich in einer 
eindeutig rollenspezifischen Argumentation, in der die Arztfigur als Heiler und weniger als psychologischer Unterstützer betrachtet wird.

Folgt man dieser Hypothese, so können durch die feste Verwurzelung mit dem eigenen Rollenbild demnach Fragen und Gespräche zu der Thematik Tod und Sterben zum Teil nicht zugelassen werden. Trotz der in der theoretischen Reflexion vorhandenen Bereitschaft für Offenheitskonzepte in der Arzt-Patienten-Interaktion, hält sich die klassische Arztfigur tendenziell an das Bild des Heilers. Die praktizierenden Akteure des Medizinsystems scheinen demnach stärker an ein klassisches Rollenbild gebunden zu sein als man es anhand ihrer erzählten Wahrnehmung zunächst annimmt. Zudem verhält sich die Intention, für die Förderung von Patientenselbstbestimmung zu argumentieren teilweise ambivalent zu dem oftmals parallel bestehenden Bestreben, stets eine offene Kommunikation unterstützen und fördern zu wollen. Schließlich kann sich ein Patient im Rahmen seiner Autonomie dafür entscheiden, bestimmte Themen ausblenden zu wollen und seine Beziehung zu behandelnden Ärzten eindeutig auf medizinische Aspekte beschränken zu wollen. Saake diskutiert diesbezüglich den Aspekt, dass Gesprächsinhalte, die über medizinische Themenbereiche hinausgehen, auch zum Problem werden können. Der behandelnde Arzt geht aufgrund eines Idealbildes mit einer gewissen Erwartungshaltung in das Arzt-Patienten-Gespräch, was Saake zufolge dazu führt, dass die Kommunikation durch den vorher bestehenden Erwartungshorizont eingeschränkt wird (vgl. Saake 2003).

\subsection{Professionsimmanente Spannungen in der medizinischen Praxis}

Hinsichtlich der Verflechtung von Medizinethik und dem medizinischen Praxisgeschehen wird häufig die Kritik formuliert, dass die für die Praxis entwickelten Ideale und Handlungsanweisungen von den Handlungslogiken der Praxis divergieren können. Betrachtet man die selbstreflexive Ebene der Palliativmedizin anhand des empirischen Materials, so wird jedoch ein interessanter Aspekt deutlich. Es scheint demnach neben dem klassischen Spannungsverhältnis zwischen Theorie und Praxis auch innerhalb der Praxis selbst Schwierigkeiten mit den selbst entwickelten Idealvorstellungen zu geben.

\subsubsection{Abgrenzung zum manifesten Selbstbild der Palliativmedizin}

Geht man tiefer auf die Schilderungen und Erfahrungen der Ärzte ein, so zeigt sich vielfach sowohl anhand der erzählten Wahrnehmung der Palliativmediziner als auch anhand der Erfahrungen der primär kurativ tätigen Kollegen, dass sich die Ideale der Palliativmedizin nicht immer umsetzen lassen und dass sich Situationen am Lebensende 
vielfach komplexer darstellen, als man diese in der theoretischen Überlegung zu entwickeln vermag. Auf die sehr vereinzelnd aufkommende Diskussion hin, inwiefern es für die kurativen Bereiche des Medizinsystems relevant ist, dass die Palliativmedizin in der Regel losgelöst von den anderen als eigenständiger Fachbereich arbeitet argumentiert ein Palliativmediziner dafür, dass die Palliativmedizin auf Dauer nicht in der momentan Form weiter bestehen sollte, sondern ihre Ideale langfristig als eine Art Vorbildfunktion in die anderen medizinischen Fachbereiche integrieren sollte.

Wir haben letztendlich sowieso nicht die Strukturen, alle Patienten, die es bräuchten auf einer Palliativstation zu versorgen. Selbst wenn wir sie hätten, wäre es gut, weil dann würde es eine noch abruptere Trennung geben, und letztendlich sollte es eigentlich Ziel sein, dass das know-how der Palliativmedizin und die Haltung, vor allem die Haltung der Palliativmedizin, in den Rest mit einzubringen. Ich meine, wenn jemand fünf Jahre lang eine Tumorerkrankung hat, diese Art von Wertschätzung, Unterstützung, da bleiben, emotionale Nähe, Sicherheit geben, all diese Sachen. Die sollen ja nur nicht nur die letzten zehn Tage für die Patienten da sein. Schwer traumatisiert und in einer Krisensituation ist der Patient ja von Anfang an. Die sollten eigentlich auch die vier Jahre davor irgendwie relevant sein. Von daher ist eigentlich die Idee, dass die Palliativmedizin so eine Art trojanisches Pferd ist, die diese Haltung in den Rest reinbringt und sich im Idealfall in 20 Jahren selbst überflüssig macht. (Interview 3, Abs.81, PM)

\subsubsection{Handlungsideale in der medizinischen Praxis}

Betrachtet man die stets geführte Kontroverse, dass jegliche in der Theorie entwickelten Handlungsanweisungen oder Idealvorstellungen, unabhängig von ihrer Ursprungsdisziplin, das Praxisgeschehen nie vollständig abbilden und erreichen können, so wird anhand der folgenden Zitate ein weiterer interessanter Aspekt deutlich. Nicht nur die in der Theorie formulierten Handlungsanweisungen und Leitlinien, sondern auch die von den Akteuren des Praxisgeschehens selbst entwickelten Ideale scheinen keine ausreichende Nähe zu den komplexen Problemen der Praxis herstellen zu können. Neben der teilweise fehlenden Anwendbarkeit scheinen die Handlungsakteure in bestimmten Situationen sogar überfordert zu sein. Im Zuge der Ethisierung der Medizin wird demnach nicht nur Druck von außen auf das Medizinsystem aufgebaut, sondern auch aus den intrinsischen Vorstellungen und Idealen selbst heraus. 
Mit Blick auf die Intention der Palliativmedizin, einen sogennanten guten Tod ermöglichen zu können und in diesem Zuge als Alternative zu dem Wunsch nach Suizid eine Rolle spielen zu können, äußern sich die Palliativmediziner in den folgenden beispielhaft aufgeführten Zitaten sehr zurückhaltend und selbstkritisch.

Und klar, ich meine die Patienten, die wir hier auf Station haben haben eine durchschnittliche Liegezeit von zehn Tagen, und wir haben eine Mortalitätsrate von dieses Jahr 75\% bis letztes Jahr waren es knapp über 60\%. Das heißt in zehn Tagen, bei jemandem, der im schwerst kranken Zustand hier aufschlägt, aus verschiedenen Gründen, noch zu versuchen, das alles zu eruieren ist (.) und, ähm, einen Prozess anzustoßen, ist vorsichtig gesagt, sehr optimistisch. Also, man wo man sagen muss, also allein, wenn er nicht vor Schmerzen schreit, also allein wenn er irgendwie nicht dauern erbricht, keine Luftnot hat, nachts halbwegs schlafen kann, die Angehörigen wissen, dass sie nur noch kurze Zeit haben und den räumlichen Rahmen kriegen, dass falls etwas besprochen werden muss oder besprochen werden soll, dazu auch noch nie Rahmenbedingungen da sind. Wenn wir das hinkriegen, dann sind wir schon verdammt gut. (Interview 3, Abs.73, PM)

Ähnlich wird die benannte Zurückhaltung auch im Folgenden betont. Mit Blick auf die im Voraus formulierten Idealvorstellungen und Ziele im Umgang mit dem Lebensende scheint es in der Realität um sehr viel kleinschrittigere Ziele zu gehen. Während der große Gedanke der Gestaltung des Lebensendes offensichtlich in der Hintergrund rückt, scheint an dieser Stelle der Umgang mit Krisensituationen am Lebensende vorrangig zu sein.

Aber das, ähm, Patienten, ja (.) das, was ich unter Gestaltung verstehe, das heißt das aktive Vorplanen und Vorsorge treffen, wie soll die Situation, wie soll das drum herum sein, wenn ich sterbe, dass jemand so konkret das plant und auch voraus ahnen kann, das ist in der Realität kein Thema, zumindest in unserem Kontext nicht und (.) da werden wir auch ab und zu mal überrannt vom Tempo der Ereignisse. Dass man das gar nicht so genau planen kann und was wir halt einfach versuchen, was sind die Aufträge oder die Zielvorgaben, die die uns machen, und das ist oft sehr sehr konkret. Aber an dem Erkrankungsbild orientiert. und nicht, äh, was vielleicht den Gestaltungsaspekt ein bisschen trifft ist, wo möchte ich sterben, Krankenhaus oder zu Hause, ne? 
Also das so als ganz rudimentärste Form der Gestaltungswünsche. (Interview 8, Abs.77,PM_in)

Eine andere Palliativmedizinerin betont, dass allein angesichts der Berücksichtigung unterschiedlicher Biographien kein einheitliches Ideal für ein gutes Sterben oder eine gelungenen Gestaltung des Lebensendes existieren kann.

B: Und mancher stirbt auch so wie er gelebt hat und manchmal war es nicht schön, ja? Und trotzdem, man versucht immer so, also ich glaube, dass gerade im Bereich Palliativ oder Hospiz so, dass man auch nicht denken muss, dass man für alle alles lösen kann, ja? Viele Leben bleiben ungelöst.

l: Obwohl es ja manchmal ein wenig von der Palliativmedizin in Anspruch genommen wird, dass sie in der Lage sei einen "guten Tod" gestalten zu können (wird unterbrochen)

B: Können wir nicht. (Interview 16, Abs.63, PM_in)

Es scheint also hinsichtlich der Reflexion über das am Lebensende Realisierbare oftmals um sehr grundlegende zwischenmenschliche Aspekte zu gehen und nicht nur um den Aspekt der guten Gestaltung der Gesamtsituation.

Was wir versuchen ist, dass so hinzubekommen, dass es für die, die weiter leben müssen, nicht so fürchterlich traumatisierend ist, dass es nicht irgendwie mit Erinnerungen verknüpft ist, dass ein Patient schreiend vor Schmerzen im Bett gelegen hat oder, äh, um Atem ringend dann gestorben ist, das versuchen wir durch adäquate medizinische Therapie so einigermaßen hinzubekommen, aber dass der Abschied schwer ist, auch wen wir das Gefühl haben, das war jetzt eine super Symptom kontrollierte Sache, ähm, da kommen wir nicht drum herum. (Interview 8, Abs.91, PM_in)

Während der intuitiv selbstverständliche Schmerzaspekt in der abstrahierten Diskussion um die Gestaltung des Lebensende oftmals nicht erwähnt wird, wird er an dieser Stelle explizit betont. Es gibt also eine prinzipiell wertvolle Idealvorstellungen hinsichtlich des Umgangs mit dem Lebensende, es bestehen im Rahmen dessen jedoch deutliche Grenzen. Wie mehrfach erwähnt beziehen sich diese Grenzen sowohl auf die Symptomkontrolle als auch auf die Fähigkeit, die Situation psychisch verkraften zu können. 
Bei der Entwicklung einer Idealvorstellung für den guten Tod scheint es also überstilisierte und nicht mit den realen Schwierigkeiten abgestimmte Bilder zu geben. Betrachtet man die Probleme, die anhand des empirischen Materials sichtbar werden, steht dieses Ideal exemplarisch dafür, dass es problematisch sein kann, aus einer normativen Vorstellung heraus Ideale zu entwickeln, welche die Betroffenen möglicherweise überfordern und vielleicht gar nicht für sich annehmen können. Indem man der Medizin, insbesondere einem so vulnerablen Teilbereich wie der Palliativmedizin, Dinge zuweist, die sie möglicherweise nicht erfüllen kann, produziert man somit möglicherweise nicht nur Schwierigkeiten für die Arzt-Patienten-Interaktion, sondern auch für die Integrität des Fachbereichs selbst. Deutlich werden also professionsimmanente Spannungen, die explizit nicht von außen auferlegt und provoziert zu sein scheinen. 


\section{Diskussion}

Vor dem Hintergrund, dass sich der Problemzugriff der vorliegenden Arbeit auf zwei Ebenen abspielt, nämlich auf einer ethischen und einer operationalen, ist hinsichtlich der Analyse zu diskutieren, inwiefern die beiden Säulen Ethik und Empirie generell miteinander zu verzahnen sind. Ein wichtiger Fokus ist in diesem Zusammenhang die Frage, inwiefern sich empirische Ergebnisse prinzipiell in medizinethische Überlegungen integrieren lassen. Die Verknüpfung zwischen den normativen Anteilen der Medizinethik und der deskriptiven empirischen Forschung andererseits hat das Potential, den Diskurs durch Praxisnähe zu bereichern: Ein Aspekt, der von den unterschiedlichen Fakultäten zunehmend eingefordert wird. Das Verhältnis von normativen Elementen der Medizinethik und deskriptiver Empirie wirft jedoch auch eine Reihe von ungeklärten Fragen auf, insbesondere hinsichtlich der methodologischen Spannung zwischen ethischen und empirischen Dimensionen und was diese in Zukunft für die Forschung der Medizinethik bedeuten könnte (vgl. Schicktanz und Schildmann 2009). Schicktanz und Schildmann schlagen diesbezüglich eine an die existierende sozialwissenschaftliche Diskussion heranreichende Methodenreflexion vor, welche die Standards der empirischen Forschung integriert und bei der Interpretation der Ergebnisse berücksichtigt wird (ebd.). Unter Berücksichtigung des beschriebenen Aspekts wurde bewusst die Herangehensweise verschiedener sozialwissenschaftlicher Autoren an die qualitative Forschung betrachtet und von der Durchführung der Interviews bis hin zu der Analyse des empirischen Materials eng mit erfahrenen Kollegen zusammengearbeitet.

Hinsichtlich der in der vorliegenden Arbeit verwendeten Methode der qualitativen Interviewstudie ist anzumerken, dass diese für das Feld der medizinischen Forschung noch verhältnismäßig fremd ist. Im Gegensatz dazu sind quantitative Arbeiten, teilweise auch mit sozialpsychologischem Hintergrund, den Experten des Fachbereichs Medizin vertrauter.

Wie in Kapitel 8. umfassend dargelegt, eignet sich das Experteninterview zur Exploration des Unbekannten. Da die aktuelle Forschung gezeigt hat, dass in diesem Themenfeld Daten fehlen, wurde für das vorliegende Forschungsanliegen die Methodik des Leitfaden gestützten Experteninterviews gewählt.

Mit Blick auf die konkreten Inhalte des Interviewleitfadens ist anzumerken, dass die beleuchteten Begriffe, wie beispielsweise Autonomie und Würde im ersten Schritt von der Autorin aufgeführt wurden. Erst daraufhin erfolgte die Reflexion durch die Interviewpartner selbst. 
Ein weiterer Aspekt, der in diesem Zusammenhang Erwähnung finden sollte, ist die Tatsache, dass Überlegungen und Antworten in der Interviewsituation meist spontan erfolgen. Das bedeutet, dass die Interviewten teilweise auf komplexe Fragen antworten müssen, über die sie sich bis zu dem Moment des Interviews möglicherweise noch keine Gedanken gemacht haben. Mit dem bewussten Ziel, die Handlungswirklichkeit der Interviewten zu rekonstruieren, wurde empirisch an der Alltagswelt der Interviewpartner angesetzt. Bei dem empirischen Material handelt es sich also stets um eine erzählte Wahrnehmung. Demzufolge ist keinerlei Nachweis möglich, ob die Befragten wirklich in der Form handeln würden, wie sie es im Rahmen des Interviews schildern.

Um einen Interpretationsbias von Seiten der Autorin zu verhindern wurde, wie in Kapitel 8. bereits aufgeführt, im Verlauf des Interviews frei mit dem Leitfaden gearbeitet. Durch die weitgehende Eigenpräsentation der Interviewpartner wurde zudem ein Oktroyieren künstlicher Aspekte weitgehend vermieden und ein notwendiges Maß an Offenheit erreicht. Die Datengewinnung erfolgte dabei als kommunikativer Prozess. Die Analyse und Deutung des empirischen Materials erfolgte schrittweise und in einem ständigen reflexiven Bezug, sodass im Rahmen des Forschungsprozesses Themen und Aspekte generiert wurden, die im Vorfeld von der Autorin nicht erwartet wurden. Außerdem erfolgte in regelmäßigen Abständen eine zweite Analyse durch erfahrene Sozialwissenschaftlerinnen.

Losgelöst von der Methodik soll eine weitere Überlegung aufgeführt werden, die den Entwurf von Idealen und Handlungsanweisungen für die Praxis betrifft. Prinzipiell wird von den Akteuren des Praxisgeschehens häufig die Kritik geäußert, dass die Handlungsanweisungen der Theorie zu wenig Berührung mit der Realität haben und meist nicht handlungsrelevant sind. Im Rahmen der vorliegenden Arbeit, die sich den genannten verschiedenen Ebenen widmet, wird jedoch deutlich, dass sich derartige Konflikte auch intern sowohl in den Disziplinen der Praxis als auch in der Theorie ergeben können. So entwickelt die Palliativmedizin beispielsweise Idealvorstellungen und Schablonen für das Lebensende, die sie selbst nicht ausreichend erfüllen kann. Vergleichbar mit diesem Aspekt verhandelt die Medizinethik Narrative, die fern des Praxisgeschehens sind. Dabei professionalisiert sie sich so sehr, dass sie das Problem bekommt, selbst Teil des Konflikts zu werden, den sie erörtert.

Die wichtigste Hypothese, die aus der empirisch-ethischen Reflexion bezüglich des Verwirklichungshorizontes von Autonomie hervorgegangen ist, beschreibt den Zusammenhang von professionsimmanenten Handlungslogiken und die möglicherweise damit einhergehenden Einschränkungen von Autonomie. Anhand des empirischen Materials wurde deutlich, dass die Deutungsmacht über kommunizierbare sowie nicht- 
kommunizierbare Aspekte bei Fragen am Lebensende eindeutig auf Seiten des Arztes liegt. Darüber hinaus hat sich gezeigt, dass aus einer zu offenen Kommunikation über die Grenzen des medizinisch Machtbaren oder den potentiellen Tod Konflikte entstehen können. Die Gesprächspartner stoßen zum Teil beide an ihre Grenzen und registrieren unter anderem durch eine negative Resonanz, besonders auf Seiten des Patienten, dass ein Weiterarbeiten gemäß der eigenen, bisher bestehenden Handlungslogik erst durch die Akzeptanz von Verdrängungsmechanismen möglich ist. Und eben nicht über eine bedingungslos offene Kommunikation, die klar mit der Förderung von Patientenautonomie assoziiert wird.

Hypothetisch kann angenommen werden, dass dahinter die systemtheoretische Idee steht, nach der die Medizin sich über die Unterscheidung gesund oder krank konstituiert, es also primär darum geht, kranke Körper gesund zu machen. Dieser Aspekt schließt in erster Linie automatisch dritte Werte, wie gesund, krank und tot aus. Die Aussagen, die die sowohl kurativ als auch palliativ tätigen Mediziner treffen, können demnach nicht nur als kommunikative Strategien zur Umgehung unangenehmer Situationen, wie beispielsweise das offene Gespräch über den Tod, gelesen werden, sondern auch als Aussagen von primär oder intuitiv kurativ orientierten Ärzten, für die erst eine solche Distanzierung oder Verdrängung möglicherweise das weiterarbeiten gemäß der medizinischen Logik erlaubt. Der Aspekt der Rollenkonsistenz findet sich auch in einer qualitativen Studie von Körtner und Inthorn, in der von ärztlicher Seite aus vielfach der Aspekt betont wird, dass die „Zielsetzung des Arztberufes vor allem darin gesehen werde, Leben zu retten“ (Körtner und Inthorn et al. 2009, S. 20). Folgt man den Ergebnissen der Studie sehen sich die Ärzte als „Experten für das Leben“ (ebd., S. 20). Im Rahmen dessen wird das Leben vor allem aus der Perspektive der Naturwissenschaften betrachtet, indem der Tod als das Ende der Biologie gedeutet wird (ebd.).

Betrachtet man die genannten Aspekte, so zeigt sich erneut die Problematik der fehlenden Verzahnung der Disziplinen. Es existiert jedoch nicht ausschließlich die fehlende Praxisreflexion in der Theorie, sondern parallel dazu genauso ein Theoriedefizit in der medizinischen Praxis. Eine Analysegrundlage für an diese Arbeit anknüpfende Untersuchungen sollte demnach eine tiefergehende Untersuchung der Rollenkonsistenz der ärztlichen Profession mit sich bringen. Damit einhergehen sollte eine Reflexion über dessen Konsequenz in der Praxis sowie über den zukünftigen Umgang mit Rollenvorstellungen im Rahmen der medizinischen Ausbildung.

Abschließend soll ein Blick auf die normativen Elemente von Medizinethik und dem Fachbereich Palliativmedizn erfolgen. Im Verlauf des gesamten Forschungsvorhabens 
geht es um den Verwirklichungshorizont des nahezu unumstrittenen Prinzips der Autonomie und den Umgang mit Idealen, die das Leben und das Sterben des Einzelnen betreffen. Diese normativen Elemente müssen jedoch nicht nur für sich stehend, sondern auch in Bezug auf gewisse Zuständigkeitsansprüche verhandelt und betrachtet werden. Schließlich bringt die Zuständigkeit für Patientenautonomie oder für den medizinischen Umgang mit dem Lebensende eine politische Dimension, wenn nicht sogar eine Form von Systemmacht mit sich. In diesem Sinne existiert beispielsweise ein Diskurs, in dem die Palliativmedizin als eine Alternative zum ärztliche assistierten Suizid diskutiert wird. 


\section{Fazit}

In den aktuellen Debatten - sowohl in der Fachliteratur als auch in dem medialen Diskurs herrscht Einigkeit darüber, dass Patientenautonomie fördernde Konzepte besonders stark gemacht werden sollen. In diesem Zuge hat das Autonomieprinzip beispielsweise in den Idealen der Palliativmedizin im Rahmen des ganzheitlichen und multidisziplinären Behandlunsskonzeptes eine zentrale Bedeutung erlangt. Es gilt jedoch zu hinterfragen, wie man mit dem Prinzip der Autonomie in Zukunft umgehen will und wie man die handlungsrelevante Umsetzung eines theoretisch wertvollen Korrektivs anstrebt.

Mit Blick auf die Spannungen, die anhand des empirischen Materials deutlich werden, und der Intention, diese als konstruktiven Beitrag zu betrachten, lässt sich ein Fazit auf drei verschiedenen Ebenen formulieren:

Erstens zeigt sich bei der Betrachtung des Praxisgeschehens, dass die dort hineingetragenen Ideale von den offensichtlich bestehenden Handlungslogiken der Praxis selbst divergieren können. Besonderes deutlich wird dieser Aspekt bei Ärzten, die dem Imperativ einer Ethisierung der Medizin folgen und die Grenzen des medizinisch Machbaren offen thematisieren. In diesem Zuge wurden vielfach schlechte Erfahrungen beschrieben, wenn das Thema Sterben und Tod im Rahmen der Arzt-Patienten-Interaktion zu offensiv angesprochen wurde. Die Gesprächspartner stoßen, wie anhand des empirischen Materials greifbar wurde, beide an ihre Grenzen. Insbesondere durch eine negative Resonanz auf Seiten des Patienten wird registriert, dass ein Weiterarbeiten gemäß der eigenen bisher bestehenden Handlungslogik erst durch die Akzeptanz von Verdrängungsmechanismen möglich ist und eben nicht über eine bedingungslos offene Kommunikation. Die praktizierenden Akteure des Medizinsystems scheinen demnach stärker an ein klassisches Rollenbild gebunden zu sein als man es anhand ihrer erzählten Wahrnehmung zunächst annimmt.

Zweitens scheint die Verantwortung der Experten aus Medizinethik und Palliativmedizin von großer Relevanz zu sein. Vor dem Hintergrund, was Ethik als eine Art Korrektiv vermitteln will, muss es demnach zunehmend Diskursinhalt sein, ob die fachlich entwickelten normativen Vorstellungen für die Akteure des Praxisgeschehens nicht nur wertvoll, sondern auch problematisch sein können. Parallel dazu fehlt zusätzlich eine reflexive Ebene zur Lösung der diskutierten professionsimmanenten Spannungen, die auch intern für das medizinische Praxisgeschehen problematisch werden können.

Drittens sollte die Überlegung angestellt werden, was aktuell in der Öffentlichkeit geschieht und ob der Diskurs zur Realisierbarkeit des Autonomieprinzips für eine weitere Ebene der Problematik geöffnet werden muss. Der Grund hierfür findet sich darin, dass die öffentliche Debatte das Sterben gegenwärtig in einer derart überhöhten Art und Weise 
betrachtet, dass ein „reales Sterben“ zum Teil wieder tabuisiert wird. Wie bereits anhand des empirischen Materials gezeigt wurde, ist jedoch nicht nur ein Überhöhen des Sterbens problematisch. Prinzipiell ist auch die Entwicklung von Idealen oder Schablonen für einen sogenannten guten Tod sehr ambivalent. Im Zuge dessen entsteht eine Dynamik, die mit einer gewissen Normierungstendenz von teilweise unrealistischen Vorstellungen einhergeht und die dazu neigt, das Sterben pauschal zu glorifizieren. Mit einer initial positiv assoziierten Intention läuft man somit nicht nur Gefahr, dass sich das Abarbeiten an dem Ideal des guten Sterbens sowohl für den Sterbenden selbst als auch für seine Angehörigen als desillusionierend und mühsam erweisen kann, sondern dass im Zuge dessen sogar Handlungsspielräume eingeschränkt werden. Ein Punkt, der wiederum in ein Spannungsverhältnis zur Autonomie geraten kann.

Insgesamt scheint es in Hinblick auf die im Verlauf diskutierten Spannungen eine fehlende Verzahnung zwischen der fachlichen Ausrichtung der Palliativmedizin, den medizinethischen Überlegungen und den Reflexionen in der medizinischen Praxis zu geben. Bei dem Ziel, diese Verzahnung anzustreben, dürfen die theoretischen Disziplinen jedoch nicht zu radikal in dem Publizieren ihrer Ideale werden. Daran anknüpfend muss für die Akteure der Praxis eine Art Imperativ der Eigenverantwortung formuliert werden. Dieser muss die entwickelten Ideale und die Reflexion, die die Medizinethik leistet, integrieren. Zudem sollte stets der Anspruch erhoben werden, die praktische Debatte zunächst zu verstehen. Erst in einem nächsten Schritt können mit dem Wissen über die Logik des Praxisgeschehens neue reflexive Räume erschaffen werden, die die Grenzen der angenommenen Verantwortungsbereiche ausleuchten. Existiert unter dem Deckmantel der Autonomie nur noch der Verantwortungsbereich des Patienten, läuft ein theoretisches Korrektiv wie das Prinzip der Autonomie Gefahr, die Akteure des Praxisgeschehens zu überfordern. Vor diesem Hintergrund sollte darüber hinaus reflektiert werden, ob es gesellschaftliche Konsequenzen aus einer Überbetonung von Autonomie geben kann.

Abschließend muss festgehalten werden, dass die explorativ ermittelten Spannungsverhältnisse im Rahmen des Verwirklichungshorizontes von Autonomie bisher nicht über sozial konstruiertes Wissen hinausgehen. Der Versuch, dieses Wissen normativ zu strukturieren sollte mit Hilfe ergänzender Arbeiten und interdisziplinärer Anschlussprojekte weiter untersucht werden. Insbesondere mit Blick auf den Kernaspekt der Ergebnisse, der den Zusammenhang von professionsimmanenten Handlungslogiken und die möglicherweise damit einhergehenden Einschränkungen von Autonomie beschreibt, können die vorliegenden Analysen eine Grundlage für anknüpfende Arbeiten darstellen. 


\section{Zusammenfassung}

Die Autonomie des Patienten, verstanden als eine Form von Entscheidungsfreiheit und Handlungsfähigkeit, stellt aktuell den zentralen Bezugspunkt des Argumentierens in der Medizinethik dar. Das dazu zählende Recht, selbst über den eigenen Körper zu entscheiden, spielt dabei in der medizinischen Praxis und bei Fragen, die das Lebensende des Menschen betreffen, eine entscheidende Rolle. Autonomie ist indes sowohl positiv assoziierter Mittelpunkt als auch Konfliktpunkt der aktuell vorherrschenden Diskurse der Medizinethik. In Hinblick auf die medizinische Praxis existiert zudem kaum ein vergleichbares Prinzip, das einen ähnlich prägenden Einfluss auf die Arzt-PatientenInteraktion aufweist. Dies gilt sowohl für alltagsrelevante Entscheidungen der medizinischen Praxis als auch für die großen Kontroversen der Medizinethik. Die argumentative Fokussierung auf das Prinzip der Autonomie und die damit einhergehende Wahrnehmung des Prinzips als eine Art Korrektiv ruft jedoch auch Kritik hervor. Insbesondere hinsichtlich der Überlegung, wie fern sich theoretischer Begriff und Praxisgeschehen in der Realität des Medizinsystems sind. Insgesamt hat die Komplexität medizinethischer Probleme wie auch der stets politische und kulturelle Charakter medizinethischer Kontroversen somit dazu geführt, dass sich das einst klare Profil des Autonomieprinzips in sehr unterschiedlichen Diskurslinien - die sich unter dem Begriff der relationalen Autonomie zusammenfassen lassen - weiterentwickelt hat.

Trotz dieser ausdifferenzierten Weiterentwicklung scheint jedoch - mit Blick auf das Praxisgeschehen des Medizinsystems - eine letzte Ebene der Reflexion zu fehlen. An genau diesem Punkt setzt das vorliegende Forschungsvorhaben an. Auf dem Boden einer empirisch-ethischen Reflexion wird die konkrete Frage nach der Realisierbarkeit des Autonomieprinzips aufgeworfen. Indes wird nach der Daseinsberechtigung des Autonomieprinzips als eine Art Korrektiv für das menschliche Handeln und Entscheiden bei Fragen am Lebensende gefragt.

Für die vorliegenden Untersuchungen wurde die sozialwissenschaftliche Methodik der qualitativen Interviewstudie gewählt. Im Konkreten bildet dabei das Leitfaden-gestützte Experteninterview den methodischen Hintergrund des vorliegenden Forschungsvorhabens. Neben dem Abgleich mit dem Autonomiediskurs, wie er sich in der Medizinethik vollzogen hat, ist das Autonomieprinzip, wie es als Ideal in der Palliativmedizin theoretisch entworfen wurde, wesentliche Analysegrundlage. Im Rahmen dessen wurde herausgearbeitet, wie sich das Prinzip der Autonomie aus der Perspektive von Palliativmedizinern und kurativ tätigen Medizinern in deren Handlungswirklichkeit darstellt. 
Um nun also eine Verzahnung zwischen Theorie und Praxis zu gewährleisten, spielt sich das vorliegende Forschungsanliegen auf zwei unterschiedlichen Ebenen ab. Die Arbeit folgt im Rahmen dessen einem operationalen und einem ethischen Problemzugriff.

Der ethische Zugriff beinhaltet das Autonomieprinzip als normatives Prinzip der Medizinethik sowie Ausarbeitungen zu dem Begriff der Würde, der in den aktuellen Diskursen eng mit Überlegungen zum menschlichen Handeln und Entscheiden bei Fragen am Lebensende verknüpft ist. Der operationale Zugang stellt mit seinem Fokus auf Kommunikationsstrategien den Praxisbezug her und begründet das methodische Vorgehen des Forschungsvorhabens. Darüber hinaus verknüpft die vorliegenden Arbeit normative Elemente der Medizinethik und deskriptive Elemente empirischer Forschung. Die Intention, eine Verzahnung zwischen Theorie und Praxis herzustellen, wird dadurch gewährleistet.

In Zusammenschau der Ergebnisse lassen sich anhand des empirischen Materials deutliche Spannungen aufzeigen. Auf dem Boden der Frage nach der Realisierbarkeit des Autonomieprinzips lässt sich abschließend ein Fazit auf drei Ebenen formulieren:

Erstens zeigt sich bei der Betrachtung des Praxisgeschehens, dass die dort hineingetragenen Ideale von den offensichtlich bestehenden Handlungslogiken der Praxis selbst divergieren können. Besonderes deutlich wird dieser Aspekt bei Ärzten, die dem Imperativ einer Ethisierung der Medizin folgen und die Grenzen des medizinisch Machbaren offen thematisieren. In diesem Zuge wurden vielfach schlechte Erfahrungen beschrieben, wenn das Thema Sterben und Tod im Rahmen der Arzt-Patienten-Interaktion zu offensiv angesprochen wurde. Die Gesprächspartner stoßen beide an ihre Grenzen. Insbesondere durch eine negative Resonanz auf Seiten des Patienten wird registriert, dass ein Weiterarbeiten gemäß der eigenen bisher bestehenden Handlungslogik erst durch die Akzeptanz von Verdrängungsmechanismen möglich ist und eben nicht über eine bedingungslos offene Kommunikation. Die praktizierenden Akteure des Medizinsystems scheinen demnach stärker an ein klassisches Rollenbild gebunden zu sein als man es anhand ihrer erzählten Wahrnehmung zunächst annimmt.

Zweitens scheint die Verantwortung der Experten aus Medizinethik und Palliativmedizin von großer Relevanz zu sein. Vor dem Hintergrund, was Ethik als eine Art Korrektiv vermitteln will, muss es demnach zunehmend Diskursinhalt sein, ob die fachlich entwickelten normativen Vorstellungen für die Akteure des Praxisgeschehens nicht nur wertvoll, sondern auch problematisch sein können. Parallel dazu fehlt zusätzlich eine reflexive Ebene zur Lösung von professionsimmanenten Spannungen, die auch intern für das medizinische Praxisgeschehen problematisch werden können. 
Drittens sollte die Überlegung angestellt werden, was aktuell in der Öffentlichkeit geschieht und ob der Diskurs zur Realisierbarkeit des Autonomieprinzips für eine weitere Ebene der Problematik geöffnet werden muss. Der Grund hierfür findet sich darin, dass die öffentliche Debatte das Sterben gegenwärtig in einer derart überhöhten Art und Weise betrachtet, dass ein „reales Sterben“ zum Teil wieder tabuisiert wird. Darüber hinaus ist jedoch auch die Entwicklung von Idealen oder Schablonen für einen sogenannten guten Tod sehr ambivalent. Im Zuge dessen entsteht eine Dynamik, die mit einer gewissen Normierungstendenz von teilweise unrealistischen Vorstellungen einhergeht und die dazu neigt, das Sterben pauschal zu glorifizieren. Mit einer initial positiv assoziierten Intention läuft man somit nicht nur Gefahr, dass sich das Abarbeiten an dem Ideal des guten Sterbens sowohl für den Sterbenden selbst als auch für seine Angehörigen als desillusionierend und mühsam erweisen kann, sondern dass im Zuge dessen sogar Handlungsspielräume eingeschränkt werden. Ein Punkt, der wiederum in ein Spannungsverhältnis zur Autonomie geraten kann. 
13. Literaturverzeichnis

Aichmüller-Lietzmann G: Palliativmedizin in der Praxis - dargestellt am Beispiel der Palliativstation des Johannes-Hospizes in München, Lit Verlag, Münster, 1998

Anselm R (2008): Wir müssen darüber reden. Patientenverfügungen als Kommunikationsinstrumente. Ethik Med 20, 191-200

Anselm R (2002): Gestützte Selbstbestimmung. Perspektiven einer Ethik der Freiheit trotz körperlicher Abhängigkeit. In: Bartmann P/ Hübner I (Hrsg.): Patientenselbstbestimmung. Paradigmenwechsel und Herausforderung im Gesundheitswesen, Neukirchen, 71-84

Anselm R (2011): „Auch die Tatsache, dass man nicht alles weiß, ist eine Bedingung für das je eigenen Leben“ (Bernhard Williams). Evangelisch-theologische Überlegungen über Schicksal, Zukunftsoffenheit und prädikative Medizin am Anfang des Lebens. In: Maio G(Hrsg.): Abschaffung des Schicksals? Menschsein zwischen Gegebenheit des Lebens und medizintechnischer Gestaltbarkeit. Freiburg, Herder, 350-367

Anselm R, van Oorschot B: Mitgestalten am Lebensende - Handeln und Behandeln Sterbenskranker. Vandenhoeck und Ruprecht, Göttingen, 2007

Anselm R, Butz U (2016): Vertrauen in der Organisation Krankenhaus - wie lässt sich das Nicht-Organisierbare organisieren? In: Autonomie und Vertrauen. Schlüsselbegriffe der modernen Medizin. Springer VS, Wiesbaden, 133-162

Baile WF et al. (2000): SPIKES-A Six-Step Protocol for Delivering Bad News: Application to the Patient with Cancer. The Oncologist $\underline{5}$ (4), 302-311

Beauchamp T, Childress JF (1979): Principles of Biomedical Ethics, Oxford University Press, Oxford 1979

Beauchamp T, Childress JF (1994): Principles of Biomedical Ethics. 4. Auflage; Oxford University Press, Oxford 1994 
Beck D, Kettler D (2001): Symptomokontrolle in der Palliativmedizin. Der Schmerz. 15, 320-332

Becker G , Xander C (2008): Partizipative Entscheidung statt Autonomie in der Palliativmedizin. In: Illhard FJ (Hrsg.) Die ausgeblendete Seite der Autonomie, LIT Verlag Dr. W. Hopf, Berlin, 47-57

Beckmann JP (1998): Patientenverfügungen: Autonomie und Selbstbestimmung vor dem Hintergrund eines im Wandel begriffenen Arzt-Patienten-Verhältnisses. Zeitschrift für medizinische Ethik, 44, 143-156

Beckmann JP (2002): Ethische Fragen in der Palliativmedizin. In: Aulbert E et al.(Hrsg.), Palliativmedizin als Ausdruck gesellschaftlicher Verantwortung, Schattauer Verlag, Stuttgart, 113-122

Behmann M et al. (2009): Palliative care in Germany from a public health perspective: qualitative expert interviews, BMC Research Notes, $\underline{2}: 116$

Borasio GD (2012): Über das Sterben. Was wir wissen, was wir tun können, wie wir uns darauf einstellen. Verlag C.H. Beck, München

Brauer D, Lipp V (2016): Autonomie und Familie in der medizinischen Entscheidungssituation. In: Autonomie und Vertrauen. Schlüsselbegriffe der modernen Medizin. Springer VS, Wiesbaden, 201-237

Bührmann A (2004): Rezension: Alexander Bogner, Beate Littig \& Wolfgang Menz (Hrsg.) (2002). Das Experteninterview. Theorie, Methode, Anwendung. Forum Qualitative Sozialforschung/ Forum: Qualitative Social Research, 5

Bundesgesetzbuch (BGB) (2008): http://dip21.bundestag.de/dip21/btd/16/084/1608442.pdf (letzter Zugriff am 25.03.2015)

Butzlaff M et al. (2003): "Shared Decision Making": Der Patient im Mittelpunkt von Gesundheitswesen und Praxisalltag. In: Böcken J et al. (Hrsg.): Gesundheitsmonitor 2003, Gütersloh, 41-55 
Cella DF et al. (1995): Reliability and validity of the Functional Assessment of Cancer Therapy-Lung (FACT-L) quality of life instrument. Lung Cancer 12:199-220

Chochinov HM (2002): Dignity-conserving care - a new model for palliative care: helping the patient feel valued, JAMA 287 (17), 2253-2260

Chochinov HM et al. (2002a): Dignity in the terminally ill: a developing empirical model, Soc Sci Med 54 (3), 433-443

Christman J (2011): Autonomy in Moral and Political Philosophy. In: The Stanford Encyclopedia of Philosophy (Spring 2011 Edition), hg. von N.Z. Edward, http:// plato.stanford.edu/archives/spr2011/entries/autonomy-moral/ (letzter Zugriff: 12.03.2016)

Deutsche Gesellschaft für Palliativmedizin (DGP) (2015): http://www.dgpalliativmedizin.de/ allgemein/sapv.html (letzter Zugriff am 24.09.2016)

Donchin A (2001): Understanding Autonomy Relationally: Toward a Reconfiguration of Bioethical Principles. J Med Philos $\underline{26}$ (4), 365-386

Duttge G (2011): Vertrauen am Lebensende durch Recht? In: Höver G et al (Hrsg.): Sterbebegleitung: Vertrauenssache. Würzburg, Verlag Königshausen und Neumann, 143-174

Duttge $G$ et al. (2016): Vertrauen durch Recht?. In: Autonomie und Vertrauen. Schlüsselbegriffe der modernen Medizin. Springer VS, Wiesbaden, 239-291

Düwell M, Hübenthal C, Werner MH (2002): Handbuch Ethik, J.B. Metzler Verlag, Stuttgart

Feinberg J (1986): Harm to self. The moral limits of the criminal law, Oxford University Press

Feuerstein G: Biopolitische Paradoxien der Patientenautonomie. In: Wiesemann C, Simon A (Hrsg.): Patientenautonomie. Theoretische Grundlagen, Praktische Anwendungen. Mentis, Münster, 2013, 244-256 
Franck G, Sitte T (2013): Wahrnehmung und Kommunikation. In: Thöns M, Sitte T. (Hrsg.), Repetetorium Palliativmedizin, Springer Verlag, Berlin

Freidson E (1975): Dominanz der Experten. Zur sozialen Struktur medizinischer Versorgung. Urban \& Schwarzenberg, München

Galuschko M, Voltz R (2011): Durchführung qualitativer Interviews. Zeitschrift für Palliativmedizin. 12, 54-56

Geisler LS (2004): Patientenautonomie - eine kritische Begriffsbestimmung. Dtsch Med Wochenschr 129, 453-456

Großmann A (2004): Würde. In: Ritter J et al. (Hrsg.), Historisches Wörterbuch der Philosophie, Schwabe AG, Basel, 1088-1094

Hack TF et al. (2002): Defining dignity in terminally ill in cancer patients: a factor-analytic Approach. Psychooncology 13 (10), 700-708

Herman K et al. (2010): Versorgung von Palliativpatienten in der Hausarztpraxis Übereinstimmung der Einschätzung der Betreuung aus Arzt- und Patientensicht der Palliative Care Outcome Scale (POS), Palliativmedizin, 11, 26-33

Hopf C (2003): Qualitative Interviews - ein Überblick. In: Flick U et al. (Hrsg.): Qualitative Forschung. Rowohlt Taschenbuch Verlag, Reinbeck bei Hamburg, 349-360

Huber H (2010): Kant - Grundlegung zur Metaphysik der Sitten. In: Huber, Herbert (Hrsg.): Klassische Werke zur philosophischen Ethik. Studienbuch für Philosophie- und Ethiklehrer, Freiburg, 171-198

Husebo S, Klaschik E (2009): Palliativmedizin Grundlagen und Praxis, Springer Medizin Verlag, Heidelberg

Illhardt FJ (2008): Ungelöste Probleme der Autonomie: Unterwegs zu einem neuen Konzept. In: Illhard FJ (Hrsg.) Die ausgeblendete Seite der Autonomie, LIT Verlag Dr. W. Hopf, Berlin, 189-203 
Jaspers K (1953): Der Arzt im technischen Zeitalter, Thieme, Stuttgart

Jordan I et al. (2016): Familien und Patientenorganisationen als kollektive Akteure in der Bioethik: vernachlässigt und unterschätzt? In: Autonomie und Vertrauen. Schlüsselbegriffe der modernen Medizin, Springer VS, Wiesbaden, 163-191

Kant I (2007): Grundlegung zur Metaphysik der Sitten, Suhrkamp Verlag, Paderborn

Kant I (1968): Grundlegung zur Metaphysik der Sitten, Walter de Gruyter und Co Verlag, Berlin

Karpowicz P et al. (2009): Human/Non-Human Chimeras, The Human Dignity Argument in The Stanford Encyclopedia of Philosophy (2009 Edition), Edward N. Zalta (ed.), URL http://plato.stanford.edu/archives/spr2011/entries/chimeras/ (letzter Zugriff am 12.03.2016)

Kaufmann SR (2006): ... and a time to die - How American Hospitals Shape the End of Life, The University of Chicago Press

Kettler D: Selbstbestimmung am Lebensende. Sterben wo und wie? Palliativmedizin - die vierte Dimension in der modernen Gesundheitsversorgung. In: Kettler D, Simon A, Anselm A, Volker L, Duttge G (Hrsg.), Selbstbestimmung am Lebensende. Ringvorlesung im Wintersemester 2005/ 06, Göttingen, 2006, 150-160

Klaschik E (2002): Palliativmedizin - integraler Bestandteil der Hochleistungsmedizin in Aulbert E et al. (Hrsg.), Palliativmedizin als Ausdruck gesellschaftlicher Verantwortung, Schattauer Verlag, Stuttgart, 48-51

Klaschik E, Ostgathe C, Nauck F (2001): Grundlagen und Selbstverständnis der Palliativmedizin. Palliativmedizin, $\underline{2}, 71-75$

Klaschik E et al. (2002): Palliativmedizin. Defizite in der studentischen Ausbildung. Deutsches Ärzteblatt, $\underline{99}$, A1286 
Körtner U, Kopetzki C, Kletečka-Pulker M, Inthorn J (2009): Studie über die rechtlichen, ethischen und faktischen Erfahrungen nach In-Kraft-Treten des PatientenverfügungsGesetzes (PatVG), Studie im Auftrag des Bundesministeriums für Gesundheit Österreich, http://www.bmg.gv.at/cms/home/attachments/3/9/7/CH1096/CMS1261481034491/ ierm_endbericht_patvg_dez_2009.pdf, letzter Zugriff am 19.03.2016

Kowal S, O'Connell DC (2003): Zur Transkription von Gesprächen. In: Flick U, Kardoff E, Steinke I (Hrsg.): Qualitative Forschung. Rowohlt Taschenbuch Verlag, Reinbeck bei Hamburg, 437-447

Laske A et al. (2010): Palliativmedizinische Lehre in Deutschland Bestandsausfnahme an den medizinischen Fakultäten 2009, Palliativmedizin, 11, 18-25

Liebold R, Trinczek R (2009): Experteninterview. In: Kühl S et al. (Hrsg.): Handbuch Methoden der Organisationsforschung, Quantitative und qualitative Methoden. VS Verlag für Sozialwissenschaften, $32 \mathrm{ff}$.

Luhmann N (1965): Grundrechte als Institution. Ein Beitrag zur politischen Soziologie, Berlin

Lübbe AS (2014): Für ein gutes Ende. Von der Kunst, Menschen in ihrem Sterben zu begleiten. Erfahrungen auf einer Palliativstation, Heyne, München

Macklin R (2003): Dignity is a useless concept. In: British Medical Journal Journal $\underline{327}$ (7429), 1419-1420

Maio G (2008a): Was ist eine guter Arzt? - Ethische Überlegungen zur ärztlichen Identität in ihrem modernen Wandel, Praxis Schweizerische Rundschau für Medizin, 97, 11, 623-627

Maio G (2008b): Für eine andere Kultur des Sterbens. Die Patientenverfügung als Voraussetzung für ein gutes Sterben? Neue Zürcher Zeitung, No. 86, 11-12

Maio G (2013): Der Arzt als Ingenieur für den Menschen? Psychologische Medizin, 24/ 2 
Mayring P (2010): Qualitative Inhaltsanalyse, Grundlagen und Techniken, Beltz Verlag, Weinheim

Meredith C et al. (1996): Information needs of cancer patients. In West Scotland: cross sectional survey of patients view. Br Med J 313:724-726

Mill JS (1974): Über die Freiheit, Reclam Verlag, Stuttgart. (Basierend auf: Mill JS: On liberty, London, 1859)

Müller-Busch HC (2005): Patientenautonomie am Lebensende aus (palliativ-)medizinischer Sicht. BtPrax, 2, 50-52

Nauck F, Jaspers B (2011): Patientenverfügung als Vertrauensbildende Maßnahme. In:

Höver $\mathrm{G}$ et al. (Hrsg.): Sterbebegleitung: Vertrauenssache. Würzburg, Verlag Königshausen und Neumann, 175-192

Nauck F, Jaspers B (2012): Die Arztrolle in unterschiedlichen klinischen Kontexten. Bundesgesundheitsbl, $\underline{55}, 1154-116$

Nauck F et al. (2011): Organisation und Qualität der ärztlichen Versorgung in den Hospitzen in NRW vor Einführung der Spezialisierten Ambulanten Palliativversorgung (SAPV), Palliativmedizin, 12, 80-86

Nauck F et al. (2016): Selbstbestimmung braucht Vertrauen - Entscheidungen am Lebensende. In: Autonomie und Vertrauen. Schlüsselbegriffe der modernen Medizin. Springer VS, Wiesbaden, 101-132

Nida-Rümelin J (2009): Rationalität und Verantwortung. In: Kick HA und Unger F (Hrsg.) Verantwortung und Vertrauen, Mattes Verlag, Heidelberg

Nordenfelt L (2004): The Varieties of Dignity. In: Health Care Analysis 12 (2), 69-81

Novy K (2012): Autonomes Handeln. Soziologische, feministische und psychodramatische Perspektiven. Z Psychodrama Soziometr 11 (4) 
O’Neill O (2002): Autonomy and trust in bioethics, Cambrige University Press

Ong LML, de Haes JCJ et al. (1995): Doctor-Patient Communication: a review of the literature. Soc Sci Med $\underline{40}$ (7), 903-918

Parson T (1947): Introduction. In: Weber M, The Theory of Social and Economic Organization, Oxford University Press, New York

Paton HJ (1962): Der kategorische Imperativ. Eine Untersuchung über Kants Moralphilosophie, Berlin 1962

Quante M (2002): Personales Leben und menschlicher Tod. Personale Identität als Prinzip der biomedizinischen Ethik, Frankfurt a.M.

Radbruch L, Nauck F et al. (2002): What Is Palliative Care in Germany? Results from a Representative Survey. J Pain Symptom Manage 23 (6), 471-483

Radbruch L et al. (2003): What are the problems in palliative care? Results from a representative survey. Support Care Cancer 11 (7), 442-451

Rehbock T (2002): Autonomie - Fürsorge - Paternalismus. Zur Kritik (medizin)ethischer Grundbegriffe. Ethik Med 14,131-150, 145

Saake I (2003): Die Performanz des Medizinischen. Soziale Welt 54 (4), 429-459

Sabatowski R et al. (2001): Entwicklung und Stand der stationären palliativmedizinischen Einrichtungen in Deutschland. Schmerz 15, 312-9

Schaber P (2003): Menschenwürde als Recht, nicht erniedrigt zu werden. In: Stoecker R (Hrsg.) Menschenwürde - Annäherung an einen Begriff. Wien, öbv\&hpt, 119-131

Schicktanz S, Schildmann J (2009): Medizinethik und Empirie - Standortbestimmungen eines spannungsreichen Verhältnisses. Ethik in der Medizin 21 (3), 183-186 
Schildmann J et al. (2010): End-of-life practices in palliative care: a cross sectional survey of physican members of the German Society for Palliative Medicine, Palliative Medicine $\underline{24}, 820-832$

Schöne-Seifert B (2007): Grundlagen der Medizinethik. Alfred Kröner Verlag, Stuttgart, 39 $\mathrm{ff}$.

Schreiber H-L (2002): Palliativmedizin in Deutschland - nicht eingelöster gesellschaftlicher Anspruch an die moderne Medizin. In: Aulbert E et al. (Hrsg), Palliativmedizin als Ausdruck gesellschaftlicher Verantwortung, Schattauer Verlag, Stuttgart, 41-47

Steinfath H (2016): Das Wechselspiel von Autonomie und Vertrauen. In: Autonomie und Vertrauen. Schlüsselbegriffe der modernen Medizin, Springer VS, Wiesbaden, 11-68

Steinfath H, Pindur A-M (2013): Patientenautonomie im Spannungsfeld philosophischer Konzeptionen von Autonomie. In: Wiesemann C und Simon A (Hrsg.): Patientenautonomie. Theoretische Grundlagen, Praktische Anwendungen. Mentis, Münster, 27-41

Stiehl S et al. (2010): Palliativmedizin im Spiegel der Zeit - Ein Vergleich der Ergebnisse der Hospitz- und Palliativerhebungen von 2004 und 2009, Palliativmedizin, Ausgabe 11, 78-84

Stoecker R (2010): Die Pflicht dem Menschen seine Würde zu erhalten. In: Debus et al. (Hrsg.): Zeitschrift für Menschenrechte. Wochenschau Verlag, Schwalbach/Ts. Zfmr $1 / 2010$

Stoecker R (2011b): Die philosophische Schwierigkeiten mit der Menschenwürde - und wie sie sich vielleicht lösen lassen. Information Philosophie, 01/2011, 8-19

Student C, Napiwotzky A (2007): Palliative Care, Georg Thieme Verlag KG, Stuttgart

Taupitz J (2009): Verantwortung und Vertrauen aus rechtlicher Perspektive - insbesondere im Bereich des Gesundheitssystems. In: Kick HA und Unger F (Hrsg.) Verantwortung und Vertrauen, Mattes Verlag, Heidelberg 
Taupitz J (2009): Verantwortung - Verantwortungsfähigkeit. In: Kick HA und Unger F (Hrsg.) Verantwortung und Vertrauen, Mattes Verlag, Heidelberg

Temel JS et al. (2010): Early Palliative Care for Patients with Metastatic Non-Small-Cell Lung Cancer. N Engl J Med; $\underline{363}$ (8), 733-742

Toellner R (1991): Tradiertes Arztbild und ärztliche Ethik im Wandel der Zeit. Fortschritte der antimikrobiellen und antineoplastischen Chemotherapie. 10/11, 1-8

Vollmann J (2000): Einwilligungsfähigkeit als relationales Modell. Klinische Praxis und medizinethische Analyse. Der Nervenarzt, 72, 709-714

Wenrich MD et al. (2001): Communicating with dying patients within the spectrum of medical care from terminal diagnosis to death. Arch Intern Med 161 (6): 868-874

Wiesemann C (2012): Autonomie als Bezugspunkt einer universalen Medizinethik. Ethik Med $2 \underline{4}(4), 287-295$

Wiesemann C (2016): Vertrauen als moralische Praxis - Bedeutung für Medizin und Ethik. In: Autonomie und Vertrauen. Schlüsselbegriffe der modernen Medizin, Springer VS, Wiesbaden, 69-99

Wiesemann C, Simon A (2014): Patientenautonomie. Theoretische Grundlagen, Praktische Anwendungen. Mentis, Münster

Wils J-P (2002): Zentrale Begriffe der Ethik. In. Düwell M et al. (Hrsg.), Handbuch Ethik, JB Metzler Verlag, Stuttgart/ Weimar, 537-541

Wils J-P, Wolff M (1999): Ethik ohne Chance? Erkundungen im techno-logischen Zeitalter, Tübingen

Witzel A (2000): Das problemzentrierte Interview, Forum: Qualitative Sozialforschung, Vol. 1, No.1, Art. 22, URL http://www.qualitative-research.net/index.php/fqs/article/view/ 1132/2519 (letzter Zugriff am 22.09.2015) 
Woydack L (2015): Die Realisierbarkeit des Autonomieprinzips - Überlegungen zwischen den Forderungen der Öffentlichkeit und den Reflexionen der medizinischen Praxis. ZEE, Heft 3, 179-190

Woydack L, Inthorn J (2013): Das Autonomieprinzip in der Palliativmedizin in Theorie und Praxis. In: Anselm R et al. (Hrsg.) Autonomie und Macht. Interdisziplinäre Perspektiven auf medizinethische Entscheidungen, Edition Ruprecht, Göttingen

Zigmond AS, Snaith RP (1983): The Hospital Anxiety and Depression Scale. Acta Psychiatr Scand $\underline{67}$ (6), 361-370 


\section{Anhang}

Interviewleitfaden

\section{Gesprächssituation/ Rahmenbedingungen}

1. Können Sie mir aus ihrer Erfahrung heraus eine typische Gesprächssituation schildern, wie sie sich im Verlauf eines Aufklärungsgespräches für eine Krebsdiagnose o.ä. ereignet? (Setting/ wie lange kannten Sie den Patienten zu diesem Zeitpunkt?)

2. Was ist Ihnen bei einem Aufklärungsgespräch mit einem Patienten besonders wichtig? (Was soll in Ihren Augen im besten Fall nicht passieren? welche Rolle spielt die Autonomie des Patienten? wie ist es bei „nicht-autonomen Patienten?“)

3. Können Sie mir jeweils ein Beispiel für ein besonders gut und ein besonders ungünstig verlaufenes Gespräch nennen? Hatten Sie den Eindruck, dass das geschilderte Gespräch sowohl für Sie als auch für den Patienten gut verlaufen ist?)

4. Wie viel wollen Ihre Patienten selbst bestimmen? Was delegieren Patienten eher an Sie? (wie wird Ihnen dieser Aspekt kommuniziert?)

\section{Rhetorische Stilmittel}

1. Wodurch ist für Sie ein gutes Arzt-Patienten-Gespräch gekennzeichnet? (was genau ist Ihnen dabei wichtig? Motive: was ist Ihr Ziel dabei?)

2. Wenn Sie sich an Situationen erinnern, in denen es um die Gestaltung des Lebensendes ging... in welcher Form wollen Patienten mit Ihnen über den Tod sprechen? Was wird nicht angesprochen oder auffällig oft ausgeklammert? Besteht auf Ihrer Seite manchmal Wut über eine fehlende Bereitschaft zu Kommunikation seitens der Patienten?

3. Wie genau kommunizieren Sie Ihren Patienten (Ihre) Ziele in der palliativen Situation? (Stichwort „Gestaltung des Lebensendes, „Mitentscheiden“, „Schmerzfreiheit“...)

4. Welche Elemente bezüglich der Gestaltung des Lebensendes sind Ihnen wichtig? (Was verknüpfen Sie mit den Begriffen Lebensqualität, würdevolles Sterben, Autonomie am Lebensende?)

5. Es herrscht eine Art Konsens darüber, dass man den bevorstehenden (würdevollen) Tod „gestalten kann“ oder sogar „gestalten soll“... Was ist Ihnen diesbezüglich wichtig? Was sollte ein Patient in der Sterbesituation haben?

6. In welcher Form versuchen Sie herauszufinden, was einzelne PatientInnen beispielsweise unter dem Aspekt „Lebensqualität“ verstehen?

III. Sonstiges 
1. Alter und Geschlecht des Interviewpartners

2. Institution, Station, medizinischer Fachbereich, Jahre im Beruf, Bezug zur Palliativmedizin 
15. Publikationsliste

Teile dieser Dissertation wurden bereits publiziert:

Woydack L (2015): Die Realisierbarkeit des Autonomieprinzips - Überlegungen zwischen den Forderungen der Öffentlichkeit und den Reflexionen der medizinischen Praxis. ZEE, Heft 3, 179-190

Woydack L, Inthorn J (2013): Das Autonomieprinzip in der Palliativmedizin in Theorie und Praxis. In: Anselm R et al. (Hrsg.) Autonomie und Macht. Interdisziplinäre Perspektiven auf medizinethische Entscheidungen, Edition Ruprecht, Göttingen 


\section{Danksagung}

Besonders danken möchte ich hiermit meiner Betreuerin Frau Professor Dr. Silke Schicktanz sowie den KollegInnen des Instituts für Ethik und Geschichte der Medizin, die mich inspiriert haben, eine Dissertation im medizinethischen Bereich zu schreiben und darüber hinaus mein gesamtes Studium sehr bereichert haben. Die universitären Kurse, die Kolloquien und die Doktorandenworkshops haben mich nicht nur fachlich, sondern auch zwischenmenschlich sehr bereichert. Darüber hinaus prägen sämtliche medizinethische Erfahrungen, die ich über das Team des Instituts für Ethik und Geschichte in der Medizin sowie durch Fachtagungen gesammelt habe, meine ärztliche Tätigkeit maßgeblich.

Bedanken möchte ich mich auch für die stets hervorragende Betreuung durch Frau Professor Dr. Silke Schicktanz und ihr Team. Dabei gebührt ein ganz besonderer Dank Frau Dr. Julia Inthorn, die mich mit zahlreichen fachlichen Diskussionen, Inspirationen und Korrekturarbeiten unterstützt hat. 